\title{
Survey of flight and numerical data of hypersonic rarefied flows encountered in Earth orbit and atmospheric reentry
}

\author{
Marc Schouler ${ }^{\mathrm{a}, *}$, Ysolde Prévereaud ${ }^{\mathrm{a}}$, Luc Mieussens ${ }^{\mathrm{b}}$ \\ ${ }^{a}$ ONERA / DMPE, Université de Toulouse, F-31055 Toulouse, France \\ ${ }^{b}$ Bordeaux INP, Univ. Bordeaux, CNRS, IMB, UMR 5251, F-33400 Talence, France
}

\begin{abstract}
The control of the satellites end-of-life including deorbiting and atmospheric entry as well as the investigation of orbital maneuvers for new space missions confer a growing importance to the study of the hypersonic rarefied regime. While flight data are necessary for the validation of Direct Simulation Monte-Carlo numerical simulations, only a few studies and data are available for this purpose. Therefore, this article aims at gathering ground windtunnel and flight data in rarefied regime as well as their numerical reconstruction from a wide scope of space programs. A detailed analysis of these data will be presented. After a review of hypersonic low-density wind-tunnel experiments that are the main source of non-reacting hypersonic rarefied flow study cases, we address, with our own simulations, unexploited afterbody heating data from former space programs such as Mercury and Apollo. Numerically reconstructed flight data from OREX and the Space Shuttle are also considered. Finally, ionization and radiative environment are discussed through the data collected during Stardust, Fire II and RAM-C II Earth atmospheric entry.
\end{abstract}

Keywords: Direct Simulation Monte-Carlo, Rarefied hypersonic flow, Earth reentry, Experimental and flight data

\section{Contents}

1 Introduction $\quad 2$

2 Low density wind tunnel experiments $\quad 3$

2.1 Rarefied hypersonic flow over a flat plate . . . . . . . . . . . . . 3

2.2 Rarefied hypersonic flow over a flat plate with a sharp leading edge . . . . . 4

2.3 Rarefied hypersonic flow over a $70^{\circ}$ blunted cone . . . . . . . . . . . . 6

3 Aerothermodynamic flight data $\quad 10$

3.1 The Mercury Project . . . . . . . . . . . . . . . . . . . . 10

3.2 The Apollo Program . . . . . . . . . . . . . . . . . . . . . . . . . . . 14

3.2.1 Apollo 6: Aerodynamic coefficients simulation . . . . . . . . . . 15

3.2.2 Apollo AS-202: Afterbody heat flux simulation . . . . . . . . . . . 19

3.3 The Space Shuttle Orbiter . . . . . . . . . . . . . . . . . . . 25

3.4 The Orbital Reentry Experiment - OREX . . . . . . . . . . . . . 28

3.5 MIRKA . . . . . . . . . . . . . . . . . . . . . . 30

* Corresponding author

Email address: marc.schouler@onera.fr (Marc Schouler) 
4.1 Fire II . . . . . . . . . . . . . . . . . . . . . . . . . . . . . . . 32

4.2 RAM C-II . . . . . . . . . . . . . . . . . . . . . . . . . . 35

4.3 The sample return capsule Stardust . . . . . . . . . . . . . . . . . 36

\section{Conclusion}

\section{Introduction}

A good knowledge of rarefied hypersonic flows is critical for the study of the feasibility of satellite orbital transfer maneuvers from low to very low orbit, as well as for an accurate prediction of reentry trajectory of both space debris and spacecraft. Indeed, an accurate computation of the aerodynamic coefficients of a flying object in high atmosphere is crucial for a precise prediction of its trajectory and its stability. In the same way, a precise estimation of the heat flux applied to satellites or spacecraft is required for a proper design of their thermal protection system (TPS) and for the risk management of debris demise.

Usually the degree of rarefaction of a gas is quantified by the Knudsen number $K n=\lambda / L$ where $\lambda$ is the gas mean free path $(\mathrm{m})$ and $L$ is the characteristic length of the object in the flow $(\mathrm{m})$. The rarefied regime can then be divided into three sub-regimes. When $K n>10$, the flow is free molecular which means that inter-molecular collisions can be neglected and the gas only interacts with the object's walls. The regime is said to be transitional for $0.1<K n<10$. In this regime, inter-molecular collisions effects start to be significant but not enough to reach a local equilibrium. When $0.001<K n<0.1$, the gas is in a slip flow regime and transitional non-equilibrium is important near surfaces only. Finally, the flow is considered to be in a continuum regime when $K n<0.001$. During the first phase of atmospheric reentry, the object goes through all these regimes. Before the atmospheric layers become dense enough for the flow to be in a continuum regime, a non negligible deviation from the thermochemical equilibrium leads to the failure of the classical NavierStokes conservation equations and particle simulation methods must be used.

In this context, the Direct Simulation Monte-Carlo (DSMC) method introduced by Bird [1] has proven to be one of the most appropriate numerical approaches for the simulation of rarefied flows (from near-continuum to free-molecular). This method consists of an algorithm that solves the Boltzmann equation and which computes the outcome of particle collisions through stochastic processes. Over the past decades, the method has continually increased its capacities of simulating thermochemical non-equilibrium phenomena which are involved in atmospheric reentry conditions [2]. The severe conditions found in such flows are hardly reproducible in ground facilities, which is why flight data are particularly valuable for the validation of DSMC.

Several authors have addressed the issue of atmospheric entry and the gathering of flight data but none of these were dedicated to the rarefied regime encountered during Earth atmospheric reentry. Wright et al. [3] investigated afterbody aeroheating flight data in continuum regime and Reynier [4] gathered aerothermodynamics flight data in the frame of Mars exploration projects. Hollis and Borrelli [5] studied the aerothermodynamics of blunt body entry vehicles. A specific part of this work discusses the rarefied regime for which numerical heat fluxes computed with DSMC and CFD are compared for a Mars Science Laboratory like vehicle between 85 and $95 \mathrm{~km}$. Finally, Schwartzentruber and Boyd, in their paper about progress of particle-based simulation of hypersonic flows [6], presented data from various experiments and flights. The double cones and cylinder-flares experiments in the LENS facility (pressure and heat flux) as well as on-board measurements of the BowShock Ultra-Violet-2 (ultra-violet emission) and the RAM-C II (electron number density) flights are discussed. 
In this paper, the focus is given on experimental data obtained in ground facilities that are commonly used for DSMC benchmark and validation purposes, on inferred data and on flight data measured during Earth reentry. Some of these data have already been used and published in a numerical validation context. Some data were processed and used here for the first time and some are given and analyzed but are yet to be fully exploited.

\section{Low density wind tunnel experiments}

Rarefied atmospheric reentry flows are characterized by low densities, hypersonic velocities and high enthalpy. High-enthalpy shock facilities have been reviewed by Reynier [7] in 2016. The only facilities able to reproduce reentry conditions are shock-tubes, shock-tunnels, expansion tubes and hot-shots. However, these facilities only work for short duration and their densities do not match those necessary to retrieve rarefied conditions. Hypersonic, high-enthalpy and low density flow synthesis is a technological challenge that is still under consideration. Hence, the only low density wind tunnel results available are for non-reactive flows.

\subsection{Rarefied hypersonic flow over a flat plate}

Hypersonic rarefied flows over flat plates have been largely studied both experimentally and numerically. The simplicity of the geometry and the experimental results precision makes this case particularly useful for numerical validation. In this section, we focus on the study of the rarefied hypersonic flow over a flat plate with a truncated leading edge.

Initially, this experiment was conducted by Allegre et al. [8] in the SR3 wind tunnel of Centre National de la Recherche Scientifique (CNRS) Meudon. A pure nitrogen $\left(\mathrm{N}_{2}\right)$ flow was injected with two freestream conditions: $M_{\infty}=20.2, R e_{\infty}=2850$ and $R e_{\infty}=8380$ at a temperature $T_{\infty}=13.32 \mathrm{~K}$. The density flowfields were monitored by electron beam surveys and the wall pressure and convective heat flux were measured through pressure orifices and a thin skin technique. The plate was $100 \mathrm{~mm}$ long $\left(L_{p}\right), 100 \mathrm{~mm}$ wide, $5 \mathrm{~mm}$ thick and the wall temperature was maintained at $290 \mathrm{~K}$. In this experiment, two angles of attack $\left(0^{\circ}\right.$ and $10^{\circ}$ ) were investigated.

The results of this work were widely used afterwards for the benchmarking and the validation of DSMC codes implementation [9], [10], [11]. In this context, the purpose is to simulate the hypersonic flow over the flat plate in the first freestream conditions and without incidence. We performed our own numerical simulations with the SPARTA DSMC code [12]. A total of around 4 million particles were simulated in a $2 \mathrm{D}$ domain $\left[x_{\min } ; x_{\max }\right] \times$ $\left[y_{\min } ; y_{\max }\right]=[-0.06 ; 0.12] \times[-0.1025 ; 0.1025]$ with dimensions in $\mathrm{m}$. A $360 \times 410$ grid was used and Allegre's first freestream conditions were applied. By using the variable hard sphere (VHS) collision model, the mean free path becomes:

$$
\lambda_{\infty}^{\mathrm{VHS}}=\frac{1}{\sqrt{2} \pi d_{\mathrm{ref}}^{2} n_{\infty}}\left(\frac{T_{\infty}}{T_{\mathrm{ref}}}\right)^{\omega-1 / 2} .
$$

With a numerical density $n_{\infty}=3.716 \times 10^{20} \cdot / \mathrm{m}^{3}$, a molecular diameter $d_{\text {ref }}=4.17 \times 10^{-10}$ $\mathrm{m}$ and a viscosity index $\omega=0.74$ at reference temperature $T_{\text {ref }}=273 \mathrm{~K}$, this ultimately results in a mean free path $\lambda_{\infty}=1.6 \mathrm{~mm}$ and a Knudsen number $K n=0.016$. The velocity can be computed from the Mach number which gives $U_{\infty}=1503 \mathrm{~m} / \mathrm{s}$. Similarly to Padilla's recommendations [9], energy exchange between the translational and rotational mode was allowed and performed with the Larsen-Borgnakke model [13] with a constant rotational number $Z_{\text {rot }}=5$. The complementary numerical parameters (time step, sampling parameters, number of run, etc) were taken in accordance with those indicated in his paper. 
Figures $1 \mathrm{a}$ and $1 \mathrm{~b}$ show the evolution of the pressure and heat flux along the upper surface of the plate. Similarly to what was suggested by Allegre, two simulations were realized with different gas-surface interaction conditions. For the first simulation, diffuse reflection is used with full thermal accommodation $(w=1)$ while the second simulation uses an accommodation coefficient $w=0.8$. This value corresponds to literature prescriptions for a nitrogen flow over a steel plate at temperature $T_{w}=300 \mathrm{~K}$ [14], [15]. With $w=1$ and for both quantities, Padilla's results [9] obtained with the DSMC codes DAC [16], and MONACO [17] show a very good agreement with our results obtained with SPARTA. However, Figure 1a displays significant differences between the experimental and numerical heat flux. The small change in the accommodation coefficients $(w=0.8)$ leads to a clear improvement of both the pressure and the heat flux and an excellent agreement is reached between SPARTA and the experimental results.

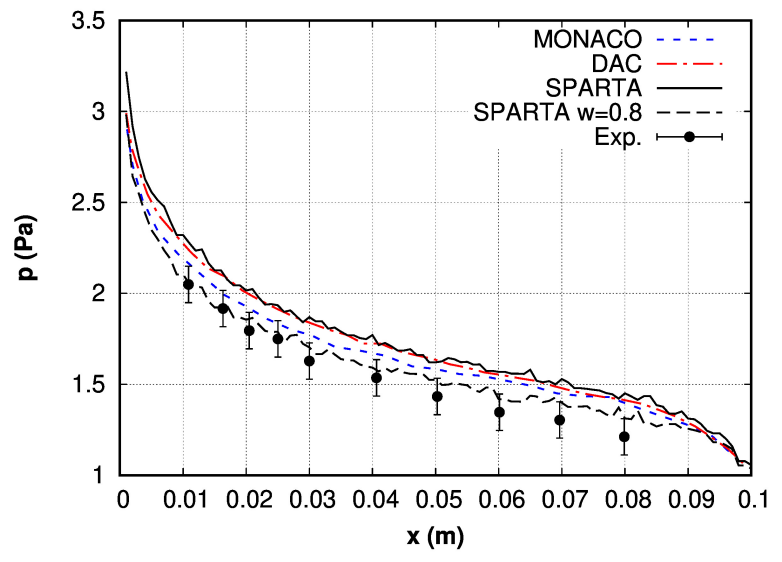

(a) Wall pressure distribution

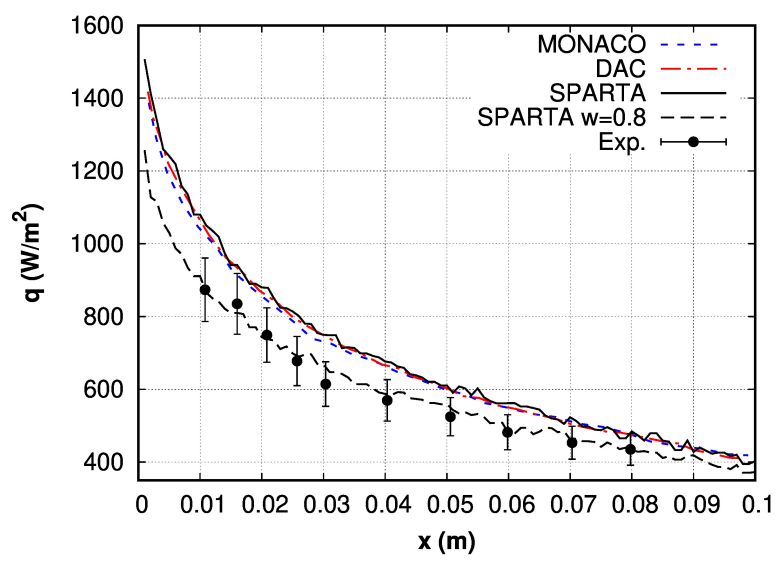

(b) Wall heat flux distribution

Figure 1: Comparison of the wall pressure and heat flux distributions over a flat plane obtained from DSMC simulations and experimental tests at $M_{\infty}=20.2, K n=0.016$ and $\alpha=0^{\circ}$.

\subsection{Rarefied hypersonic flow over a flat plate with a sharp leading edge}

The investigation of the effect of sharp leading edge angles on pressure and heat flux distribution along the flat plate was conducted by Heffner et al. [18] and Lengrand et al. [19]. Bevel angles variation between 0 and $80^{\circ}$ were tested by Heffner while Lengrand kept a bevel angle of $20^{\circ}$ but tested two angles of attack of respectively 0 and $10^{\circ}$. In this section, the focus is given to Lengrand's experiments conducted in the SR3 low density facility. The length of the flat illustrated in Figure 2 was $L_{p}=0.1 \mathrm{~m}$ and the tests were realized in similar conditions as those of the truncated flat plate presented in the previous section: $U_{\infty}=1503$ $\mathrm{m} / \mathrm{s}, n_{\infty}=3.716 \times 10^{20} \cdot / \mathrm{m}^{3}, T_{\infty}=13.32 \mathrm{~K}, T_{w}=290 \mathrm{~K}$ and $\alpha=0^{\circ}$. The same quantities were monitored. Besides the experimental results, Lengrand also presented numerical results obtained with a CFD code using velocity slip and temperature jump boundary conditions and results obtained with a DSMC code.

Many authors have simulated Lengrand's experiment [11], [20], [21] with several DSMC codes. They all obtained a good agreement with the experimental results but only Palharini's results obtained with the open source DSMC code dsmcFoam will be discussed in details herein. In the Benchmark of non-reacting gas flows using dsmcFoam, Palharin et al. [11] realized $3 \mathrm{D}$ simulations of the sharp plate experiment with a bevel angle of $20^{\circ}$ and a $0^{\circ}$ angle of attack. In the computations, 13 numerical particles per cell were modeled and a grid of 4.7 million cells was employed. The domain dimensions and all the numerical parameters are specified in the paper [11]. 


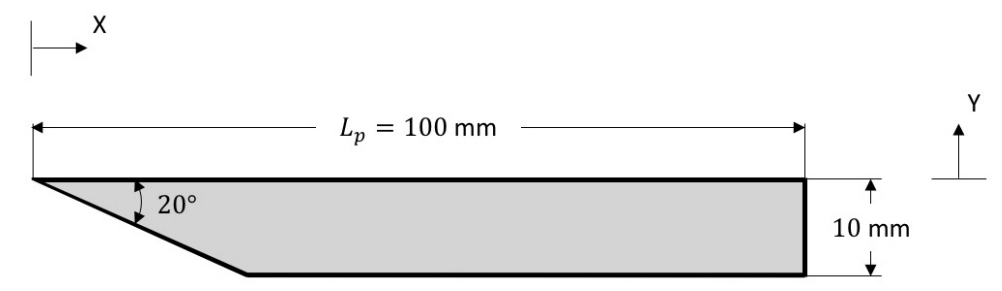

Figure 2: 2D schematic of the sharp plate and its coordinate system.

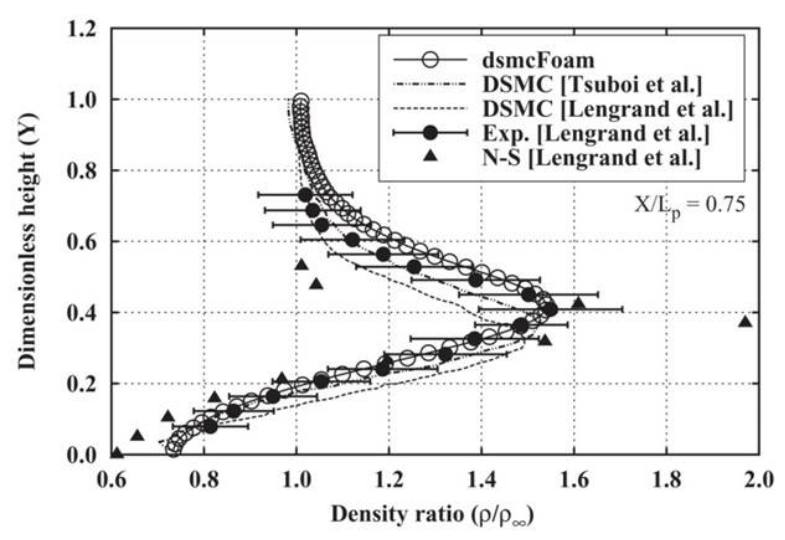

(a) Normalized density profile

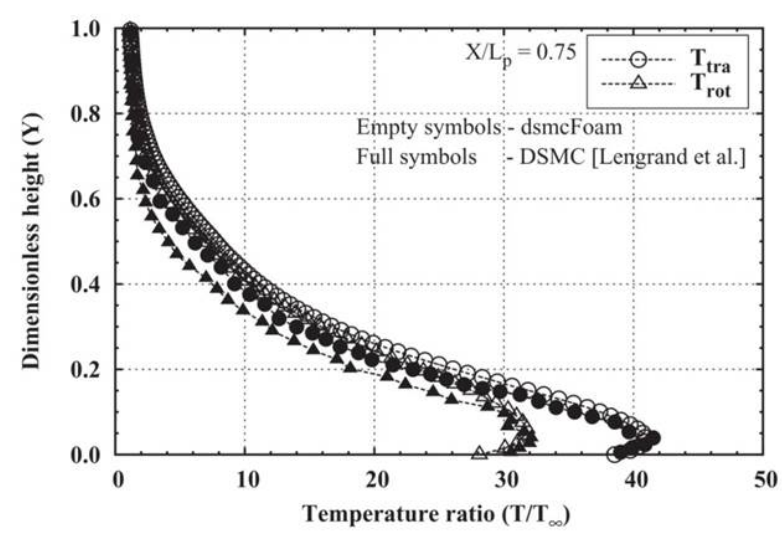

(b) Normalized temperature profile

Figure 3: Experimental versus numerical results normal to the plate at streamwise position $X / L_{p}=0.75$ (from [11]).

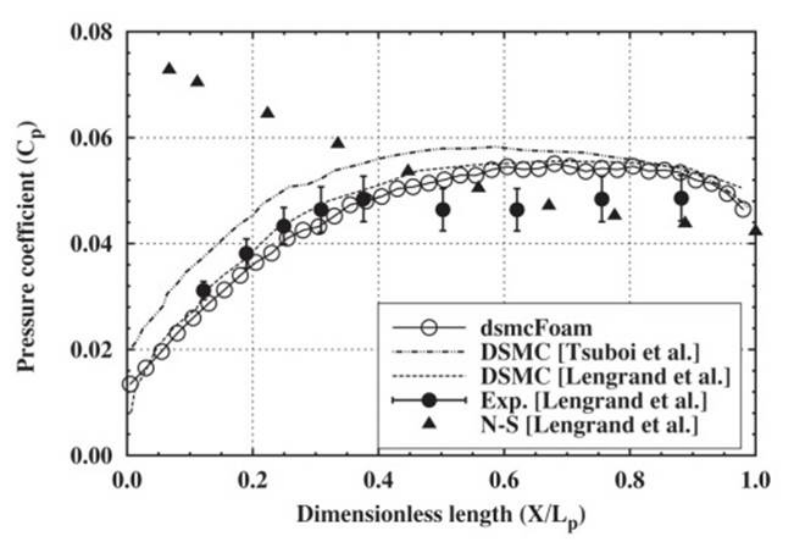

(a) Pressure coefficient

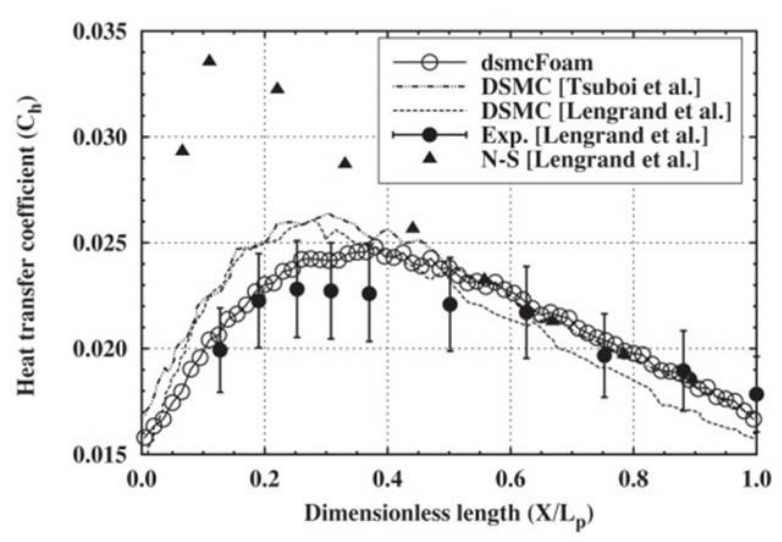

(b) Heat transfer coefficient

Figure 4: Experimental versus numerical surface coefficients along the plate (from [11]).

The profiles of normalized density $\left(\rho / \rho_{\infty}\right)$ and normalized temperature $\left(T / T_{\infty}\right)$ normal to the plate at the position $X / L_{p}=0.75$ are given in Figures $3 \mathrm{a}$ and $3 \mathrm{~b}$. An excellent agreement is shown between the dsmcFoam and the experimental results for the density. Figure 3a also illustrates the incapacity of the CFD simulation to adequately capture the density profile at that position. Figure $3 \mathrm{~b}$ shows the thermal non-equilibrium conditions with the difference of translational and rotational temperature that is captured by both DSMC codes. Figure $4 \mathrm{a}$ and $4 \mathrm{~b}$ show the evolution of the pressure $\left(C_{p}\right)$ and heat transfer $\left(C_{h}\right)$ coefficients along the plate. Overall, a good agreement is observed between the DSMC results and the experimental data but a slight over-estimation of $C_{p}$ is given by the DSMC computations. Furthermore, the Navier-Stokes (N-S) simulations are unable to correctly compute the two surface coefficients when $X / L_{p}<0.4$. According to Lengrand et al. [19], better results are obtained if the velocity slip and temperature jump are not too large. In 
the conditions of study, this is not the case for the region $X / L_{p}<0.4$. The nonequilibrium is too significant and cannot be properly modelled with such continuum approach.

Although this experiment is mostly used for validation purposes, such experiments have a larger scope of application. Gas-surface interaction models were assessed by Padilla [22], [23] with a similar flat plate experiment condutcted by Cecil and McDaniel [24]. In this work, the Boundary-layer profiles and surface-property distributions which were measured by the experiment are compared with DSMC results obtained with Maxwell and Cercignani, Lampis and Lord (CLL) models. Results showed that both models lead to similar boundarylayer profiles and aerodynamic results for gas-surface accommodation between 50 and 100\%. Moreover, a $90 \%$ gas-surface accommodation was found to produce the best agreement with the velocity measurements but as stated by Padilla, additional study is required to assess the models ability to retrieve heat transfer measurements.

\subsection{Rarefied hypersonic flow over a $70^{\circ}$ blunted cone}

The AGARD working group and more specifically Moss and Lengrand [25] made a review of the experimental and numerical efforts carried out for the Mars Pathfinder which is a $70^{\circ}$ blunted cone shaped probe. This report gathers data from experiments conducted in six facilities: the SR3 wind-tunnel in Meudon, the V2G-V3G-HEG of DLR-Göttingen and the LENS wind-tunnel at the University of Buffalo. Considering the experimental test conditions of each facility, the focus is given to Allegre et al. experiments conducted in the SR3 windtunnel [26], [27], [28]. The experimental conditions are given in Table 1. Three freestream conditions were generated in order to produce different levels of rarefaction and a probe model presented in Figure 5 was used.

\begin{tabular}{cccccccccc}
\hline \hline Case & $T_{0}(\mathrm{~K})$ & $P_{0}(\mathrm{bar})$ & $M_{\infty}$ & $R e_{\infty} / \mathrm{cm}$ & $\rho_{\infty}\left(\mathrm{kg} / \mathrm{m}^{3}\right)$ & $U_{\infty}(\mathrm{m} / \mathrm{s})$ & $T_{\infty}(\mathrm{K})$ & $\lambda_{\infty}(\mathrm{mm})$ & Gas \\
\hline 1 & 1100 & 3.5 & 20.2 & 284 & $1.73 \times 10^{-5}$ & 1503 & 13.3 & 0.671 & $\mathrm{~N}_{2}$ \\
2 & 1100 & 10 & 20 & 835 & $5.19 \times 10^{-5}$ & 1502 & 13.6 & 0.226 & $\mathrm{~N}_{2}$ \\
3 & 1300 & 120 & 20.5 & 7235 & $46.62 \times 10^{-5}$ & 1634 & 15.3 & 0.027 & $\mathrm{~N}_{2}$ \\
\hline \hline
\end{tabular}

Table 1: Experimental test conditions of the SV3 wind-tunnel.

Similarly to what was done with the flat plate experiments, density measurements were conducted with an electron beam and the wall temperature was kept around $290 \mathrm{~K}$. For the aerodynamic forces measurements, an aluminum probe was used with a wall temperature estimated around $350 \mathrm{~K}$. Heat transfer measurements were made with a thin-wall technique on a steel probe whose wall temperature was kept at $300 \mathrm{~K}$.

We performed several DSMC simulations with the DSMC code SPARTA for the freestream conditions corresponding to the first case. The reference length $\left(L_{\text {ref }}\right)$ is equal to the cone basis diameter which results in a Knudsen number $K n=0.013$. Once again, the flow is not energetic enough to trigger the vibrational energy mode therefore, energy exchange between the translational and rotational mode only is activated and controlled by the Larsen-Borgnakke algorithm [13]. The VHS collision model is used with a constant rotational number $Z_{\text {rot }}=5$. Two sets of simulations were completed with a varying angle of attack $\alpha$ ranging from 0 to $30^{\circ}$ and for each, the probe nose is placed at the origin of the simulation domain. For the first set, density contours and the heat transfer coefficient were computed with a wall temperature $T_{w}=290 \mathrm{~K}$. For the case without incidence, an axi-symmetric simulation was made in a $\left[x_{\min } ; x_{\max }\right] \times\left[y_{\min } ; y_{\max }\right]=[-25 ; 125] \times[0 ; 90] \mathrm{mm}$ domain with a 750 by 450 mesh and a time step $\Delta t=1.3 \times 10^{-7} \mathrm{~s}$ as prescribed by Klothakis and Nikolos [29]. For the cases with incidence, a $\left[x_{\min } ; x_{\max }\right] \times\left[y_{\min } ; y_{\max }\right] \times\left[z_{\min } ; z_{\max }\right]=[-20 ; 80] \times[-80 ; 80] \times[0 ; 80] \mathrm{mm}$ domain was used with a symmetric boundary condition on the $(x, y)$ plan. A uniform grid of $250 \times 250 \times 100$ cells was used and the adaptive mesh refinement algorithm of SPARTA was 


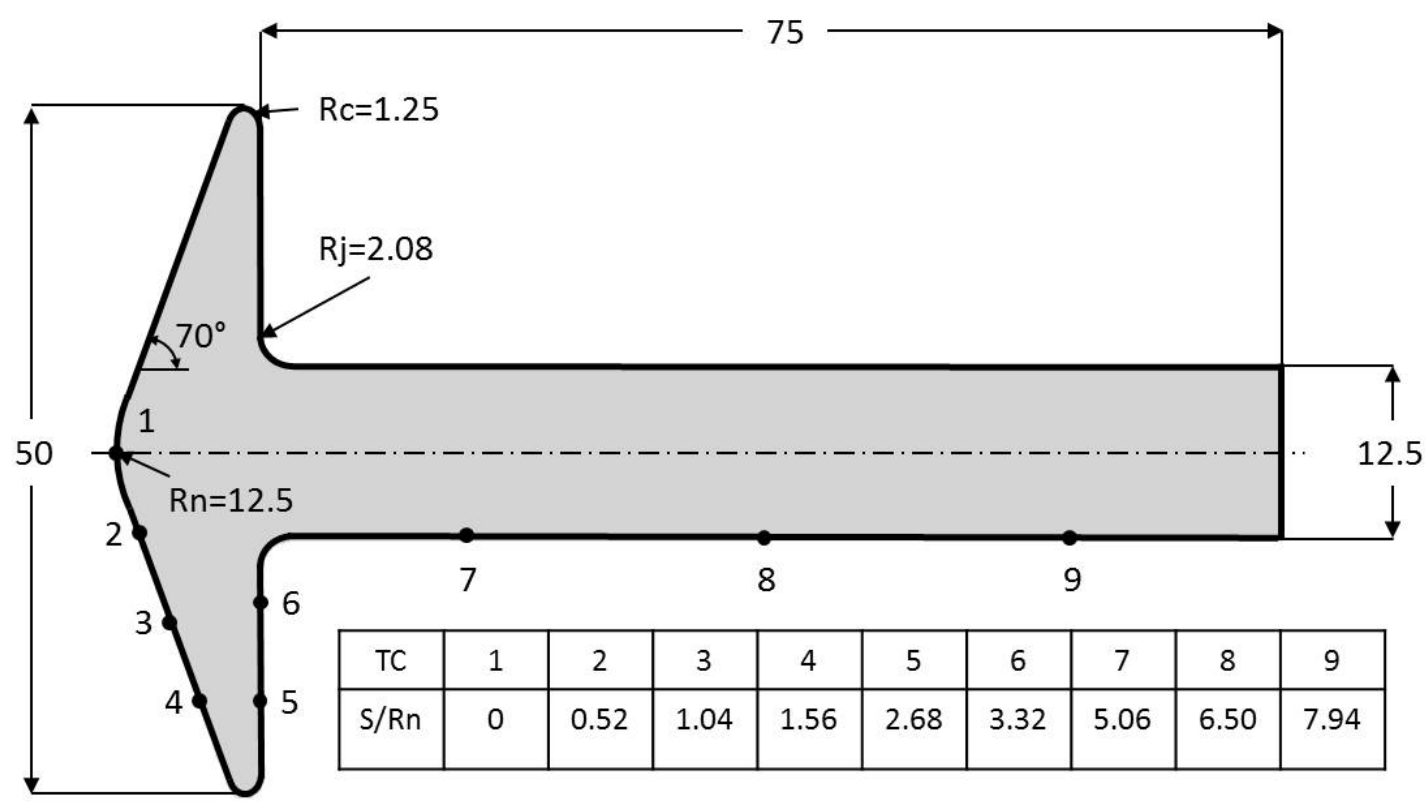

Figure 5: Experimental probe instrumentation (Thermocouples) along the curvilinear abscissa $S$ normalized by the nose radius $R_{n}$ (adapted from [27]). Dimensions in $\mathrm{mm}$.

triggered in order to insure a cell size at least two times smaller than the local mean free path in the simulation domain. For the second set of simulations, the aerodynamics forces were computed with a wall temperature of $350 \mathrm{~K}$. The domain was kept the same for all angles of attack. Thus, a $\left[x_{\min } ; x_{\max }\right] \times\left[y_{\min } ; y_{\max }\right] \times\left[z_{\min } ; z_{\max }\right]=[-20 ; 125] \times[-80 ; 80] \times[0 ; 80] \mathrm{mm}$ domain was used with a $360 \times 250 \times 100$ initial grid and the same adaptive mesh refinement algorithm. The aerodynamic forces were extracted from the nose up to $75 \mathrm{~mm}$ along the sting, according to the experimental conditions (Figure 5). For the axisymmetric simulation, the number of numerical particles was around 800 millions and for all the other simulations, this number was kept around one billion particles in order to ensure the presence of more than 10 numerical particles per cell almost all over the domain.

Surface quantities were compared with Allegre's experimental and Palharini's numerical data [11]. Figures $6 \mathrm{a}, 6 \mathrm{~b}$ and $6 \mathrm{c}$ present respectively the aerodynamic coefficients of axial $\left(C_{A}\right)$ and normal $\left(C_{N}\right)$ forces as well as the pitching moment $\left(C_{m}\right)$ coefficient whose moment reference point corresponds to the probe nose.

According to Allegre, the uncertainty on the measurement of the aerodynamic coefficients is smaller than $\pm 3 \%$. An excellent agreement is visible between the DSMC and experimental results for the axial force coefficient $\left(C_{A}\right)$ with DSMC values in the interval of uncertainty. For the normal force coefficient $\left(C_{N}\right)$ the agreement is good overall but a non-negligible deviation of $13 \%$ with SPARTA and a $8 \%$ with dsmcFoam is shown for $\alpha=30^{\circ}$. For both coefficients, the discrepancy increases with the angle of attack which inevitably impacts the pitching moment coefficient for which the numerical values deviation from the experimental results also increases with the angle of attack.

Figure 7 shows the heat transfer coefficient $\left(C_{h}\right)$ for various angles of attack. The increase in $\alpha$ leads to the shifting of the stagnation point along the spherical part of the probe. Thus, on the lower surface, the stagnation point becomes closer to the attachment point of the sonic lines on the probe shoulder (Figure 8). Furthermore, the decrease of the boundary layer thickness near the shoulder induces an increase of the heating peak in this region. At the conical trailing edge, the absence of sensor does not permit to experimentally retrieve the heat flux peak that is numerically predicted for the four angles of attack. Nonetheless, the discrepancy between the DSMC and experimental results is significant for the closest sensor 


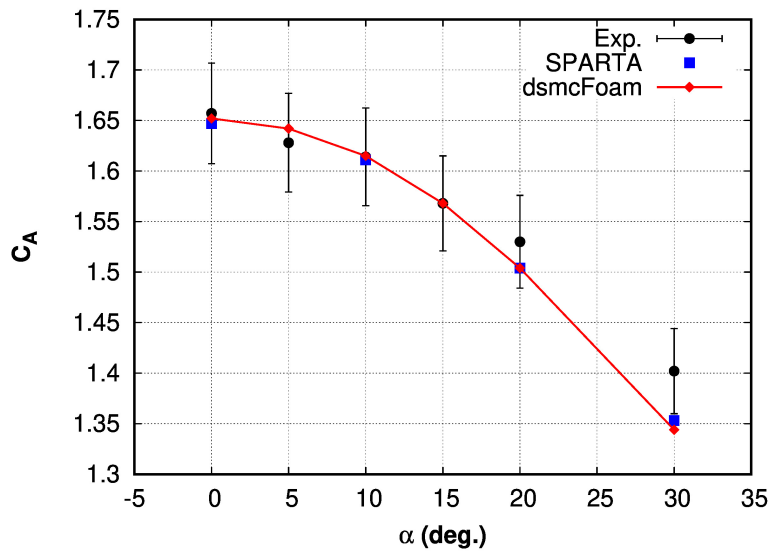

(a) Axial force coefficient

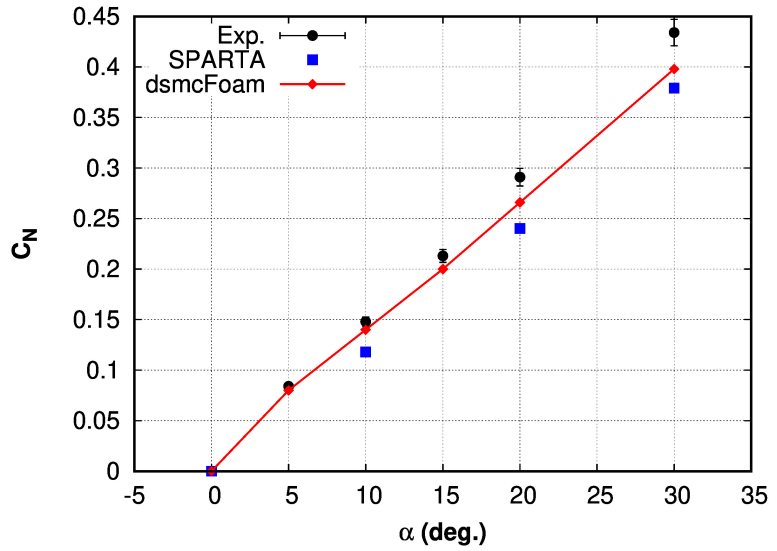

(b) Normal force coefficient

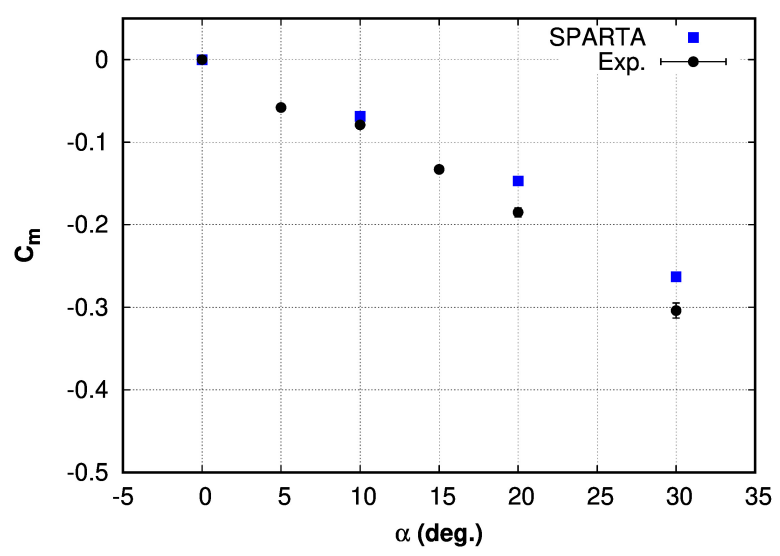

(c) Pitching moment coefficient

Figure 6: Comparison of the experimental and DSMC aerodynamic coefficients for various angles of attack.

to the trailing edge with differences from $6 \%$ to $24 \%$ according to the angle of attack. The wall heat flux coefficient obtained by DSMC in the forebody region is in good accordance with the experimental results for the 0 and 10 degrees angles of attack with mean discrepancies within $\pm 10 \%$, the range of estimated uncertainty [28]. However, the results in the forebody region show significant differences in the heat transfer coefficient of around 20 and 30\% respectively for the 20 and 30 degree angles of attack. These differences can be due to the simulation, the experiment or both. Moreover, because of the boundary layer separation near the shoulder, the flow rapidly expands and the flow trapped under the shear layer forms a recirculation zone immediately behind the probe (Figure 8). In DSMC, ensuring a sufficient number of numerical particles to reduce the statistical noise in such area requires to simulate a great amount of particles which can be computationally expensive. From an experimental perspective, the instrumentation is likely to reach its sensitivity limit in such conditions. Consequently, the complexity of the flow in the wake region might not be adequately captured numerically and experimentally [11]. The experimental uncertainty is much higher in this region than in the front area of the probe. Finally, the differences between the DSMC results come from the different mesh refinement and particle numbers that were used. The SPARTA results show the capacity of DSMC to retrieve numerical measurements in the wake closure region if a sufficient number of numerical particles is simulated but significant discrepancies are still observed in the recirculation zone.

The double cones and cylinder flares [30], [31], [32] are two other common experiments that were mentioned in the introduction. The results are not discussed here because like for the other experiments, the numerical and experimental values are in very good agreement in 


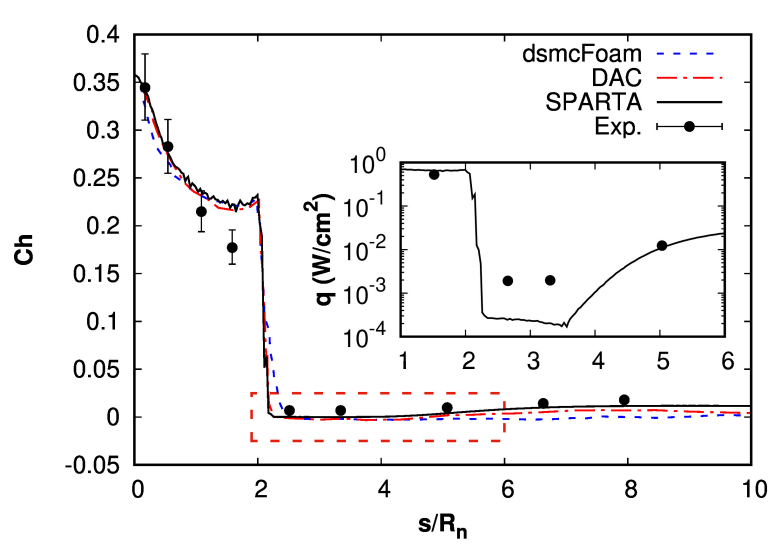

(a) $\alpha=0^{\circ}$

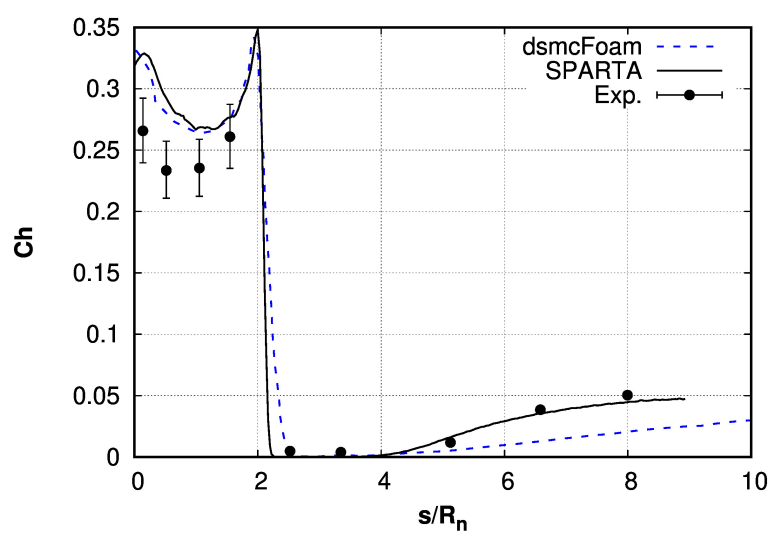

(c) $\alpha=20^{\circ}$

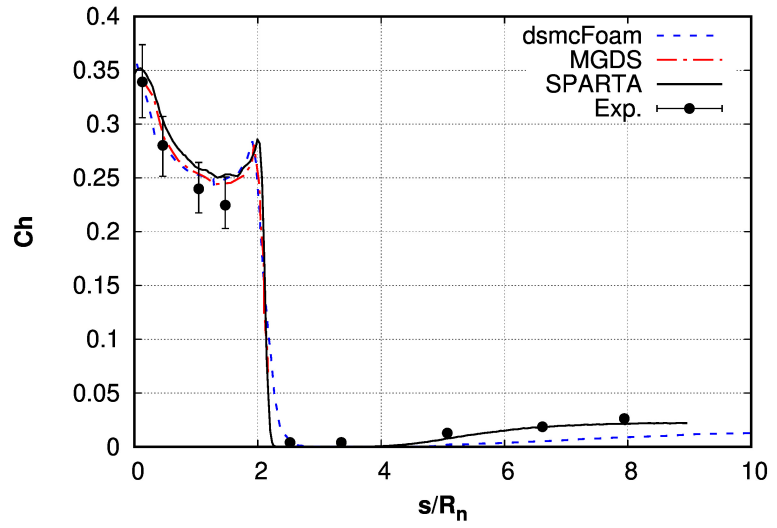

(b) $\alpha=10^{\circ}$

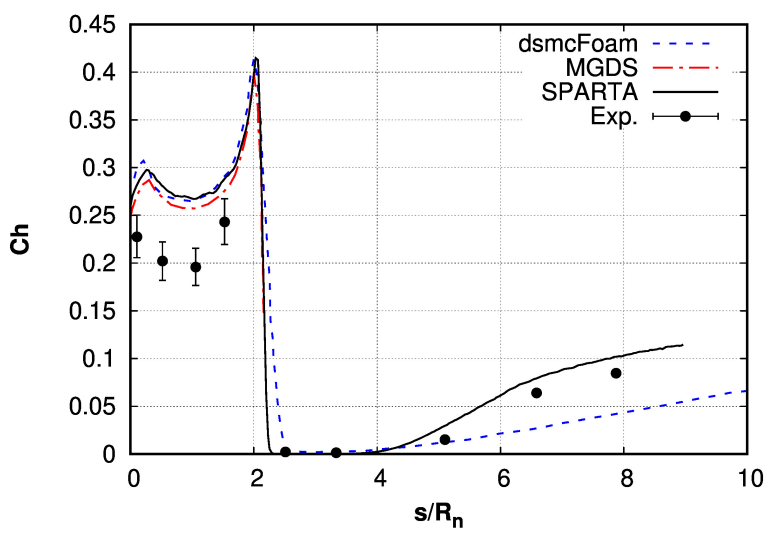

(d) $\alpha=30^{\circ}$

Figure 7: Comparison of DSMC and experimental heat transfer coefficients $(\mathrm{Ch})$ for different angles of attack.

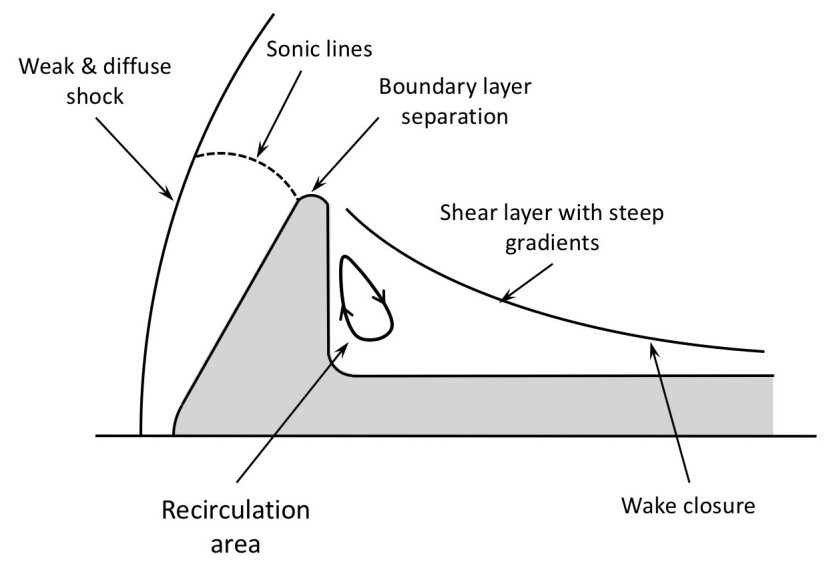

Figure 8: Illustration of the flow structure around the sphere-cone (adapted from [11]).

general and the flow is not energetic enough to trigger the vibrational mode nor any reactive process.

To summarize the results of this part, many experiments were conducted in low-density facilities and were used for numerical comparison and validation. Experiments on flat plates have been addressed as well as experiment on a $70^{\circ}$ blunted cone. For the sharp leading edge flat plate, significant differences between DSMC and CFD results have also been observed and showed the difficulty of CFD codes to retrieve experimental flowfield and surface quantities even with slip and jump boundary conditions. The $70^{\circ}$ blunted cone brings to 
play complex mechanisms and the heat transfer coefficient in the front area of the cone has proved to be hard to reproduce for angles of attack greater than $10^{\circ}$. This indicates that even in the absence of vibrational and reactive effects, the reproduction of surface quantities can be a challenging task and elementary DSMC models such as Gas-Surface interaction and translational-rotational energy exchange models call for improvements.

\section{Aerothermodynamic flight data}

The space race started with the cold war was a turning point for space exploration. From the first human spaceflight program Mercury started in 1958 up to the successful landing of the first humans on the moon in 1969, a lot of effort were carried out for the prediction of aerothermodynamic (ATD) coefficients. Although the most severe heat loads occur at low altitudes (between 40 and $60 \mathrm{~km}$ ), depending on the entry velocity and the vehicle's size, non negligible heat fluxes can be observed in the upper layers of the atmosphere. Since the entry point often coincides with an altitude close to $120 \mathrm{~km}$, on-board measurement devices sometimes provide high altitude values that can be compared to DSMC simulations. The focus of this part is given to the study and exploitation of such ATD flight data.

On ground, afterbody aeroheating is particularly challenging to evaluate. Indeed, test facilities usually use stings to maintain geometries position inside the wind-tunnel which can lead to interference effects. As a consequence, afterbody flight data are a very valuable source of validation data. So far, only a few afterbody aeroheating flight data are available and the majority comes from 60s flights, like from the Mercury and Apollo programs. They can still be used for validation purposes, especially since uncertainties in afterbody aeroheating predictions obtained with numerical tools stay quite large.

\subsection{The Mercury Project}

The Mercury project ran from 1958 up to 1963 and its objective was to send a man into Earth orbit before returning him safely. The search for an appropriate design capable of ensuring the integrity of the structure and the survivability of the crew was a major priority which led to several flight tests and experiments.

At the beginning of the program, a Mercury prototype capsule protected by a fiberglassphenolic ablative heatshield was launched in 1959. For this flight which was nicknamed BigJoe, the forebody and the afterbody were equipped with many sensors and thermocouples (TC). However, because of significant angle of attack oscillations, the data are not usable for validation studies [3]. Following the first experiment, four Mercury-Atlas (MA) vehicles (Fig. 9) were launched in the entry conditions summarized in Table 2. In February 1961, the MA-2 made a reentry with an apogee of $185 \mathrm{~km}$ and a range of $1257 \mathrm{~km}$. In November 1961, the Ma-5 flight completed two orbits followed by the MA-7 manned 3 orbit mission launched in May 1962. Finally in October 1961, the MA-8 manned 6 orbit mission was launched with virtually identical altitude and velocity histories as those of MA-5 [33].

\begin{tabular}{cccccc}
\hline \hline Flight Id. & Entry date & Afterbody TC number & $U_{\infty}(\mathrm{km} / \mathrm{s})$ & $\alpha$ (deg.) & $\gamma$ (deg.) \\
\hline MA-2 & 21 Feb. 1961 & 17 & 5.5 & 12.5 & - \\
MA-5 & 29 Nov. 1961 & 9 & 7.4 & 0 & -1 \\
MA-7 & 24 May 1962 & 12 & 7.4 & 0 & -1 \\
MA-8 & 3 Oct. 1962 & 16 & 7.4 & 0 & -1 \\
\hline \hline
\end{tabular}

Table 2: Entry flight conditions and instrumentation of the four Mercury-Atlas (MA) vehicles (adapted from $[3],[33])$. 


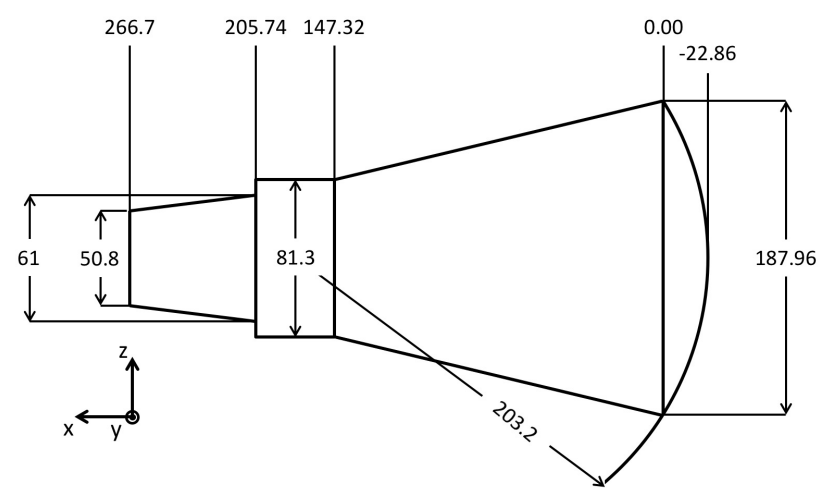

Figure 9: Schematic of the Mercury capsule configurations (adapted from [3]). Dimensions in $\mathrm{cm}$.

As it can be seen in Table 2, the four flights were suborbital flights with maximum velocities ranging from 5.5 to $7.4 \mathrm{~km} / \mathrm{s}$. The detailed trajectory data of each flight are presented in [33]. The four capsules were equipped with chromel-alumel TCs located at the inner surface of the outer skin of the vehicle. Each vehicle was instrumented with respectively 17, 9, 12 and 16 afterbody TCs. The data measured by the TCs consist of raw temperatures histories that were processed by a data reduction program in order to reconstruct the corresponding surface heat fluxes histories. The data reduction routines as well as the precise TCs locations are fully described by Murphy [33]. An error analysis on the principal sources of errors is also presented in this report and states that if all the sources were to act in the same direction, the maximum error would be smaller than $50 \%$ for the period of reliable data reduction. An estimation of such period is only given for the MA-5 flight and corresponds to the 150-420 s time interval.

Murphy's report gives temperatures and heat fluxes histories in both graphical and tabulated forms. For each flight, the heat fluxes were extracted for time periods that encompass the 120-80 km altitude range which corresponds to the rarefied regime. Examples of such results enabling the analysis of the reentry configuration are given in Figures 10 and 11 where $t$ designates the time from the nominal altitude of $122 \mathrm{~km}$ (i.e $400 \times 10^{3} \mathrm{ft}$ ).

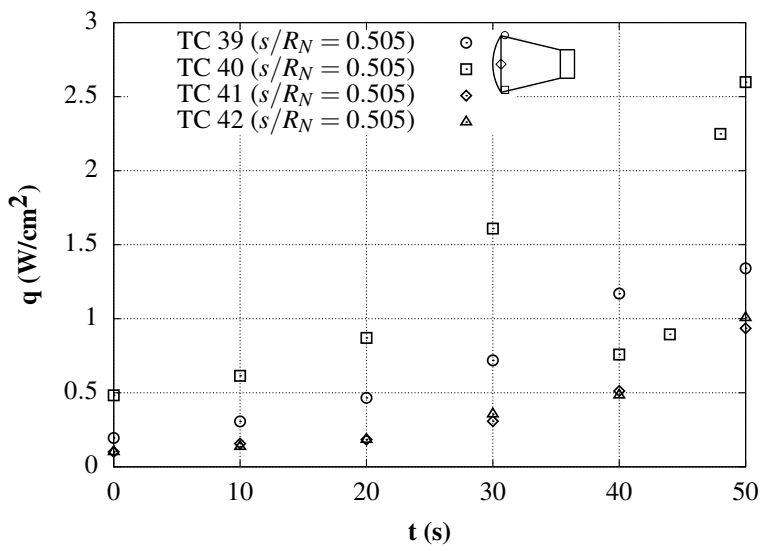

(a) Rarefied heating data for the MA-2 capsule recorded at four TC locations. TCs 41 and 42 are placed at the same location.

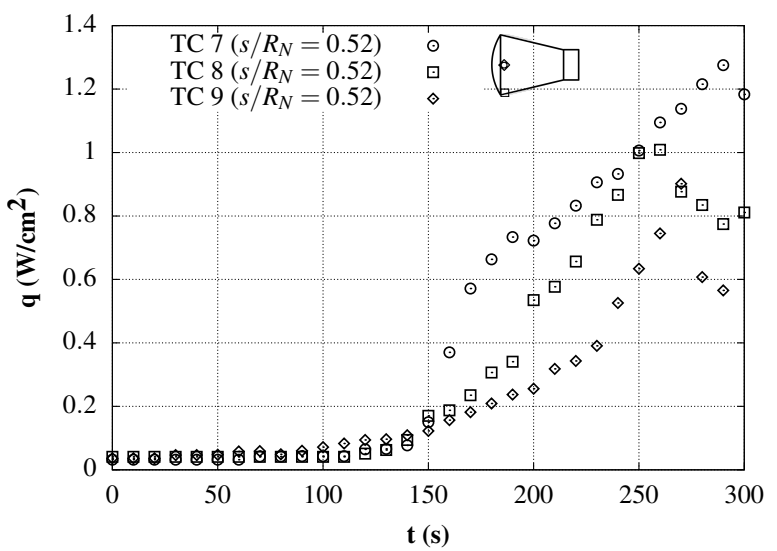

(b) Rarefied heating data for the MA-7 capsule recorded at three TC locations. TCs 7 and 9 are symmetrical to one another referring to the y axis.

Figure 10: Rarefied heating data for the MA-2 and MA-7 test flights (extracted from [33]).

No inertial measurement units (IMU) were used for these flights, which is why no precise angle of attack histories are available. However, Murphy indicates that the MA-2 probe 


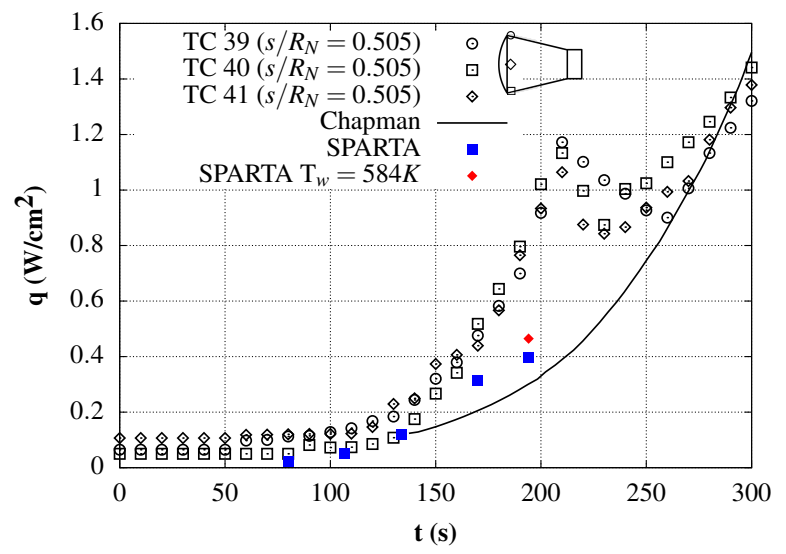

(a) Rarefied heating data for the MA-5 capsule recorded at three TC locations.

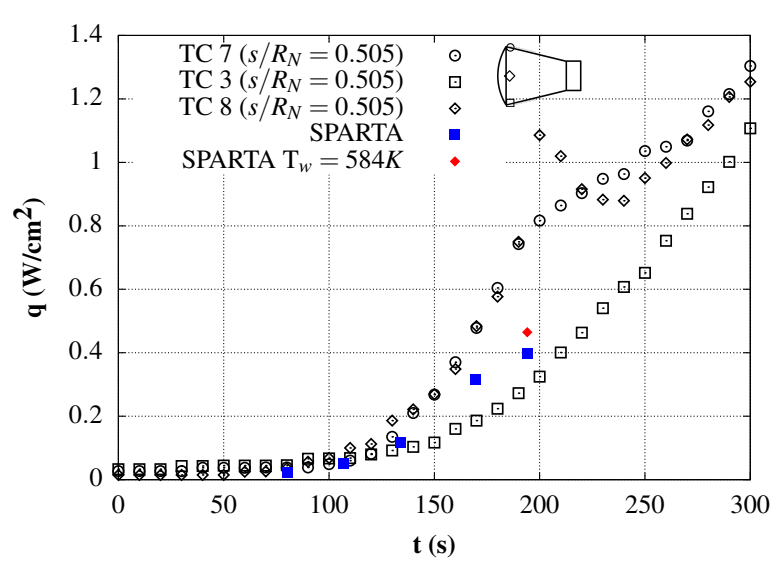

(b) Rarefied heating data for the MA-8 capsule recorded at three TC locations.

Figure 11: Rarefied DSMC and flight heating data for the MA-5 and MA-8 test flights (extracted from [33]).

entered with a large angle of attack (AoA) $\alpha=12.5^{\circ}$ and made a ballistic entry at this incidence for the major portion of it. This tendency is visible on the heat flux values of Figure 10a since the heat flux recorded by the TC number 40 is the largest. According to Murphy, except for the flight MA-2, the three other vehicles were essentially flying at zero AoA over the whole trajectory. For the MA-5 flight, the TCs number 39, 40 and 41 are located in the same $(x, z)$ plane. For the flight MA-7, Figure $10 \mathrm{~b}$ as well as the complementary data presented in [33] show significant discrepancies between TCs placed at the same distance from the probe's nose. These results suggest that the vehicle probably flew in non-nominal conditions. The flights MA-5 and MA-8 occurred with quasi-identical trajectories. Besides the TC 3 of flight MA-8 whose values seem inconsistent (Figure 11b), the analysis of the flight data of both flights show little dispersions for TCs at similar distances from the probe's nose. Hence, it was deduced that those two flights effectively entered Earth atmosphere with small AoA and AoS (angle of sideslip).

In order to investigate those data, we performed a set of five SPARTA DSMC simulations with the freestream and numerical conditions respectively given in Tables 3 and 4 . The same models as those described in section 3.2 were employed and the results are superimposed to the flight data in Figures 11 and 12. The Chapman curve in Figure 11a corresponds to Chapman's theory which gives the afterbody heat flux for a laminar, detached flow [34] and was extracted from [33].

\begin{tabular}{ccccccccc}
\hline \hline $\mathrm{t}(\mathrm{s})$ & Altitude & $U_{\infty}(\mathrm{km} / \mathrm{s})$ & $T_{\infty}(\mathrm{K})$ & $n_{\infty}\left(\cdot / \mathrm{m}^{3}\right)$ & $\mathrm{X}_{\mathrm{N}_{2}}$ & $\mathrm{X}_{\mathrm{O}_{2}}$ & $\mathrm{X}_{\mathrm{O}}$ & $K n$ \\
\hline 80 & 110 & 7.47 & 244.7 & $2.1305 \times 10^{18}$ & 0.7700 & 0.1232 & 0.1068 & 0.39 \\
107 & 106 & 7.47 & 214.8 & $4.1894 \times 10^{18}$ & 0.7796 & 0.1475 & 0.0729 & 0.20 \\
134 & 102 & 7.47 & 198.5 & $8.3982 \times 10^{18}$ & 0.7849 & 0.1678 & 0.0473 & 0.10 \\
170 & 96 & 7.47 & 188.5 & $2.4283 \times 10^{19}$ & 0.7865 & 0.19351 & 0.0200 & 0.035 \\
194 & 92 & 7.47 & 188.1 & $4.9646 \times 10^{19}$ & 0.7873 & 0.2056 & 0.0071 & 0.017 \\
\hline \hline
\end{tabular}

Table 3: Atmospheric conditions for the MA-5 and MA-8 flights (extracted from [35]).

The results show that the DSMC predictions underestimate the reconstructed heat fluxes on the afterbody conical portion of the probe but they follow the same trend as the flight data and remain in the $50 \%$ range of estimated error for $t>150 \mathrm{~s}$. On the cylindrical portion of the probe, a good agreement is observed between the numerical results and the reconstructed values. The DSMC results are quite encouraging and the discrepancies with the reconstructed values can be due to the following explanations. 


\begin{tabular}{cccccc}
\hline \hline Altitude $(\mathrm{km})$ & $\lambda_{\infty}^{\mathrm{HS}} / \Delta_{x, y}$ & Adaptive refinement & Number of particles & $\tau_{\text {coll }} / \Delta t$ & $T_{w}(\mathrm{~K})$ \\
\hline 110 & 10 & Yes & $90 \times 10^{6}$ & 7.3 & 807 \\
106 & 6 & Yes & $90 \times 10^{6}$ & 6 & 961 \\
102 & 4 & Yes & $100 \times 10^{6}$ & 5.7 & 1148 \\
96 & 3 & Yes & $180 \times 10^{6}$ & 3.7 & 1384 \\
92 & 2.5 & Yes & $470 \times 10^{6}$ & 2.7 & 1515 \\
92 & 2.5 & Yes & $470 \times 10^{6}$ & 1.7 & 584 \\
\hline \hline
\end{tabular}

Table 4: Numerical parameters for the SPARTA simulations of 5 flight points of the MA-5 and MA-8 flight tests trajectory.

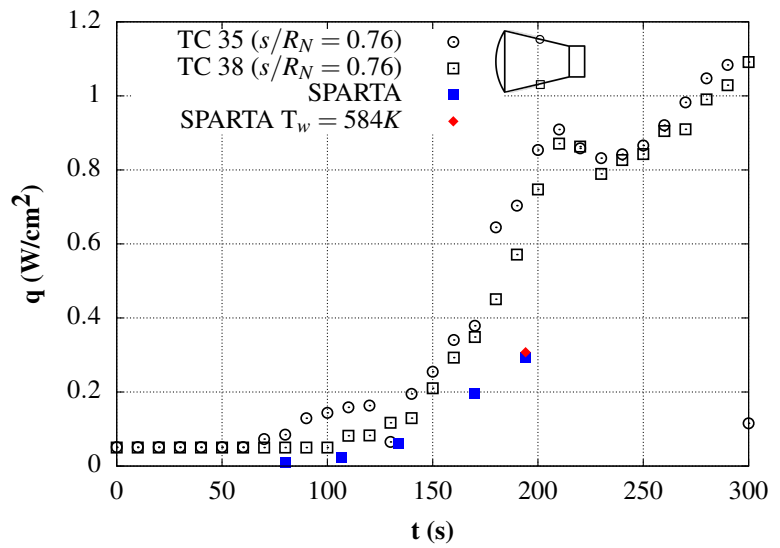

(a) Rarefied heating data for the MA-5 capsule at two locations of the conical section.

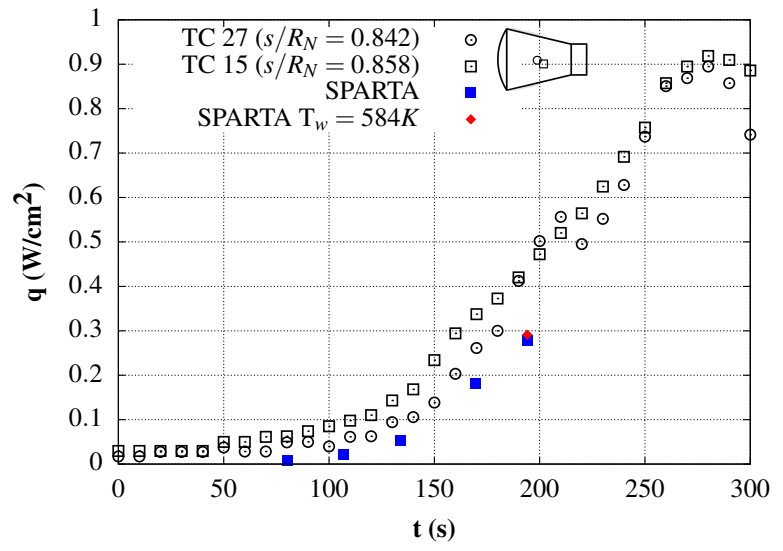

(b) Rarefied heating data for the MA-8 capsule at two locations of the conical section.

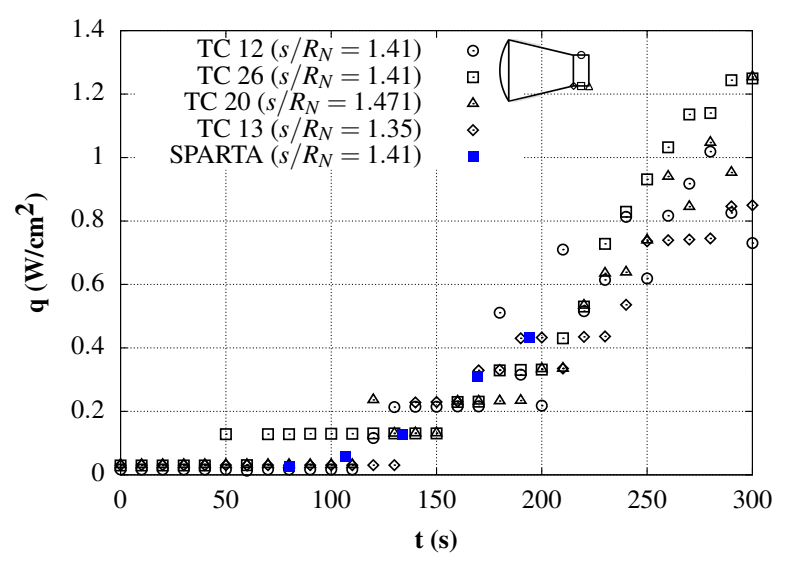

(c) Rarefied heating data for the MA-8 capsule at four locations of the cylindrical section.

Figure 12: Rarefied DSMC versus flight data for the MA-5 and MA-8 test flights (extracted from [33]).

First, because the heat load undergone by the different regions of the probe are highly inhomogeneous, the use of an homogeneous wall temperature computed from the convective heat flux at the stagnation point of the probe might be inappropriate. In order to evaluate the influence of the wall temperature, two simulations with different wall temperatures were conducted for the lowest altitude point (at an altitude of $92 \mathrm{~km}$ and $t=194 \mathrm{~s}$ ), which is in the rarefied slip regime. For the first simulation performed, the wall temperature was computed from the radiative equilibrium with the convective heat flux obtained from the Sutton correlation leading to $T_{w}=1515 \mathrm{~K}$. For the second simulation, the radiative equilibrium was computed with the convective heat flux taken as an average value of the afterbody reconstructed heat fluxes leading to $T_{w}=584 \mathrm{~K}$. As shown in Figure 11a, only the heat flux computed near the shoulder of the probe is sensitive to the choice of the wall 
temperature. The influence of the wall temperature seems to decrease when the distance with the stagnation point increases, as illustrated by Figures 11a and 12a, at $t=194 \mathrm{~s}$. Indeed, the relative discrepancy between the wall heat flux obtained with the two temperatures is around $17 \%$ near the shoulder and $5 \%$ in the middle of the conical section. The ideal boundary condition would be to use :

1. For the forebody : a wall temperature computed from a radiative equilibrium

2. For the afterbody : a temperature distribution interpolated from the temperature measurements reported by Murphy

However, even if the use of a wall temperature distribution would locally give results closer to the experimental measurements, the results presented herein suggest that the difference between the DSMC and the experimental results at $t=194 \mathrm{~s}$ would remain significant and that the effect of the wall temperature cannot be considered as the major source for this discrepancy.

Another possible source of divergence comes from the fact that the two flights did not occur on the same date. This indicates that atmospheric fluctuations may have significantly influenced the TCs measurements and could explain the slight differences in amplitude between the two flight data. Since the simulations are based on the Jacchia model which is an averaged atmospheric model, such fluctuations cannot be captured by our computations.

In the same way, the fact that the vehicles were not carrying on board IMU implies that only the theoretical trajectory is known. This means that significant deviations from the nominal trajectory could have occurred without being noticed which also raises the uncertainty on the freestream conditions we used.

Finally, an additional limitation stems from the data reduction procedure which assumed that the afterbody outer skin was thin enough to be neglected. This assumption enables to compute the heat fluxes from the TCs temperature in a direct fashion whereas an inverse method taking into account the TPS thickness would have been more precise. Contrary to Mercury, the Apollo AS-202 module was carrying an IMU and was equipped with calorimeters, the flight data are then free from such concerns.

\subsection{The Apollo Program}

Following President Kennedy's speech expressing America's objective of landing a man on the Moon before the end of the 60's decade, two new space projects were launched. The Gemini and the Apollo programs started respectively in 1963 and 1961. The accumulated techniques and knowledge during the previous projects and the Flight Investigation of ReEntry (FIRE) research program finally led to the Apollo Command Module (CM) illustrated in Figure 13.

In order to evaluate the module heat-shield, a series of test flights were conducted between 1966 and 1968 (Table 5). One of these flights objectives was to evaluate the capacity of analytical models combining wind-tunnel experiments and theoretical models to predict thermal solicitations acting on the vehicle [36]. Another objective was to evaluate the thermal protection system (STS) performance [37]. The test flight trajectories are given in [36].

With the improvements of measurement techniques a large quantity of data were collected during these flights (pressure, convective and radiative heat fluxes ...). Moreover, among the four test flights, the AS-201 module was the only one which was not equipped with an IMU. However, Apollo flight data were only used and published for CFD-code validation purposes, 50 years later, by Wright et al. [38] and Walpot et al. [39]. In DSMC, the simulation of the aerodynamic coefficients of the Apollo 6 command module was first completed by Moss [40], [41]. It then became a numerical validation case [42], [43], [44] but no aerodynamic 


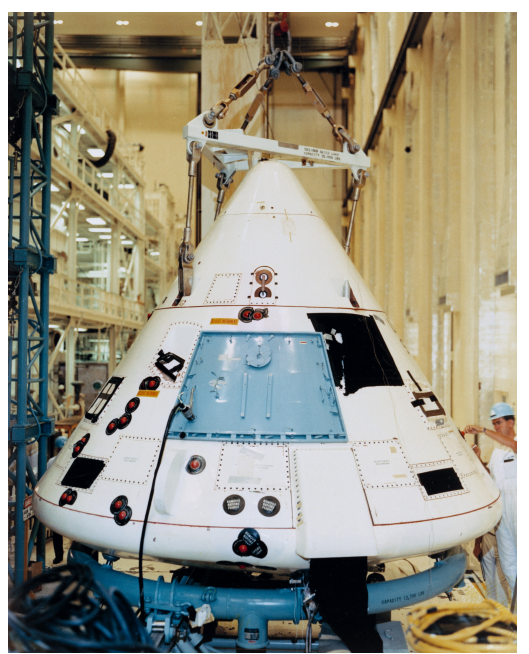

(a) AS-202 command module before flight.

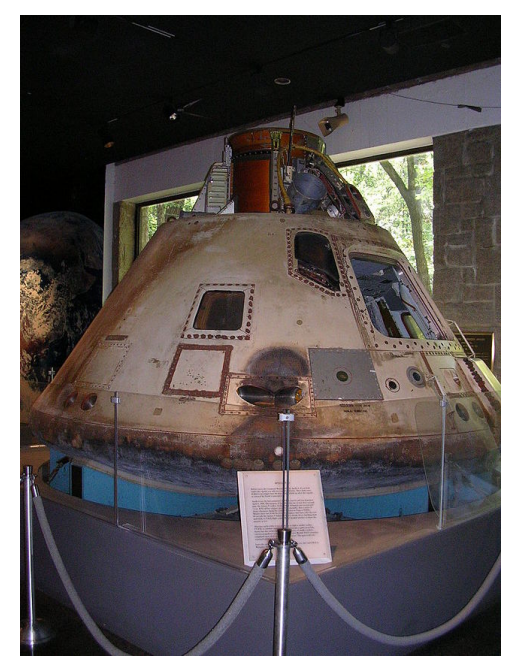

(b) Apollo 6 command module after flight (Fernbank Science Center Atlanta).

Figure 13: Pictures of two Apollo test modules before and after atmospheric reentry (credits: NASA).

\begin{tabular}{cccccc}
\hline \hline Flight Id. (date) & Velocity $(\mathrm{km} / \mathrm{s})$ & $\alpha$ (deg.) & $\gamma($ deg. $)$ & Max. decel. $(\mathrm{g})$ & $q_{\max }^{\text {th }}\left(\mathrm{W} / \mathrm{m}^{2}\right)$ \\
\hline AS-201 $(02 / 26 / 1966)$ & 7.67 & 20 & -8.6 & 14.3 & 186 \\
AS-202 $(08 / 25 / 1966)$ & 8.29 & 18 & -3.5 & 2.4 & 91 \\
Apollo 4 $(11 / 09 / 1967)$ & 10.73 & 25 & -5.9 & 4.6 & 237 \\
Apollo 6 (04/04/1968) & 9.6 & 25 & -6.9 & 7.3 & 488 \\
\hline \hline
\end{tabular}

$\alpha, \gamma$ and $q_{\max }^{\text {th }}$ respectively denote the angle of attack, the flight path angle and the maximal theoretical heat flux.

Table 5: Reentry flight parameters of the Apollo flight tests (adapted from [38]).

flight data are available for this case of study which stays purely numerical. In fact, for most of these flights, the lack of trajectory data, the sensors malfunction or the high velocities prevent the data exploitation for the rarefied portion of the reentry.

In the present paper, the decision was made to numerically compare SPARTA with other codes for the Apollo 6 test case. Then, the Apollo AS-202 flight experiment was simulated and the results were compared with available flight data.

\subsubsection{Apollo 6: Aerodynamic coefficients simulation}

In 2005, NASA presented its new vision of habited space exploration vehicle based on a new generation of Crew Exploration Vehicle (CEV). Different versions were considered in order to be usable for different tasks such as supplying the International Space Station (ISS) as well as being used for Moon and Mars exploration. The new generation of CEV is based on the Apollo design and efforts were directed towards the construction of ATD database [40]. In this context, Moss worked on the simulation of aerodynamic coefficients of the Apollo command module in the flight conditions of Apollo 6 [40], [41].

Our DSMC simulations were made with SPARTA in the same conditions as those described in Moss' paper [40]. The objective is to simulate the reentry of the Apollo 6 command module (Fig. 14a) between 200 and $65 \mathrm{~km}$. In Moss' work, the rarefied portion of the trajectory (between 200 and $85 \mathrm{~km}$ ) was simulated with the DSMC code DS3V [45]. The DSMC parameters were taken as follows. Energy exchanges are permitted between the translational mode and the rotational and vibrational modes according to the Larsen-Borgnakke model [13]. The rotational number was taken constant $Z_{\text {rot }}=5$ and the vibrational number was modeled with the Millikan-White model [46]. Non-catalytic walls were supposed with 
constant temperature $\left(T_{w}\right)$ computed by Moss [40]. Maxwell's gas-surface interaction with complete thermal accommodation and a 5 species air model were used. Chemical reactions were considered with the Total Collision Energy (TCE) model [1] and Park's reaction rate coefficients [47]. For the continuum portion of the trajectory, CFD simulations were made by Moss with LAURA [48], [49].

The atmospheric conditions are given in Table 6 . For altitudes $\geq 90 \mathrm{~km}$, the Jacchia model [35] was used and the 1962 US Standard model [50] was used for altitudes $<90 \mathrm{~km}$. In accordance with Moss, the Knudsen number was computed with a characteristic length equal to the vehicle's base diameter $\left(D_{b}\right)$ and a mean free path given by the Hard Sphere (HS) collision model:

$$
\lambda_{\infty}^{\mathrm{HS}}=\frac{1}{\sqrt{2} \pi d_{\mathrm{ref}}^{2} n_{\infty}},
$$

with $d_{\text {ref }}=3.78 \times 10^{-10} \mathrm{~m}$. The symmetry plan $(x, y)$ was used to divide by two the computational domain size and a 21970 surface mesh (Figure 14b) was used for the SPARTA simulations which were divided in two sets. In the first set, the rarefaction effect between 200 and $85 \mathrm{~km}$ (bold altitudes in Table 6) were quantified by maintaining a constant velocity $U_{\infty}=9.6 \mathrm{~km} / \mathrm{s}$ and angle of attack $\alpha=-25^{\circ}$. In the second set, angle of attack effects were evaluated by varying $\alpha$ between 0 and $180^{\circ}$ at an altitude of $105 \mathrm{~km}$. Moss extended the angle of attack study from 180 down to $-180^{\circ}$ and he made a third set of study with varying velocity at an altitude of $105 \mathrm{~km}$ and an angle of attack of $-25^{\circ}$. These results show the linear influence of velocity on the aerodynamic coefficients [40].

For the SPARTA computations the time step is chosen smaller than the minimum value of the inter-collision time $\tau_{\text {coll }}$ inside the computational domain. The number of particles is chosen as described by Moss for the altitudes $\geq 105 \mathrm{~km}$ but because the values for the lower altitudes were judged too small to reach statistical convergence, a greater number of particles was taken for the 95 and $85 \mathrm{~km}$ altitudes (Table 7). The initial computational mesh was built so that the mean cell size $\Delta_{\mathrm{x}, \mathrm{y}, \mathrm{z}}$ is smaller than the freestream mean free path $\lambda_{\infty}^{\text {HS }}$. Adaptive mesh refinement was used and the third column of Table 7 indicates if it was triggered. Because of computational resource constraints, the refinement for the two lowest altitudes was limited to a 2 level refinement (initial cells can only be divided once by two in each direction only) and the refinement was limited to a rectangular region before the capsule front face.

\begin{tabular}{ccccccccc}
\hline \hline Alt. $(\mathrm{km})$ & $n_{\infty}\left(\cdot / \mathrm{m}^{3}\right)$ & $\rho_{\infty}\left(\mathrm{kg} / \mathrm{m}^{3}\right)$ & $\mathrm{X}_{\mathrm{O}_{2}}$ & $\mathrm{X}_{\mathrm{N}_{2}}$ & $\mathrm{X}_{\mathrm{O}}$ & $T_{\infty}(\mathrm{K})$ & $T_{w}(\mathrm{~K})$ & $K n$ \\
\hline $\mathbf{2 0 0}$ & $9.00 \times 10^{15}$ & $3.28 \times 10^{-10}$ & 0.0315 & 0.4548 & 0.5138 & 1026 & 234 & 44.74 \\
$\mathbf{1 7 0}$ & $2.27 \times 10^{16}$ & $8.78 \times 10^{-10}$ & 0.0435 & 0.5482 & 0.4083 & 892 & 300 & 17.74 \\
$\mathbf{1 5 0}$ & $5.31 \times 10^{16}$ & $2.14 \times 10^{-9}$ & 0.0546 & 0.6156 & 0.3298 & 733 & 373 & 7.59 \\
$\mathbf{1 3 0}$ & $1.94 \times 10^{17}$ & $8.21 \times 10^{-8}$ & 0.0709 & 0.6911 & 0.238 & 500 & 524 & 2.07 \\
$\mathbf{1 1 5}$ & $9.86 \times 10^{17}$ & $4.36 \times 10^{-8}$ & 0.0978 & 0.7539 & 0.1484 & 304 & 795 & 0.408 \\
110 & $2.12 \times 10^{18}$ & $9.61 \times 10^{-8}$ & 0.1232 & 0.7704 & 0.1064 & 247 & 920 & 0.190 \\
$\mathbf{1 0 5}$ & $5.09 \times 10^{18}$ & $2.36 \times 10^{-7}$ & 0.1581 & 0.7832 & 0.0587 & 208 & 1029 & 0.081 \\
$\mathbf{1 0 0}$ & $1.19 \times 10^{19}$ & $5.58 \times 10^{-7}$ & 0.1768 & 0.7844 & 0.0388 & 194 & 1146 & 0.0338 \\
$\mathbf{9 5}$ & $3.12 \times 10^{19}$ & $1.48 \times 10^{-6}$ & 0.2004 & 0.7869 & 0.0127 & 189 & 1295 & 0.0139 \\
90 & $7.08 \times 10^{19}$ & $3.38 \times 10^{-6}$ & 0.2091 & 0.7875 & 0.0035 & 188 & 1436 & 0.0057 \\
$\mathbf{8 5}$ & $1.65 \times 10^{20}$ & $7.96 \times 10^{-6}$ & 0.2372 & 0.763 & 0.0 & 181 & 1598 & 0.0024 \\
\hline \hline
\end{tabular}

Table 6: Atmospheric conditions for the Apollo 6 module (extracted from [40]).

The quantities of interest in this study are the aerodynamic coefficients of axial force $\left(C_{A}\right)$, of normal force $\left(C_{N}\right)$, the pitching moment at the center of gravity $\left(C_{m, c g}\right)$ and the 


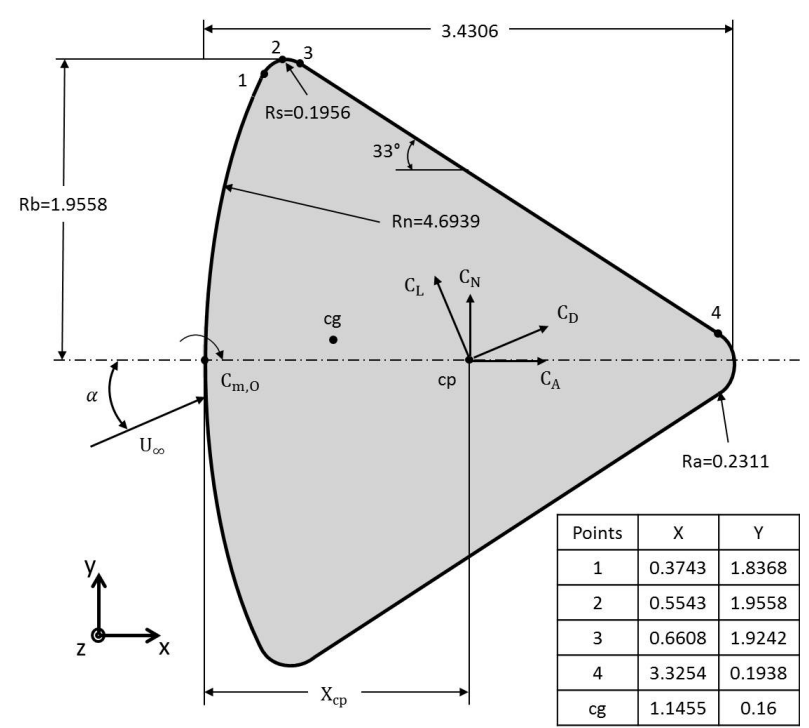

(a) Schematic of the Apollo command module and aerodynamic coordinate system (adapted from [40]). Dimensions in $\mathrm{m}$.

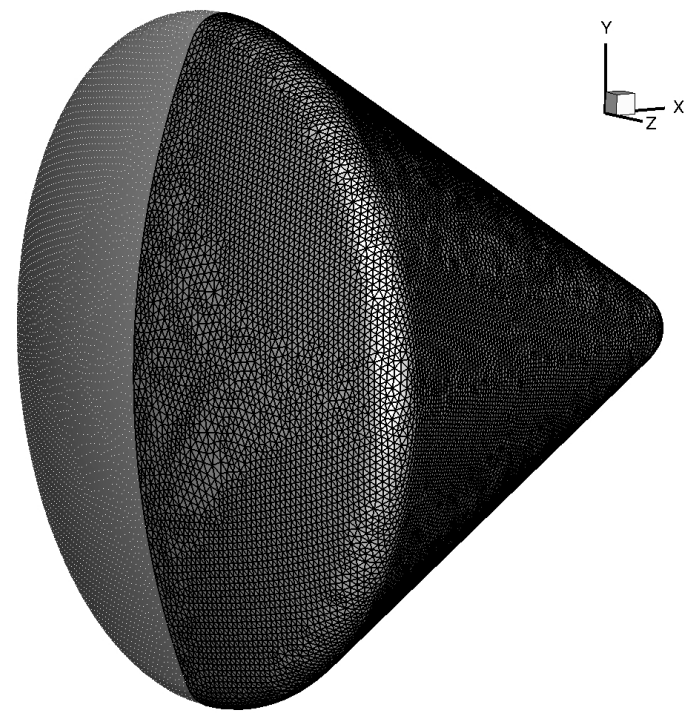

(b) Frontal view of Apollo surface mesh used in the SPARTA computations.

Figure 14: View of the simplified geometry and the surface mesh of the Apollo command module.

\begin{tabular}{ccccc}
\hline \hline Altitude $(\mathrm{km})$ & $\lambda_{\infty} \mathrm{HS} / \Delta_{\mathrm{x}, \mathrm{y}, \mathrm{z}}$ & Adaptive refinement & Number of particles & $\tau_{\text {coll }} / \Delta t$ \\
\hline 200 & 87.5 & No & $2 \times 10^{6}$ & 9 \\
170 & 41.6 & No & $2.6 \times 10^{6}$ & 16 \\
150 & 29.7 & No & $3.1 \times 10^{6}$ & 5.6 \\
130 & 16.2 & Yes & $4.3 \times 10^{6}$ & 5.1 \\
115 & 7.9 & Yes & $6.6 \times 10^{6}$ & 4.2 \\
105 & 3.2 & Yes & $21.5 \times 10^{6}$ & 3.9 \\
95 & 1.65 & Yes & $200 \times 10^{6}$ & 2.0 \\
85 & 0.4 & Yes & $1000 \times 10^{6}$ & 1.7 \\
\hline \hline
\end{tabular}

Table 7: Numerical parameters for the SPARTA simulations of the Apollo 6 module.

position of the center of pressure $\left(X_{c p}\right)$ with the coordinate system and nomenclature described in Figure 14a. For the first set of simulation, Figure 15a shows the evolution of the aerodynamic forces coefficients as a function of the altitude. Figure $15 \mathrm{~b}$ shows the evolution of the moment coefficient and the center of pressure position as a function of the altitude. An excellent agreement is observable between the DS3V and the SPARTA results with differences smaller than $5 \%$. In near-continuum regime, the results discrepancies obtained with the two DSMC codes are due to the difference in particle number and in mesh refinement. Indeed, DS3V is a sequential code which means that computing limitations are greater than for SPARTA. The comparison between the results of SPARTA and LAURA shows significant differences for the center of pressure position and the moment coefficient. This can be explained by the fact that even with SPARTA, spatial convergence is not reached at low altitudes. Indeed, in DSMC the computational memory load depends on the mesh size and spatial convergence can become too demanding to be reached in near continuum conditions.

Regarding the second set of simulation, Figures 16a and 16b show that both DSMC codes give results in close agreement for varying angles of attack and at an altitude of 105 
$\mathrm{km}$. From an aerodynamic perspective, the objective is usually to minimize the pitching moment coefficient to ensure the vehicle's stability. Figure $16 \mathrm{~b}$ indicates that stability would be reached for an angle of attack of around $83^{\circ}$. Moss' results show that on the negative side of the incidence interval, $C_{m, c g}$ is minimal at $-164^{\circ}$. This would mean that in the flight conditions of the Apollo 6 mission, the vehicle was probably statically unstable for the transitional portion of the reentry.

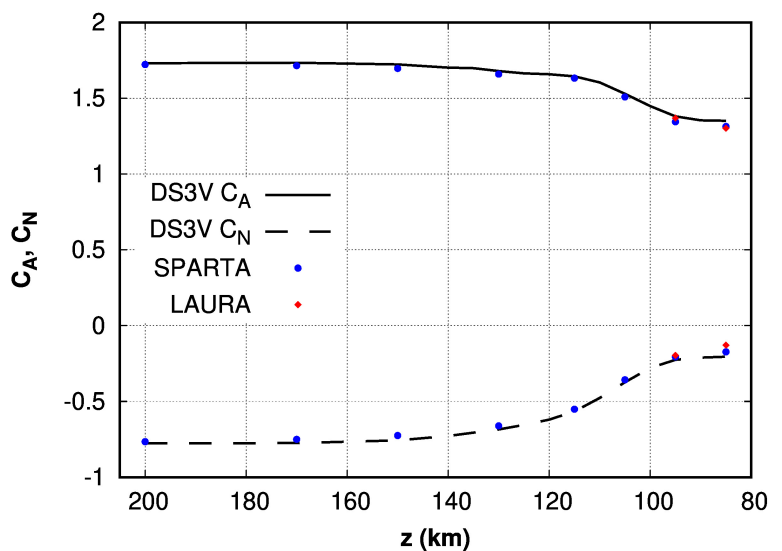

(a) Normal and Axial force coefficients.

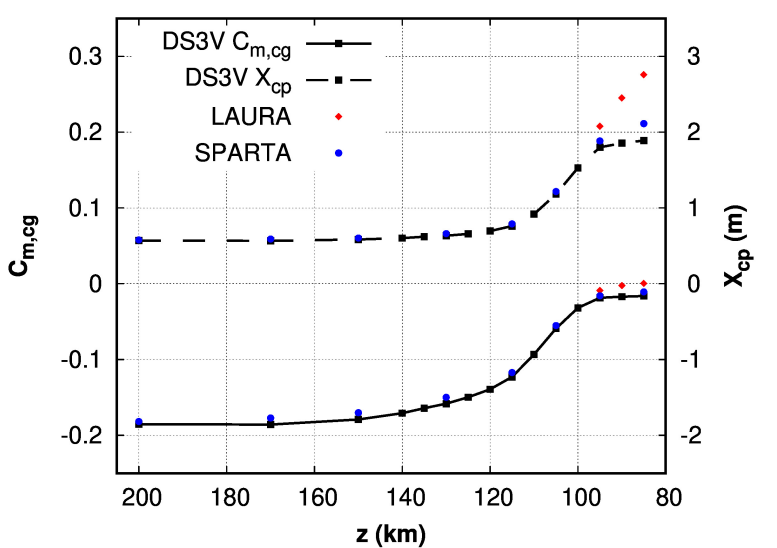

(b) Pitching moment coefficient and center of pressure position.

Figure 15: Evolution of the numerical aerodynamic coefficients of Apollo 6 as a function of the altitude between 200 and $85 \mathrm{~km}$.

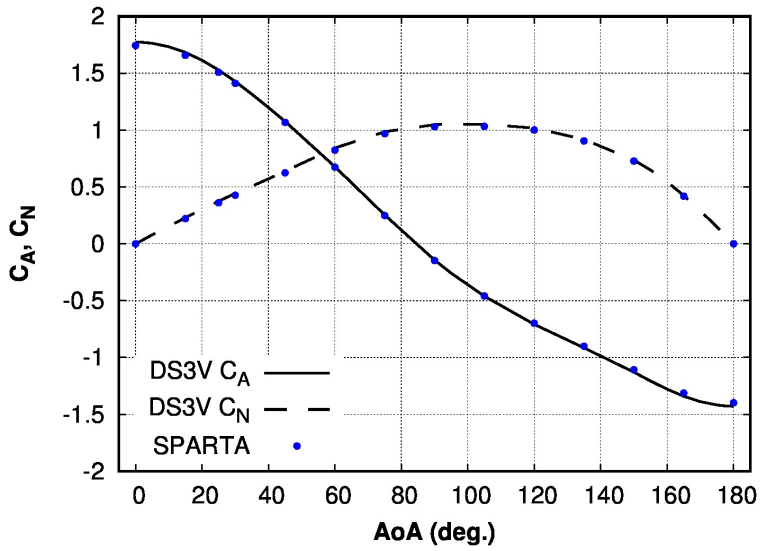

(a) Normal and Axial force coefficients.

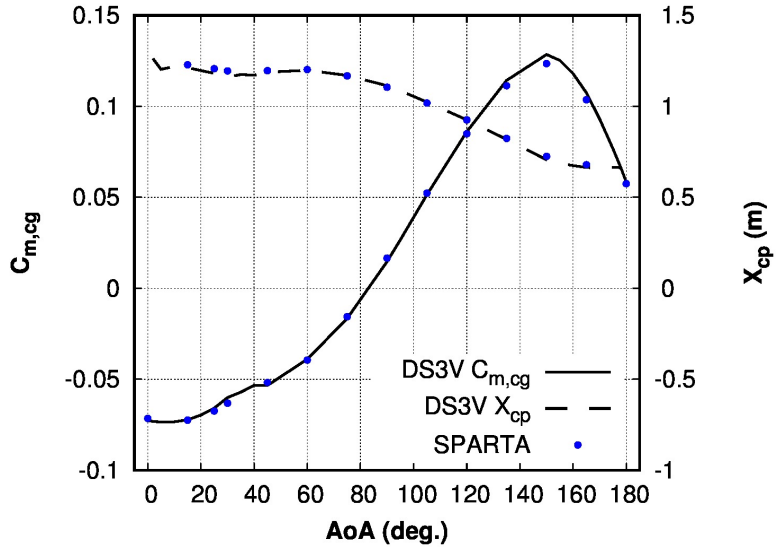

(b) Pitching moment coefficient and center of pressure position.

Figure 16: Evolution of numerical aerodynamic coefficients of Apollo 6 as a function of the angle of attack at an altitude of $105 \mathrm{~km}$.

To conclude on this case of study, the simulation of the aerodynamic coefficients of the Apollo 6 mission as described in Moss' work can be very useful. Indeed, Banyai et al. [51] have based their study of the aerodynamic coefficients of the Intermediate eXperimental Vehicle (IXV) in rarefied regime on Moss' work. They used the simulation of the Apollo 6 command module aerodynamic coefficients as the first step of their study in order to validate their code as well as to better apprehend the simulation of the aerodynamics of the IXV. Finally, even if flight data exist for Apollo 6 and 4 [52], [53], because in lunar return conditions the velocity is particularly high, the probe quickly passes through the rarefied regime. Therefore, not enough data were recorded in this regime to be exploited. 


\subsubsection{Apollo AS-202: Afterbody heat flux simulation}

In this section, the focus is given to the flight Apollo AS-202 which is particularly interesting for several reasons. The AS-202 command module was the first Apollo test vehicle carrying an IMU which means that precise trajectory data (angle of attack, sideslip angle, etc) are available. Moreover, because afterflight observations evidenced little charring during the AS-202 entry [38] and given the freestream conditions (velocity and density) of the transitional portion, all ablative phenomena were assumed negligible in our analysis.

The AS-202 command module is represented in Figure 13a. It was made of a stainless steel structure covered with Avcoat 5026/39G TPS, an ablator described in [3], [38]. The module forebody was instrumented with 12 pressure transducers and 12 calorimeters. Among the pressure transducers, 10 transducers provided usable data but none of the calorimeters. 24 pressure transducers and 23 calorimeters were placed on the afterbody. Only two pressure sensors recorded data just before the parachute opening. Unfortunately, all the pressure measurements correspond to the continuum regime only [54] and the force measured during the low deceleration level phase which encompasses the rarefied portion have uncertainties too high to be usable [55]. Among the afterbody calorimeters, 19 worked correctly (Figure 17). The precise positions of the calorimeters which take into account the thickness of the heatshield are given by Wright et al. [38] and were used in this study.

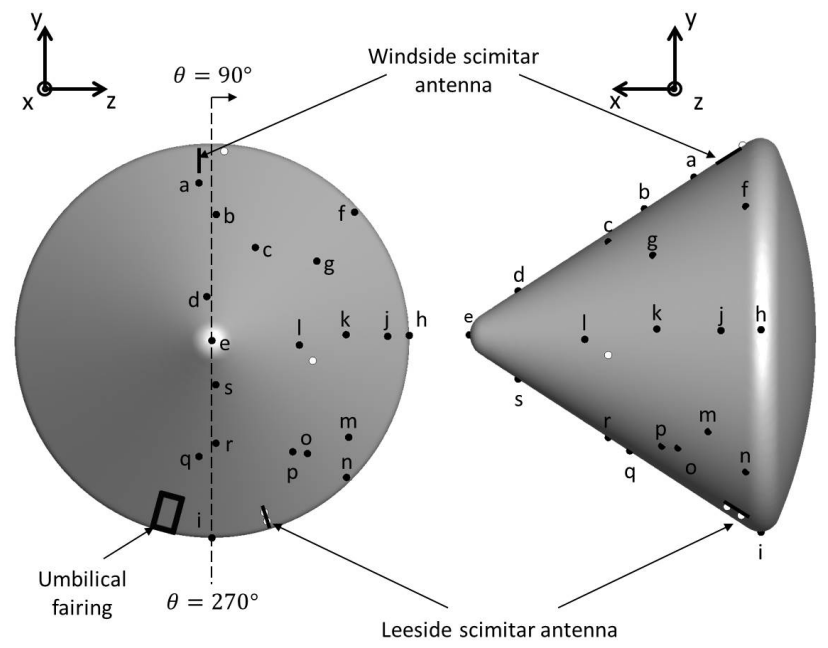

Figure 17: Calorimeters positions on the AS-202 afterbody. White symbols stand for non-working calorimeters (adapted from [38]).

The calorimeters are Gardon gauges described in [56] and designed to measure heat flux smaller than $58 \mathrm{~W} / \mathrm{cm}^{2}$. According to Wright et al., the gauges uncertainty is $\pm 20 \%$ in the flight conditions considered. The NASA technical report does not give other uncertainty estimation so the same assumption was made.

Hillje's report [55] gives the history of the mission aerodynamic quantities which was used to obtain the angle of attack $\alpha$ and the sideslip angle $\beta$. The references [38], [39] were used to extract the velocity and altitude as a function of time. The flight data presented in [54] were extracted from Wright's paper [38] and the study was limited to the time period between the entry point and around $t=4500 \mathrm{~s}$. Hence, a total of 6 flight points evenly spaced by $5 \mathrm{~km}$ were simulated. The flight parameters are summarized in Table 8 .

The values show that the angle of attack ranges from 19 to $25^{\circ}$ and the sideslip angle oscillates between -1.5 and $3.5^{\circ}$ with maximum values for 105 and $85 \mathrm{~km}$. To take into account the sideslip angle, no symmetry plane can be used which means that the computational resources must be multiplied by a factor of two to keep the same precision as for the Apollo 6 simulations. Moreover, for the $85 \mathrm{~km}$ altitude $\left(K n=2.4 \times 10^{-3}\right)$ which corre- 
sponds to near-continuum conditions, multiplying the resources by two is computationally too expensive. Thus, whatever the altitude, no sideslip was taken into account but potential sideslip effects will be discussed in the results analysis.

\begin{tabular}{ccccccc}
\hline \hline Time $(\mathrm{s})$ & Altitude $(\mathrm{km})$ & $K n$ & $U_{\infty}(\mathrm{m} / \mathrm{s})$ & $\alpha\left(^{\circ}\right)$ & $\beta\left(^{\circ}\right)$ & $T_{w}(\mathrm{~K})$ \\
\hline 4373 & 110 & 0.190 & 8261 & 24.85 & 1.2 & 739 \\
4384 & 105 & 0.081 & 8276 & 25.18 & 3.5 & 826 \\
4396 & 100 & 0.0338 & 8291 & 24.58 & 0.4 & 921 \\
4408 & 95 & 0.0139 & 8302 & 19.44 & -1.5 & 1041 \\
4420 & 90 & 0.0057 & 8307 & 19.9 & -0.1 & 1155 \\
4432 & 85 & 0.0024 & 8291 & 18.81 & 2.3 & 1283 \\
\hline \hline
\end{tabular}

Table 8: Selected flight points along the Apollo AS-202 trajectory for the SPARTA simulations.

For this set of simulations, the same atmospheric conditions as those of the Apollo 6 case were used (Table 6). The wall temperature (given in Table 8) was assumed constant on the overall surface of the capsule. It was computed with a bridging function which solves the radiative equilibrium with a convective heat flux between the continuum value given by the Sutton correlation [57], [58] and the free molecular heat flux given in [1]. The constant wall temperature hypothesis is pretty usual in DSMC studies but it might be inappropriate. As for Mercury, the influence of the wall temperature is also discussed in the analysis of the results.

The simulations were made with the same models as those used for the Apollo 6. The same simplified geometry (Figure 14a) was used with the same surface mesh. According to Wright et al. [38], the simplified geometry does not take into account geometrical specificities such as the umbilical fairing and the scimitar antennas illustrated in Figure 17. However, the location of the umbilical fairing (far from the calorimeters) and the small size of the antennas are supposed to be negligible. Furthermore, even though the AS-202 module was not carrying radiometers and that the measurements of the Apollo 4 and Apollo 6 radiometers indicated negligible radiative heat fluxes [38], Johnston and Brandis [59] showed that because of the calorimeters sensitivity and the restricted range of measurable spectrum, significant radiative heat fluxes might still have been applied to the Apollo 4 and 6 afterbodies. However, the conclusions of their paper stands for entries with velocities greater than $10 \mathrm{~km} / \mathrm{s}$. Here, since the velocity is smaller (around $8.3 \mathrm{~km} / \mathrm{s}$ ), it seems reasonable to assume that ionization and radiative effects can be neglected. The relevance of this assumption was finally confirmed by the small $\mathrm{O}$ and $\mathrm{N}$ number densities obtained in the shock layer for the Apollo AS-202 simulations. For the simulation at $85 \mathrm{~km}$, the densities $\left[n_{N}, n_{O}\right]=\left[2 \times 10^{14}, 1 \times 10^{14}\right]$ are far smaller than the typical values $\left[n_{N}, n_{O}\right]=\left[7.50 \times 10^{15}, 2.11 \times 10^{15}\right]$ at $T_{\mathrm{tr}}=5231 \mathrm{~K}$ given by Johnston and Brandis [59].

For their simulations of the AS-202 reentry in the continuum regime, Wright et al. considered a fully catalytic wall. Because of the small amount of monoatomic species in the afterbody region of the capsule in the rarefied regime, the effect of wall catalysis on the wall heat flux was assumed insignificant and no surface reactions were considered.

The numerical parameters used for the SPARTA simulations are summarized in Table 9. They have been chosen in order to respect the usual DSMC stability criteria as much as possible. For the 90 and $85 \mathrm{~km}$ simulations, which correspond to near-continuum conditions, the initial grid is already too heavy to enable any refinement even though the space convergence is not reached. The table also shows numbers of particles much higher than the numbers used for the Apollo 6 case. Indeed, for this case, a minimum number of 500 millions of numerical particles was used. This choice might seem large at first sight but 


\begin{tabular}{ccccc}
\hline \hline Altitude $(\mathrm{km})$ & $\lambda_{\infty}^{\mathrm{HS}} / \Delta_{\mathrm{x}, \mathrm{y}, \mathrm{z}}$ & Adaptive refinement & Number of particles & $\tau_{\text {coll }} / \Delta t$ \\
\hline 110 & 3.7 & Yes & $500 \times 10^{6}$ & 9.3 \\
105 & 3.2 & Yes & $500 \times 10^{6}$ & 4.7 \\
100 & 2 & Yes & $500 \times 10^{6}$ & 3.3 \\
95 & 2 & Yes & $990 \times 10^{6}$ & 2.2 \\
90 & 1 & No & $1000 \times 10^{6}$ & 2.4 \\
85 & 0.6 & No & $1000 \times 10^{6}$ & 1.5 \\
\hline \hline
\end{tabular}

Table 9: Numerical parameters for the SPARTA simulations of the flight points of the Apollo AS-202 trajectory.

contrary to the Apollo 6 case, the objective of these simulations is to compute heat fluxes on the afterbody. Preliminary simulations have shown that the statistic sensitivity of the afterbody heat fluxes is radically more significant than the sensitivity of the aerodynamic coefficients. Such numbers of particles minimize the statistical noise by ensuring a number of particles per cell greater than 15 everywhere in the domain and even in the afterbody area.

The observation of the streamlines showed that the flow remains attached to the probe down to $95 \mathrm{~km}$ and detaches itself between 95 and $90 \mathrm{~km}$. Examples of attached and detached streamlines are illustrated in Figure 18. For the considered altitudes, the Reynolds number of the lowest point is smaller than $3 \times 10^{4}$. As suggested by Wrigth et al. [38], no turbulent effects are expected for such values.

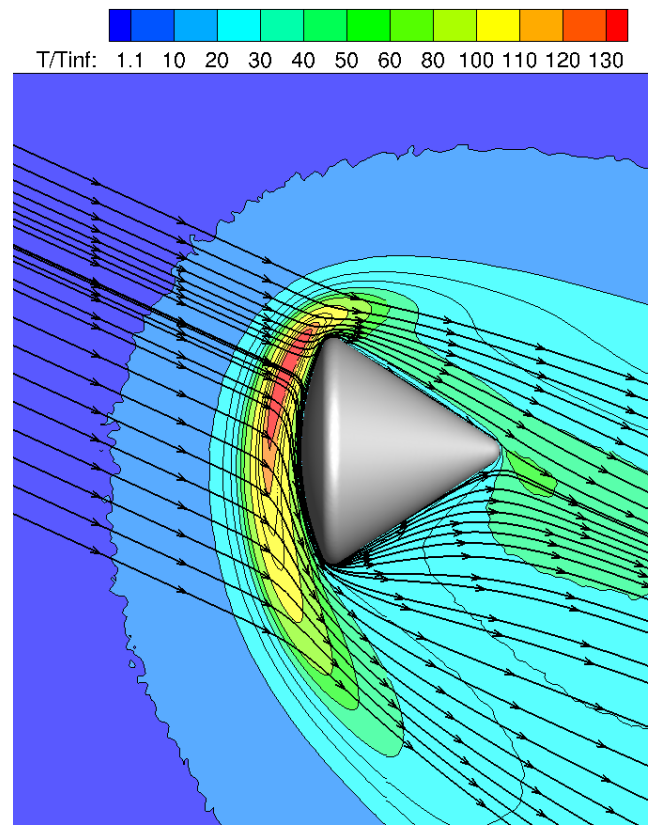

(a) Altitude of $100 \mathrm{~km}, M_{\infty}=29.7, K n=$ 0.0338 and $R e=1.3 \times 10^{3}$.

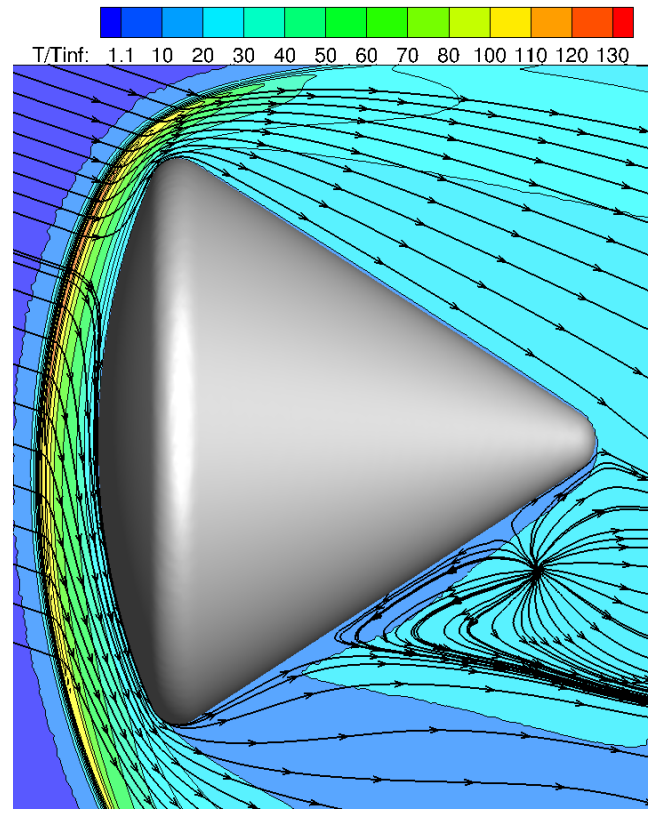

(b) Altitude of $85 \mathrm{~km}, M_{\infty}=30.7, K n=0.0024$ and $R e=1.9 \times 10^{4}$.

Figure 18: DSMC computed nondimensional temperature field and streamlines at 100 and $85 \mathrm{~km}$ during the reentry of the AS-202 module.

The heat fluxes are presented in Figure 19. These results show that for the calorimeters located on the windside, where the heat fluxes are the more severe, a good agreement is observed. More generally, for 14 of the 19 calorimeters, the numerical results are in the $\pm 20 \%$ confidence interval. The five calorimeters which show the biggest differences are the $\mathbf{e}, \mathbf{s}, \mathbf{m}, \mathbf{n}$ and $\mathbf{i}$ calorimeters. The calorimeter $\mathbf{e}$ is particular in that it is located 
at the top of the apex. This means that the discrepancies between the results could be due to the geometrical differences between the actual geometry and the way the apex was simplified. The calorimeter $\mathbf{s}$ is located on the leeward of the capsule and is symmetric to d located on the windward. However, both calorimeters recorded heat fluxes of the same order of magnitude. This observation also stands for the calorimeter $\mathbf{n}$ symmetric to $\mathbf{f}$. In the same way, the heat flux measured by the calorimeter $\mathbf{m}$ is significantly greater then neighboring calorimeters $\mathbf{o}$ and $\mathbf{p}$ but is surprisingly close to the heat flux measured by $\mathbf{j}$ located on the windward. However, due to important angles of attack $(18.81<\alpha<25.18)$ of the considered flight points, the temperature field and the flow topology around the capsule are very different in the leeward and windward regions of the afterbody (Figure 18). Consequently, it is hard to understand why the heat fluxes recorded by some calorimeters are equivalent in the windward and leeward sides. Such anomalies could indicate that for those calorimeters, the uncertainty of measurements is greater than the estimated $20 \%$ or that they malfunctioned during the rarefied regime. For the calorimeter $\mathbf{i}$, the numerical results strongly underestimate the measurements.

This difference could be caused by the critical location of this calorimeter which stands at the shoulder of the module. Numerically, the heat flux is very sensitive to the surface element where the heat flux is considered and can vary between $0.7 \mathrm{~W} / \mathrm{cm}^{2}$ up to $1.3 \mathrm{~W} / \mathrm{cm}^{2}$ for two adjacent surface elements. This puts into perspective the strong disagreement with the measurements.

For most calorimeters, the computed value at the lowest altitude seems incorrect, with a drop more pronounced than expected. This tendency is likely due to the spatial convergence which is not reached for the reasons mentioned previously. In addition, for the 90 and 85 $\mathrm{km}$ altitudes, the flow is detached and a recirculation zone appears behind the probe. The separated wake flow being likely unsteady, the reliability of the heat flux at these altitudes could be consolidated through the extension of the sampling procedure or by performing the average of an ensemble of identical simulations. Given our computational resources, this altitude marks the limit of our DSMC capacities for the computation of the Apollo afterbody heat fluxes.

Since the differences are not specific to the 105 and $85 \mathrm{~km}$ altitudes, where the AoS is maximal, but also observed for altitudes with very small AoS, it was concluded that the failure to take into account the sideslip angle $\beta$ has a small impact on the results.

As mentioned earlier, for the DSMC simulations, the wall temperature was assumed constant and homogeneous on the vehicle surface. However, even in the rarefied regime the wall temperature is inhomogeneous. As seen with the simulations of Mercury, the wall temperature can have a non negligible influence on the wall heat flux for points located near the shoulder region. At an altitude of $85 \mathrm{~km}\left(K n=2.4 \times 10^{-3}\right)$, the use of a modified Sutton convective heat flux (with an enthalpy corrective factor) gives a wall temperature $T_{w}=713 \mathrm{~K}$ which is $44 \%$ smaller than the one used for the DSMC simulations. Thus, a new simulation was made with this temperature and heat fluxes variations were observed: from $2 \%$ for the sensors $\mathbf{a}, \mathbf{d}, \mathbf{j}$ and $\mathbf{r}$ up to $28 \%$ for the sensor $\mathbf{e}$ on the apex. Since small deviations of the order of $50 \mathrm{~W} / \mathrm{m}^{2}$ can represent variations of $10 \%$ for some sensors, for complex 3D simulations, such change could be the result of statistical noise. Therefore, the relation between the calorimeter's location and the sensitivity to the wall temperature can not be reaffirmed with those results. However, the discrepancies confirm that the wall temperature can have a non negligible impact on the computed afterbody heat fluxes and simulations with a variable wall temperature might give more realistic results. In SPARTA, implementing such functionality requires to handle the synchronization of the changing temperature across the processors which may share ownership of surface elements. This 
has not been implemented in the 2019 version of SPARTA but is under consideration by Sandia Laboratory and might be available in its upcoming versions.

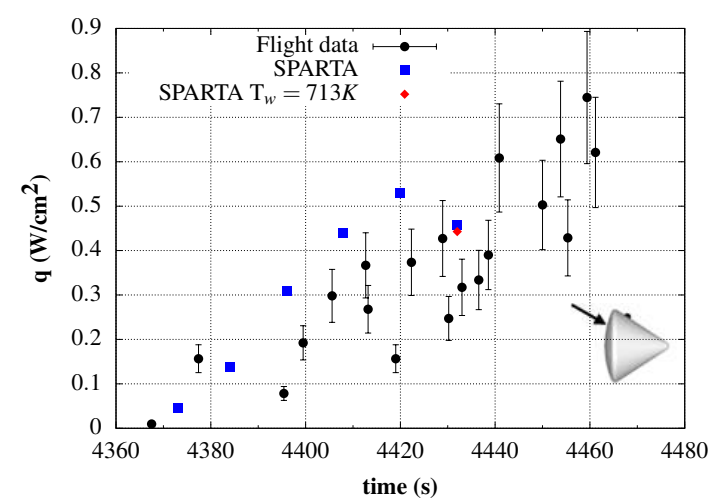

(a) Calorimeter a

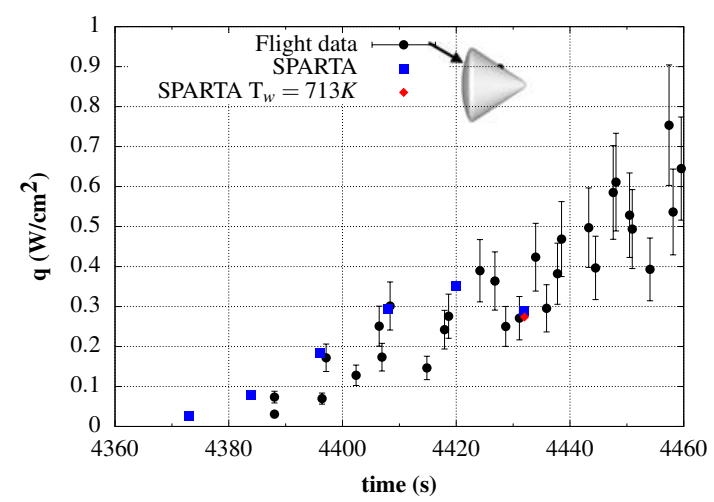

(c) Calorimeter c

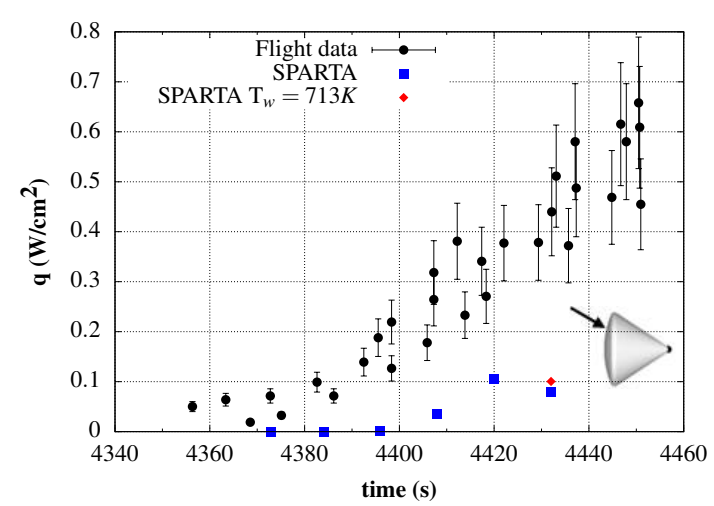

(e) Calorimeter e

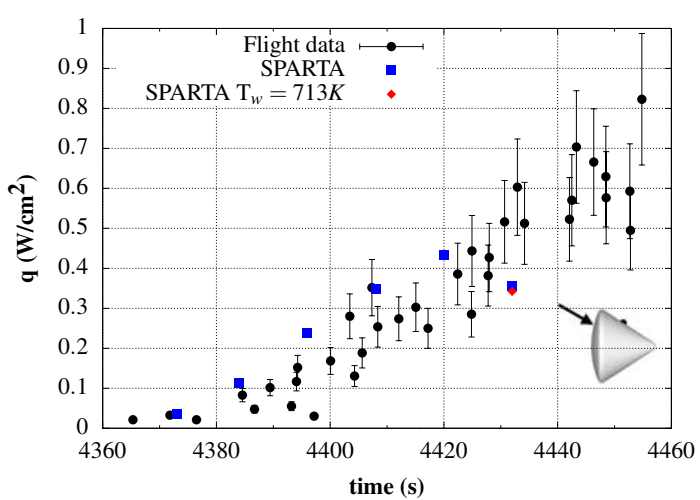

(b) Calorimeter b

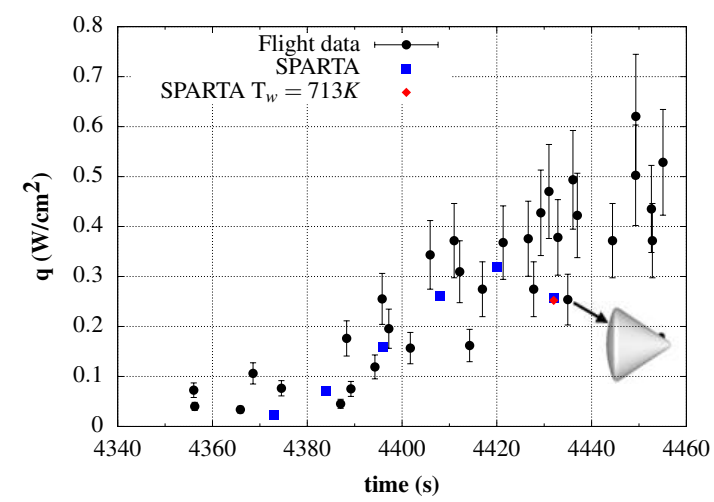

(d) Calorimeter d

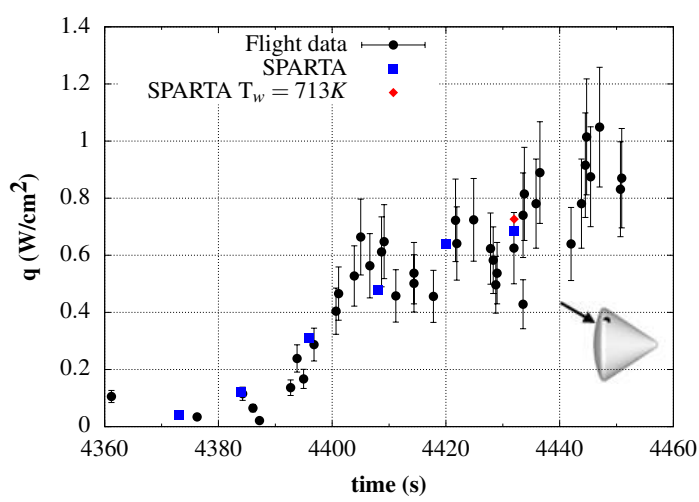

(f) Calorimeter $\mathrm{f}$

Figure 19: Comparison of DSMC and flight data of the AS-202 module. 


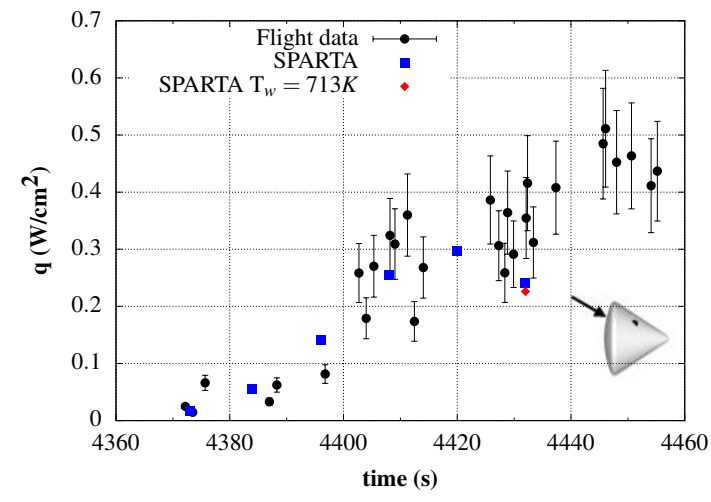

(g) Calorimeter g

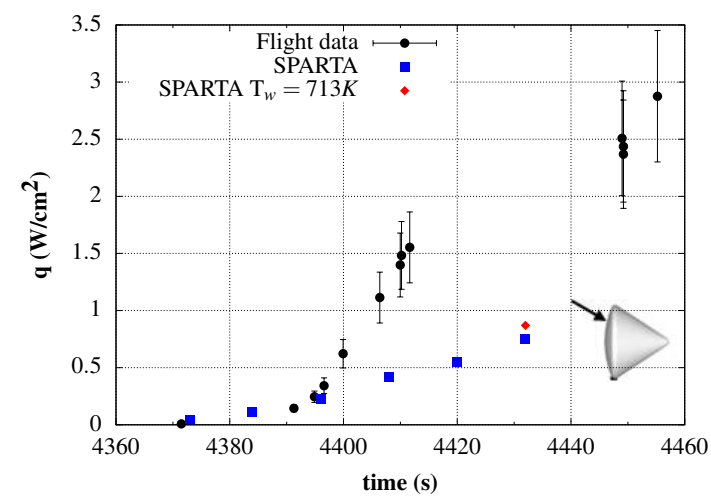

(i) Calorimeter i

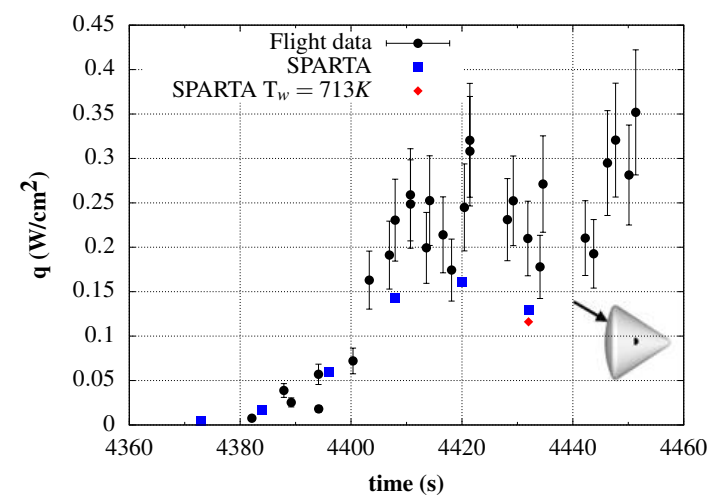

(k) Calorimeter $\mathrm{k}$

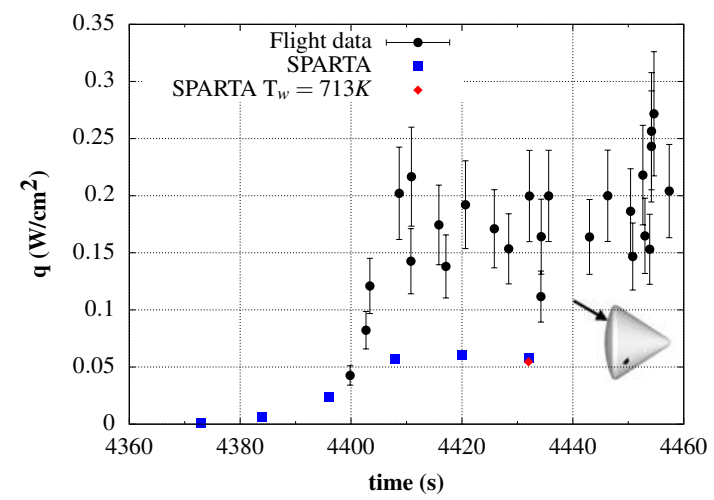

(m) Calorimeter $\mathrm{m}$

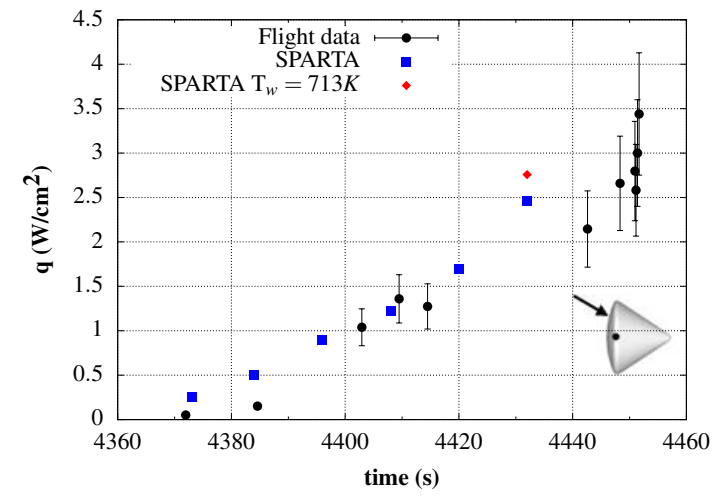

(h) Calorimeter $\mathrm{h}$

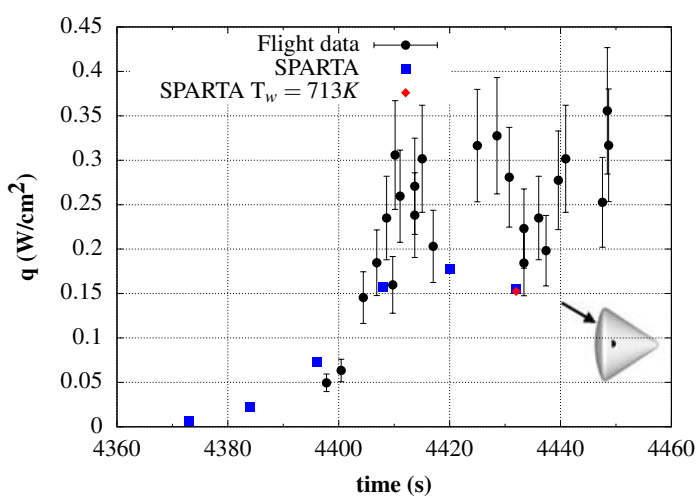

(j) Calorimeter j

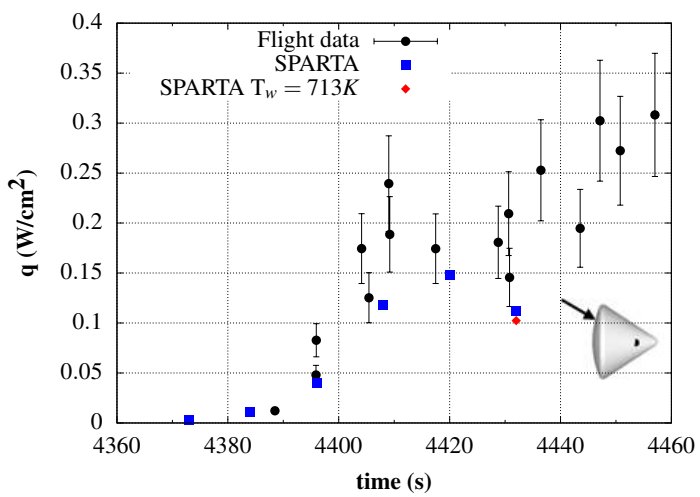

(l) Calorimeter 1

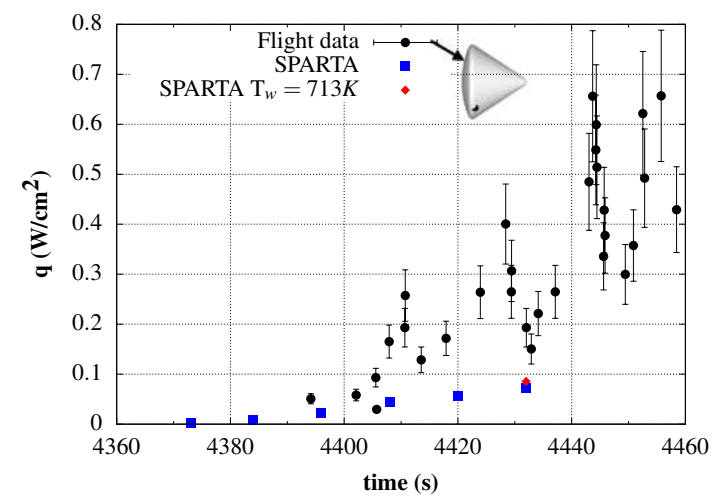

(n) Calorimeter $\mathrm{n}$

Figure 19: Comparison of DSMC results and flight data of the AS-202 module (continued). 


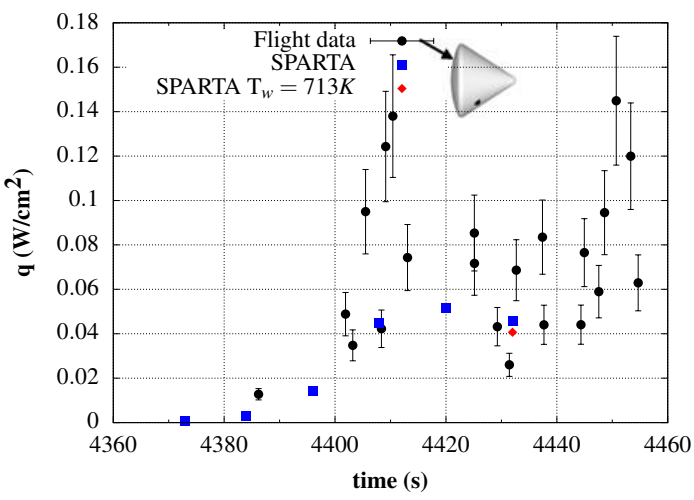

(o) Calorimeter o

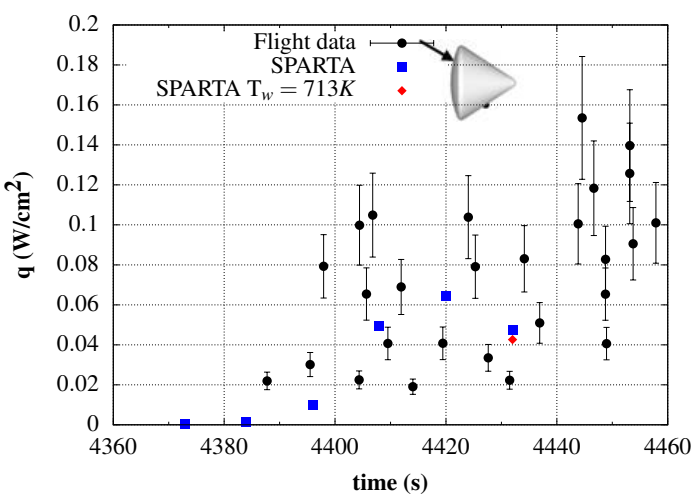

(q) Calorimeter q

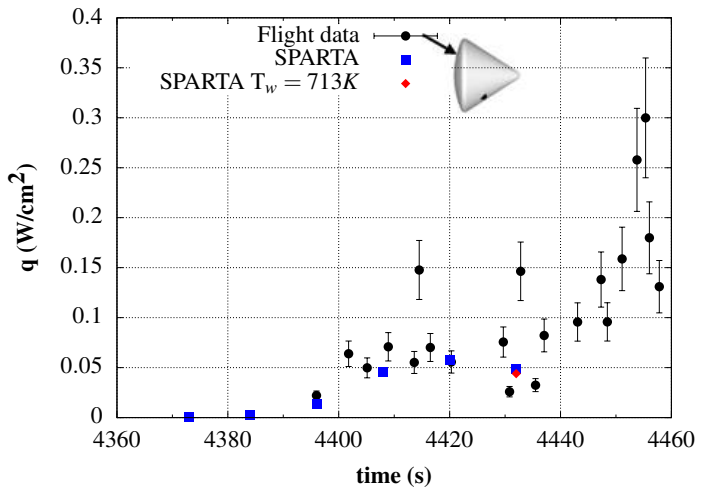

(p) Calorimeter $\mathrm{p}$

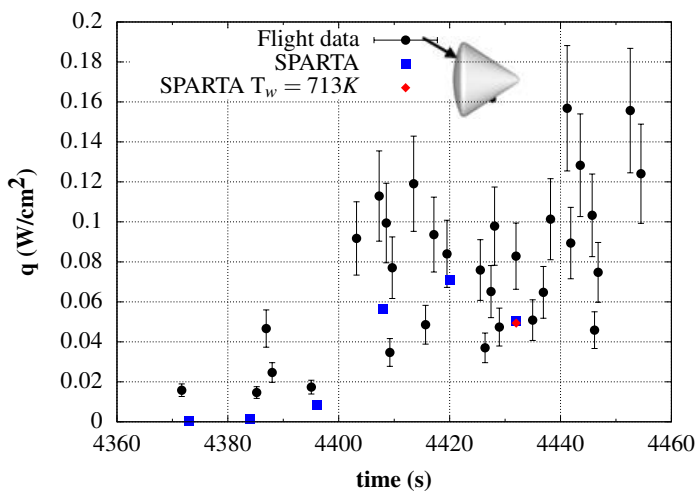

(r) Calorimeter $\mathrm{r}$

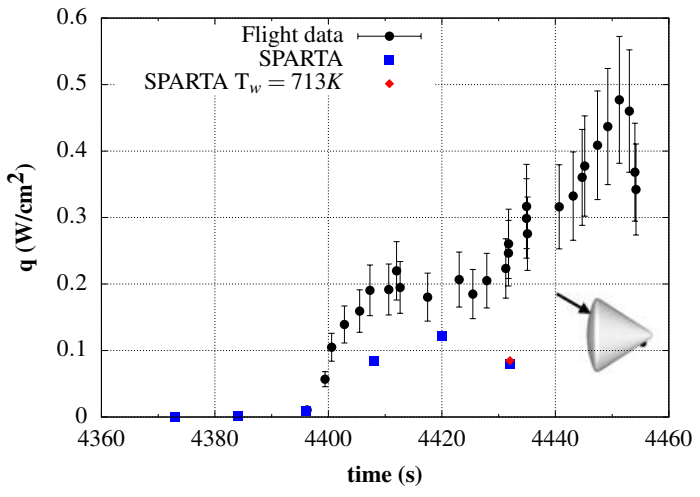

(s) Calorimeter $\mathrm{s}$

Figure 19: Comparison of DSMC results and flight data of the AS-202 module (end).

\subsection{The Space Shuttle Orbiter}

The Space Transportation System (STS) also known as the Space Shuttle was the first partially reusable spacecraft which flew from 1981 until 2011. The space shuttle fleet was composed of five shuttle systems: Columbia, Challenger, Discovery, Atlantis and Endeavour. With this fleet, a total of 135 missions were achieved for various tasks such as the ISS construction, the conduct of in orbit scientific experiments and the launch of satellites. The Shuttle was composed of the Orbiter itself, an external tank and solid rocket boosters (Figure 20a) and was capable of transporting payload from and to Low Earth Orbit (LEO). The shuttle was thus able to perform complex on-orbit operations such as rendezvous, docking before de-orbiting and make precise landings [60] (Figure 20b).

The shuttle was envisaged as a vehicle that would behave like a spacecraft during the first portion of entry and that would fly as an aircraft during the final phase of entry. Thus, the unique nature of this vehicle posed an unprecedented aerothermodynamic challenge. Indeed, the vehicle as envisioned was statically unstable for a period of its operation mode and the 


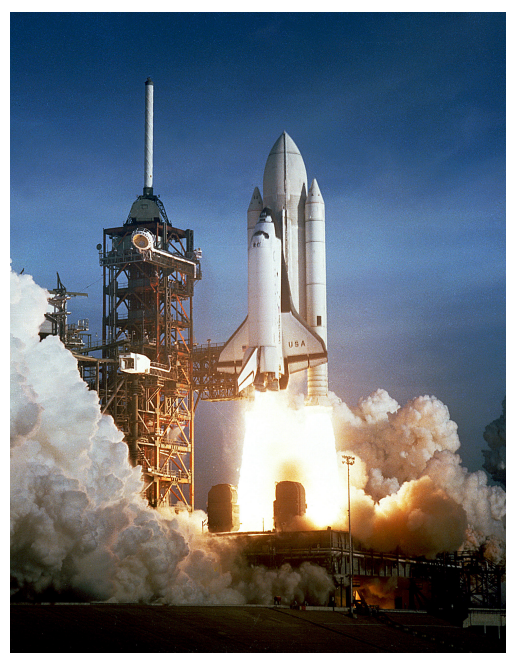

(a) STS-1 launch on April 12, 1981 (credits: NASA).

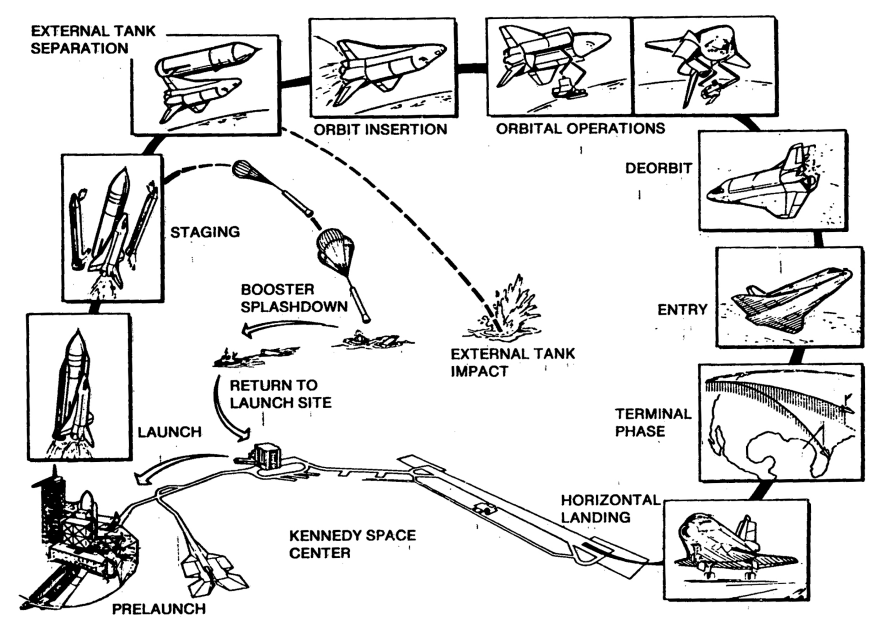

(b) Space Shuttle nominal mission phases (from [61]).

Figure 20: STS launch and operative phases.

accuracy required by manned flight implied the development of precise preflight aerodynamic predictions [62]. The shuttle TPS design relied mostly on wind-tunnel test data which were then evaluated with flight test data [63]. Finally, the TPS was made of reinforced carboncarbon and reusable surface insulation which were chosen for high temperature and weight efficiency [64].

The shuttle orbiter's entry presents complex phenomena involving nonequilibrium effects with air dissociation and catalytic surface recombination. A lot of effort were carried out in order to accurately simulate such effects on the flowfield structure and on the surface heating [65]. In this respect, the shuttle's flight data measured by the Development Flight Investigation (DFI) were thoroughly investigated [66]. The DFI consisted in thermocouples located within the TPS, at around 200 locations. These TCs measured temperature histories that were then used in an inverse 1D, transient heat-transfer analysis to determine convective heat fluxes on the surface [65]. The shuttle was also carrying an orthogonal triaxial set of sensitive linear accelerometers used for the High Resolution Accelerometer Package (HiRAP) experiment. The objective of this experiment was to provide accurate measurements of lowlevel aerodynamic accelerations occurring in the rarefied flow regime along the shuttle's principal axes [67], [68], [69].

The space shuttle is a very rich study case since both aerodynamic and heating rate flight data are available. Since the shuttle's geometry is much more complex than those mentioned in the present paper, getting access to a reliable design can be complicated. However, for NASA related work, several authors performed DSMC simulations of the shuttle orbiter's entry for comparison purposes and DSMC was even used in support of the STS-107 accident investigation [70].

Moss and Bird [71] investigated heat fluxes applied to the STS and only simulated the nose region of the shuttle between 92 and $150 \mathrm{~km}$ in the conditions given in Table 10 . For the calculations, a hyperboloid of nose radius $R_{N}$ and body half angle $\theta$ was used as an equivalent axisymmetric body. As explained in Shinn's paper [65], the concept of such an equivalent axisymmetric body was introduced by Adams et al. [72] who obtained a good agreement between computed and experimental heat transfer data on the windwardray of the shuttle at 30 degrees AoA. The same concept was further verified by Zoby [73] over a range of AoA varying from 25 to 45 degrees. The parameters $\theta$ and $R_{N}$ of the simplified geometry used by Moss and Bird are given in [71]. According to their paper, the 


\begin{tabular}{cccccccc}
\hline \hline Altitude $(\mathrm{km})$ & $\rho_{\infty}\left(\mathrm{kg} / \mathrm{m}^{3}\right)$ & $U_{\infty}(\mathrm{km} / \mathrm{s})$ & $T_{\infty}(\mathrm{K})$ & $X_{\mathrm{O}_{2}}$ & $X_{\mathrm{N}_{2}}$ & $X_{\mathrm{O}}$ & $K n$ \\
\hline 92.35 & $2.184 \times 10^{-6}$ & 7.50 & 180 & 0.217 & 0.783 & 0 & 0.028 \\
99.49 & $5 . .906 \times 10^{-7}$ & 7.50 & 190 & 0.217 & 0.783 & 0 & 0.098 \\
104.93 & $2.457 \times 10^{-7}$ & 7.47 & 223 & 0.153 & 0.782 & 0.065 & 0.23 \\
109.75 & $1.146 \times 10^{-7}$ & 7.47 & 249 & 0.123 & 0.771 & 0.106 & 0.48 \\
115 & $4.380 \times 10^{-8}$ & 7.50 & 304 & 0.098 & 0.754 & 0.148 & 1.22 \\
122.5 & $1.790 \times 10^{-8}$ & 7.50 & 401 & 0.080 & 0.723 & 0.197 & 2.91 \\
130 & $8.230 \times 10^{-9}$ & 7.50 & 500 & 0.071 & 0.691 & 0.238 & 6.20 \\
150 & $2.140 \times 10^{-9}$ & 7.50 & 733 & 0.055 & 0.615 & 0.330 & 22.7 \\
\hline \hline
\end{tabular}

Table 10: Freestream conditions for the STS flight simulations (adapted from [71]).

VHS model was used with the Larsen-Borgnakke phenomenological model [13] for energy exchanges between the translational and internal modes. For the simulation of chemical reactions, Bird's TCE model is employed with a 5-species air model and 34 reactions. The DSMC results are compared with flight data and continuum predictions [74]. The sensitivity to the gas-surface interaction model (full thermal accommodation and $50 \%$ specular) as well as surface catalysis are also investigated in [71] and discussed below.

The heat-transfer coefficient as a function of the Knudsen number is given in Figure 21a. The results correspond to the position $x / L=0.025$ where $x$ is the distance from the nose to the orbiter and $L=32.9 \mathrm{~m}$ is the distance between the nose and the hinge line. With respect to the hyperboloid location $x$, this position corresponds to $0.2 \mathrm{~m}$. The comparison between the DSMC results and the flight data show a good agreement at 92.35 $\mathrm{km}$. However, the differences between the results increase with altitude. Disparities are also visible in Figure 21b which compares flight data and DSMC simulations along the windward centerline in terms of the hyperboloid location $x$, at $110 \mathrm{~km}$. Even if the gap between the numerical results and the flight data decreases with $x$, it still remains significant. Possible explanations of such differences suggested by Moss and Bird are the mass addition to the flowfield due to outgassing phenomena that would reduce the heating and a non-full thermal accommodation. Contrary to the former, the latter source of possible disagreement was investigated and simulations with $50 \%$ specular reflections were made. These simulations showed a substantial change that reduces the differences but as stated by the authors, the agreement cannot be totally reached for realistic accommodation coefficients.

Bird [75] and Rault [76] performed DSMC computations of the STS with complex 3D boundaries/grid generation described in each paper. In both works, the aerodynamic of the shuttle and the flow features are investigated from 170 to $120 \mathrm{~km}$ for Bird and from 170 to $100 \mathrm{~km}$ for Rault. No explicit details are given concerning the DSMC models used by Bird but as for Rault, the DSMC simulations are based on the F3-code and the DSMC models are discussed in details in [76].

The lift to drag ratio (L/D) computed by Bird between 170 and $120 \mathrm{~km}$ are compared with Blanchard's HiRAP experiment in Figure 22. The figure shows that between 130 and $150 \mathrm{~km}$, the DSMC results follow the band's trend. However, as explained by Bird, the value at $120 \mathrm{~km}$ reaches the lower limit of the flight data band. This result is thought to be due to the grid size which does not respect the DSMC accuracy requirements. Rault's simulations are more recent and the computations were pushed down to $100 \mathrm{~km}$ in the atmospheric conditions and with the grid discretization described in [76]. The results are given in Figure 22 and a very good agreement is observed with the flight data for all simulated altitudes. Indeed, the figure shows that Rault managed to overcome Bird's discretization limitation and obtained results in good agreement with the HiRAP experiment down to $100 \mathrm{~km}$. Finally, 
the differences between the wind tunnel and the flight data illustrated in Figure 22 show that the wind tunnel experiments did not manage to exactly retrieve the rarefied conditions encountered by the STS. This incapacity of perfect-gas wind tunnel tests to capture flight aerodynamics was established in the early 90s [77], [78].

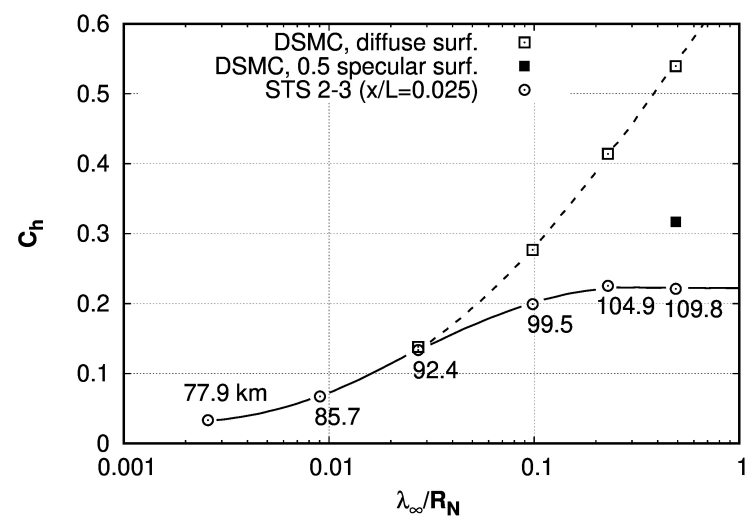

(a) Heat transfer coefficient versus Knudsen number at $x / L=0.025$ (extracted from [71]).

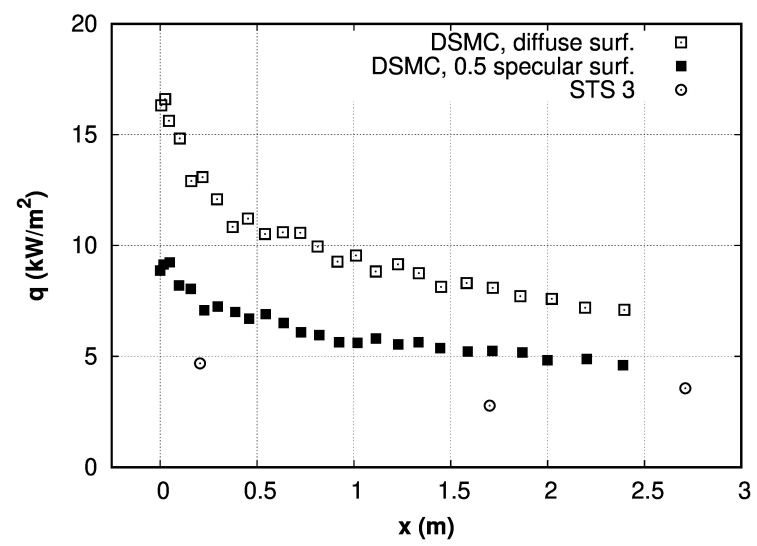

(b) Heat transfer distribution along the windward centerline at $109.75 \mathrm{~km}$ (extracted from [71]).

Figure 21: DSMC and Flight STS heating results.

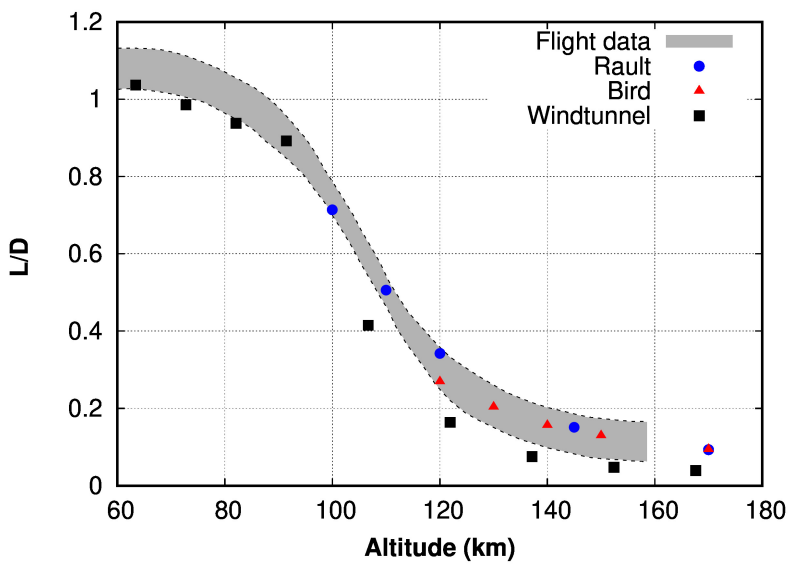

Figure 22: Aerodynamic DSMC results [75], [76] versus experimental [79] and flight data [80].

The simulations presented in this section show how the evolution of computational resources enabled to perform more and more complex and accurate simulations. Since the last results, computational resources have kept growing and DSMC simulations of the complete Shuttle geometry could now be performed below $100 \mathrm{~km}$.

\subsection{The Orbital Reentry Experiment - OREX}

The Japanese Orbital Reentry Experiment (OREX) vehicle was launched the $4^{\text {th }}$ of February 1994 with an H-II rocket, then executed a deorbit maneuver and performed a ballistic flight during the reentry phase [81]. It was the first experiment of a three flight series in preparation of Japan's space program HOPE whose two objectives were to gather flight data and test the TPS developed for HOPE [82]. The vehicle consisted of a $50^{\circ}$ spherically blunted cone with a $1.35 \mathrm{~m}$ nose radius and a base diameter of $3.4 \mathrm{~m}$ (Figure 23). Aerothermal data were collected between 120 and $40 \mathrm{~km}$ and the sensors locations on the vehicle's forebody are given in [82]. The vehicle's TPS was mainly made of a carboncarbon protective layer, an aluminum honeycomb shell and ceramic tiles [82]. 
Gupta, Moss and Price published their work on the OREX reentry in two papers. The first paper [82] presents the results of a viscosity shock layer (VSL) analysis of the reentry flowfield around the forebody of the vehicle between 105 and $48.4 \mathrm{~km}$. The results are compared with flight data; for high altitudes (superior to $80 \mathrm{~km}$ ), the results are compared with DSMC results. These ones are the subject of the second paper [83]. Calculations between 80 and $200 \mathrm{~km}$ are presented and compared with measured values such as axial acceleration, surface pressure and stagnation point heating. 2D/axisymmetric DSMC simulations were performed using the VHS collision model, a 5-species air model. The vibrational and rotational mode were activated and energy exchanges were performed with the Larsen-Borgnakke model [13]. Rotational and vibrational constant collision number were taken with values of respectively 5 and 50. For the calculations, the gas-surface interaction model was assumed diffuse with full thermal accommodation and finite catalytic. For altitudes over $105 \mathrm{~km}$, the wall temperature was assumed constant $T_{w}=331.8 \mathrm{~K}$ while for altitudes less than $105 \mathrm{~km}$, Navier-Stokes solutions coupled with a material response code referenced in [83] were used to obtain the surface temperature distributions. The atmospheric conditions and DSMC results are tabulated in [83].

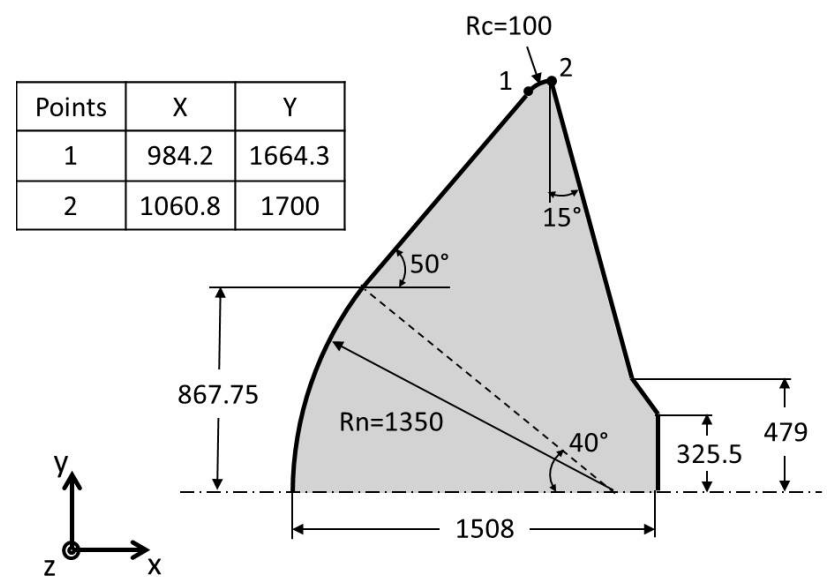

Figure 23: Schematic of OREX configuration (adapted from [83]). Dimensions in mm.

The stagnation-point heating results are given in Figures 24a and 24b. The heating rates are inferred from the temperature measurements made on the back of the surface. For the heat transfer coefficient $\left(C_{h}\right)$, the general trends of the DSMC calculations and inferred flight data are consistent but differences up to $35 \%$ are observable at around $100 \mathrm{~km}$. The authors state that the finite catalytic and non-catalytic boundary conditions give same results for altitudes between 105 and $79.9 \mathrm{~km}$ which means that the wall catalycity is not responsible for the discrepancies. In order to investigate the disagreement for the three critical altitude (Table 11), we performed 3D simulations with SPARTA with the numerical parameters given in Table 12. The wall temperature was taken constant, the vibrational collision number was calculated with the Millikan-White formula and two accommodation coefficients of respectively 1 and 0.8 were tested. The others parameters were taken accordingly to Moss' calculations. The results are given in Figure 24b. For the fully diffuse boundary condition, similar DSMC results were obtained. With an accommodation coefficient $w=0.8$, the difference with the flight data is reduced of approximately $10 \%$ for the 105 and $101 \mathrm{~km}$ altitudes. However, the change in the heat flux for the $96.8 \mathrm{~km}$ is too small to be noticed on the $C_{h}$ graph. It is then concluded that the accommodation coefficient, and thus gas-surface interactions, play a significant role at some altitudes; here altitudes greater than $100 \mathrm{~km}$.

During the vehicle's reentry, two pressure measurements were made with systems described in [83]. For altitudes smaller than $95 \mathrm{~km}$, the DSMC surface pressure agrees well 


\begin{tabular}{ccccccccc}
\hline \hline Alt. $(\mathrm{km})$ & $U_{\infty}(\mathrm{m} / \mathrm{s})$ & $n_{\infty}\left(\cdot / \mathrm{m}^{3}\right)$ & $T_{\infty}(\mathrm{K})$ & $T_{w} \mathrm{a}(\mathrm{K})$ & $K n$ & $\mathrm{X}_{\mathrm{O}_{2}}$ & $\mathrm{X}_{\mathrm{N}_{2}}$ & $\mathrm{X}_{\mathrm{O}}$ \\
\hline 105 & 7451 & $5.0515 \times 10^{18}$ & 211 & 331.8 & 0.0718 & 0.1528 & 0.7815 & 0.0657 \\
101.1 & 7454.6 & $1.0326 \times 10^{19}$ & 196.9 & 401.5 & 0.0346 & 0.1726 & 0.7839 & 0.0435 \\
96.8 & 7456.3 & $1.9812 \times 10^{19}$ & 190.3 & 485.2 & 0.0179 & 0.1884 & 0.7863 & 0.0253 \\
\hline \hline
\end{tabular}

${ }^{a}$ Stagnation temperatures from CFD calculations for altitudes of 105 and less.

Table 11: Freestream conditions for the OREX simulations (adapted from [83]).

\begin{tabular}{ccccc}
\hline \hline Altitude $(\mathrm{km})$ & $\lambda_{\infty} / \Delta_{\mathrm{x}, \mathrm{y}, \mathrm{z}}$ & Adaptive refinement & Number of particles & $\tau_{\text {coll }} / \Delta t$ \\
\hline 105 & 4.8 & Yes & $450 \times 10^{6}$ & 3.6 \\
101.1 & 3.4 & Yes & $530 \times 10^{6}$ & 3 \\
96.8 & 3 & Yes & $590 \times 10^{6}$ & 2.6 \\
\hline \hline
\end{tabular}

Table 12: Numerical parameters for the SPARTA simulations of the critical flight points of the OREX trajectory.

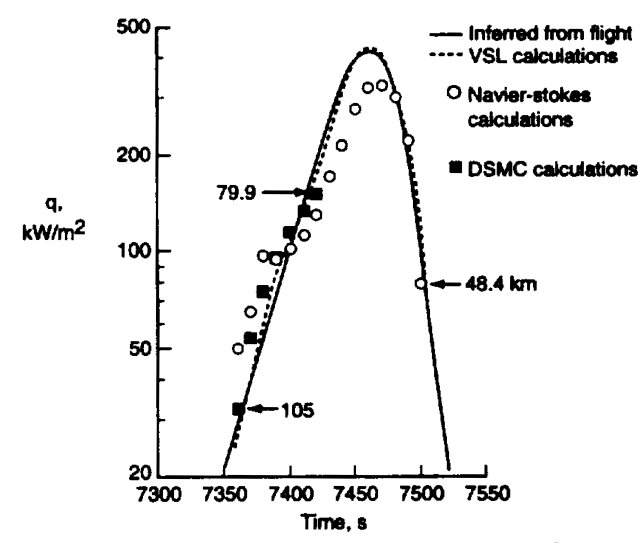

(a) Heating rates (from [83])

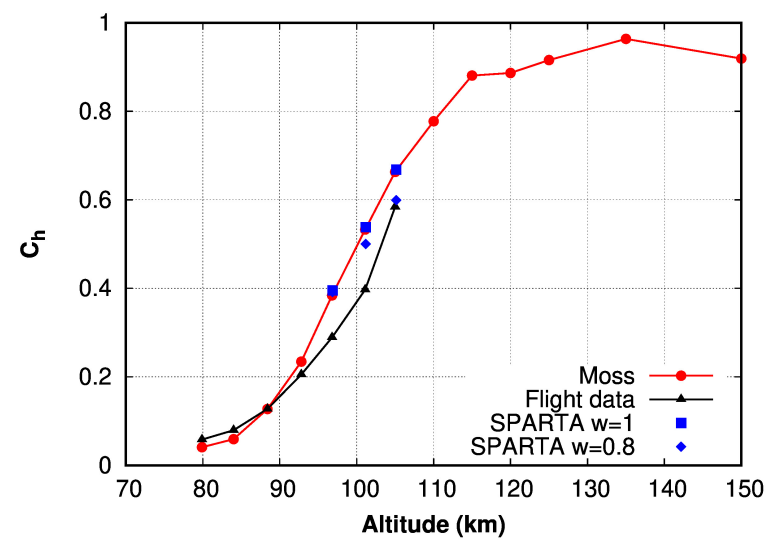

(b) Heating rate coefficient

Figure 24: Comparison of the measured stagnation-point heating with DSMC results for OREX (from [83]).

with the measurements but the results diverge with increasing altitudes for altitudes greater than $95 \mathrm{~km}$ (Figure 25a). According to the results of Moss and Bird [84], this behavior is due to the highly nonequilibrium state at the orifice inlet and along the tube connection to the pressure transducer. Finally, the acceleration was computed from the drag coefficient for an entry mass assumed constant at a value of $761 \mathrm{~kg}$ and a cross-sectional area of 9.0792 $\mathrm{m}^{2}$. An excellent agreement is found with the flight data (Figure 25b).

In conclusion, the DSMC and flight data present a good correspondence overall which is encouraging. Moreover, for most of the transitional flow regime, the heat transfer flight data exhibit a behavior characteristic of a non-blowing surface (unlike the STS data) which makes these data unique. Moss et al. [83] concluded their paper expecting further heating rate data however, to our knowledge, no complementary flight and computational results of the OREX vehicle in rarefied regime were published since then.

\subsection{MIRKA}

MIRKA is a $1 \mathrm{~m}$ diameter sphere shaped capsule launched in October 1997 by a Russian SOJUS rocket and FOTON capsule. It successfully reentered Earth's atmosphere 14 days later along a ballistic trajectory. This German coordinated project had both aerothermodynamic measurement and heat-shield testing objectives. The heat-shield is made of a surface protected ablator covered in a thin layer of $\mathrm{C} / \mathrm{SiC}$ which preserves the spherical shape even 


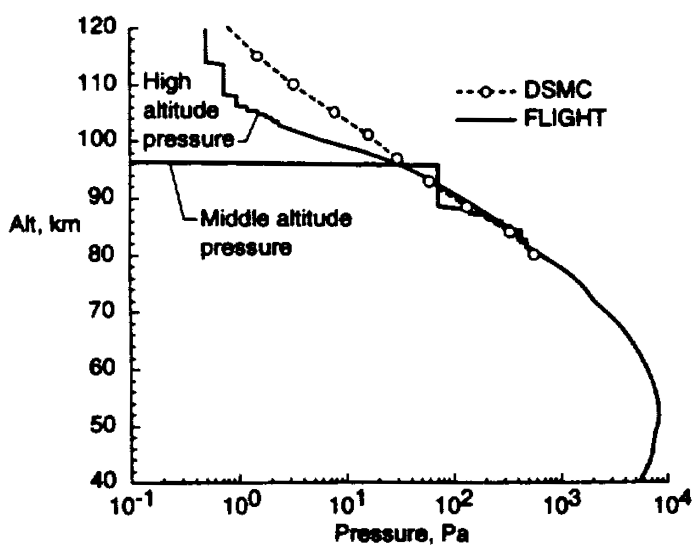

(a) Comparison of numerical and measured pressures on forebody cone (from [83]).

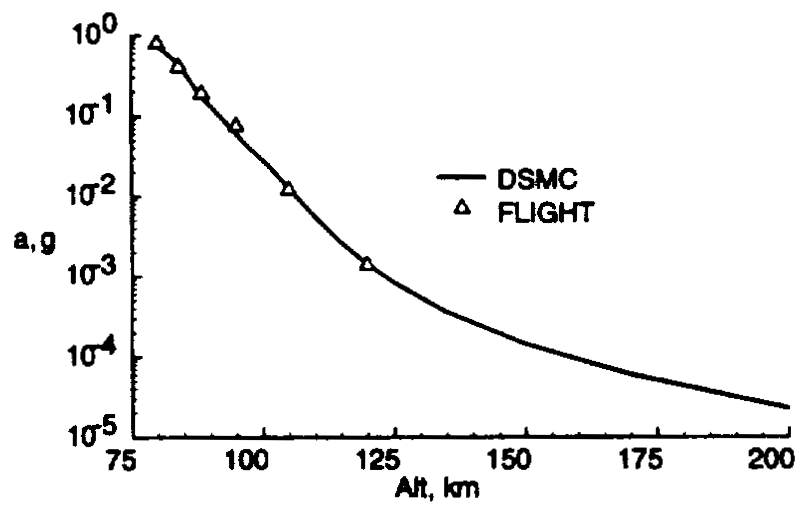

(b) Comparison of DSMC and flight acceleration (from $[83]$ ).

Figure 25: Comparison of the measured pressure and acceleration with DSMC results for OREX (from [83]).

as the ablator is melting. MIRKA was carrying three inboard experiments. The HEATIN experiment was designed for the measurement of temperatures inside the ablator. Back temperatures were measured with the PYREX experiment and the RAFLEX II was conceived to measure pressure and heat flux at different positions (Figure 26) [85].

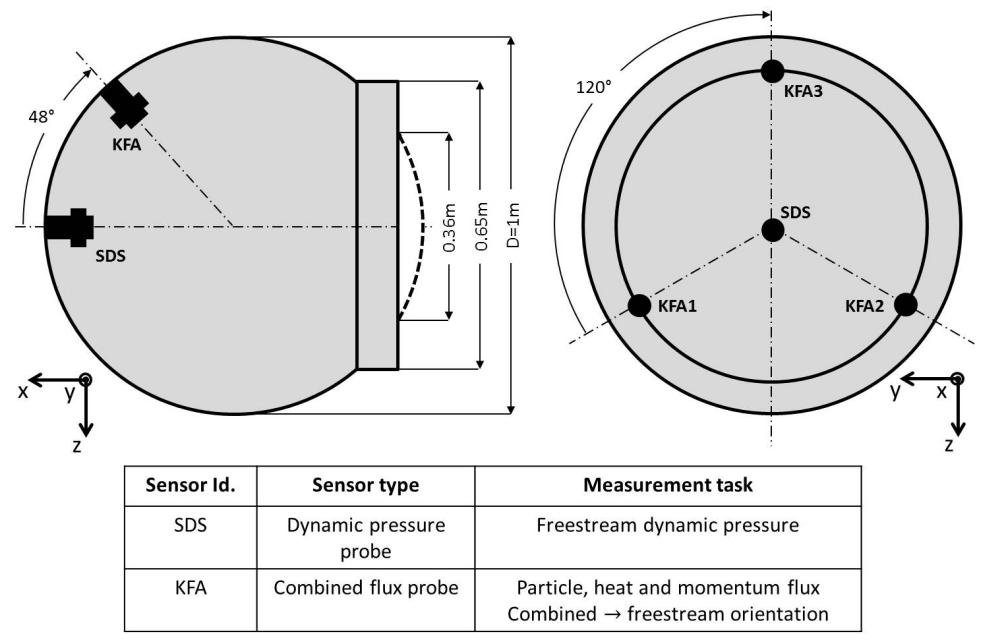

Figure 26: MIRKA geometry and RAFLEX instrumentation (adapted from [85]).

Several simulations were performed on this case [86], [87], [88] but none of them dealt with the rarefied portion of the reentry. In this paragraph, the aim is to draw the attention to Müller-Eigner et al. work [85] which presents and describes the RAFLEX aerothermodynamic data. In this reference, convective heat fluxes measured by the probes are given. Values of about $500 \mathrm{~kW} / \mathrm{m}^{2}$ are found for altitudes around $80 \mathrm{~km}$, corresponding to $K n=3.8 \times 10^{-3}$. This leads to believe that significant heat fluxes are applied to the capsule during the rarefied portion of its reentry. The investigation of these results could then be a significant source of learnings. However, even if numerical trajectory reconstruction data are available in [85], the capsule was not carrying onboard IMU. This means that no precise trajectory data are available which might complicate the MIRKA reentry analysis [3].

\section{Ionization and radiative flight data}

High velocities of an order of magnitude of $10 \mathrm{~km} / \mathrm{s}$ are characteristic of superorbital reentry. In this context, the gas is partially ionized and the electronic mode gets excited. For 
such electronic excitation, thermal radiation becomes significant [89] and must be taken into account. The radiative heat flux can either be calculated directly inside the DSMC code or it can be calculated by the coupling with a radiation code. Another considerable mechanism happening when the gas is ionized is the densification of the electron population that can lead to communication blackout and prevent the vehicle from exchanging information with the control stations. These kind of phenomena can happen at altitudes high enough for nonequilibrium and rarefaction effects to be important. Therefore, DSMC modeling can be required. Several reentry flights were designed to collect flight data related to ionization and thermal radiation. The data comparison to DSMC simulations is the object of this part.

\subsection{Fire $I I$}

Project Fire was a NASA research program in preparation for the Apollo Lunar journey. The project aimed at collecting heating data in order to have a better understanding of the convective and radiative environment associated to superorbital return. Two flights took place in April 1964 for Fire I and May 1965 for Fire II. Both flights followed a ballistic trajectory with entry parameters given in Table 13. The Fire probe was an Apollo-like shaped

\begin{tabular}{ccccc}
\hline \hline Flight Id. & Launch date & Velocity $(\mathrm{km} / \mathrm{s})$ & $\alpha($ deg.) & $\gamma($ deg.) \\
\hline Fire-I & April 14, 1964 & 11.56 & 0 & -14.7 \\
Fire-II & May 22, 1965 & 11.35 & 0 & -14.7 \\
\hline \hline
\end{tabular}

Table 13: Launch conditions of Fire flight tests [90].

object whose afterbody was instrumented with three spherical-section beryllium calorimeters, two radiometers and a spectrometer package for shock layer radiation measurements. The afterbody was equipped with nine surface-mounted TCs, one pressure sensor and a single radiometer [3]. The geometry is represented in Figure 27. The forebody instrumen-

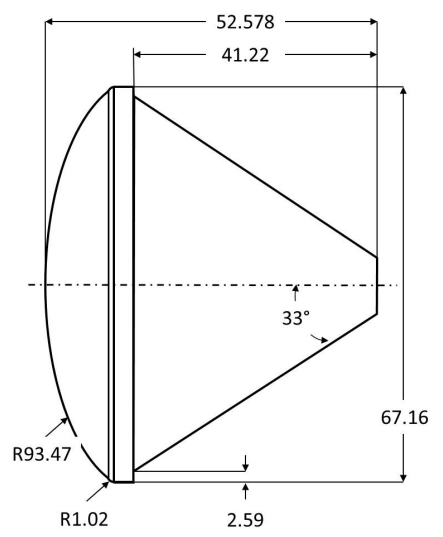

Figure 27: Simplified Fire II geometry (adapted from [91]). Dimensions in cm.

tation was backed by phenolic-asbestos heat shields and the afterbody TPS was made of an ablative silica-phenolic material [90].

As detailed in [92], the Fire I probe underwent significant angle of attack oscillations which ultimately lead to a difficult interpretation of the measured data [3]. Therefore, the analysis of the Fire project data focuses on the Fire II probe whose entry was highly successful [90].

In 1987, Bird [89] investigated ionization and thermal radiation with a 1D DSMC program capable of simulating 1D flow along a stagnation streamline. This program used a VHS model for the intermolecular collisions and the Larsen-Borgnakke model for energy 
exchanges with the rotational and vibrational modes and constant collision numbers of respectively 5 and 50 were used. The addition of electrons and charged species resulted in a 11-species air model with a set of 41 chemical reactions modeled with the TCE relaxation procedure. The ambipolar approximation assumption is employed. This approximation conserves neutral charge by constraining the motion of each electron to follow that of its associate ion with which it was created. As a consequence, the average motion of the electrons is affected without changing their thermal velocities. A phenomenological model similar to Larsen-Borgnakke described in [89] was used to take into account thermal radiation and energy exchanges with the electronic mode. Sensitivity to surface catalycity and thermal accommodation coefficients were also studied. The flight conditions of the two simulated altitudes are given in Table 14 .

The radiometer measured stagnation point radiative heating rate for wavelength between $0.2 \mu \mathrm{m}$ and $4.0 \mu \mathrm{m}$ and calorimeters measured total heating rate. The comparison of the DSMC results with the flight data for the heat flux to the stagnation point are given in Table 15 where $q_{\mathrm{rad}}^{\text {exp. }}$ and $q_{\mathrm{rad}}^{\mathrm{DSMC}}$ are the experimental and DSMC radiative heat flux for wavelengths of $0.2 \mu \mathrm{m}$ and above.

\begin{tabular}{ccccc}
\hline \hline Altitude $(\mathrm{km})$ & $U_{\infty}(\mathrm{km} / \mathrm{s})$ & $n_{\infty}\left(\cdot / \mathrm{m}^{3}\right)$ & $T_{\infty}(\mathrm{K})$ & $K n$ \\
\hline 75 & 11.31 & $7.94 \times 10^{20}$ & 198 & $2.5 \times 10^{-3}$ \\
60 & 11.03 & $6.87 \times 10^{21}$ & 253 & $3 \times 10^{-4}$ \\
\hline \hline
\end{tabular}

Table 14: Freestream conditions for the Fire II simulations (adapted from [89]).

\begin{tabular}{ccccc}
\hline \hline Alt. $(\mathrm{km})$ & $q_{\mathrm{rad}}^{\text {exp. }}\left(\mathrm{kW} / \mathrm{cm}^{2}\right)$ & $q_{\mathrm{rad}}^{\mathrm{DSMC}}\left(\mathrm{kW} / \mathrm{cm}^{2}\right)$ & $q_{\mathrm{conv}}^{\text {exp }}\left(\mathrm{kW} / \mathrm{cm}^{2}\right)$ & $q_{\mathrm{conv}}^{\mathrm{DSMC}}\left(\mathrm{kW} / \mathrm{cm}^{2}\right)$ \\
\hline 75 & 0.01 & 0.011 & 0.18 & 0.19 \\
60 & 0.16 & 0.14 & 0.7 & 0.72 \\
\hline \hline
\end{tabular}

Table 15: Fire II flight measurements and computed heat fluxes at stagnation point with a 1D approach (adapted from [89]).

According to Bird, the results are very satisfactory because agreement is attainable for reasonable choices of surface properties. However, Bird precises that complete sensitivity analysis of the data values were not accessible with personal computers which is why the employed data could only be considered as preliminary estimations.

With the computational capacity increase, Bird and Moss [93] extended the method from $1 \mathrm{D}$ to $2 \mathrm{D}$ axisymmetric. Their results showed that the $1 \mathrm{D}$ method could be deficient namely because the radiation contribution to the surface from particles removed from the computational domain would not be considered. Taylor et al. then proposed another 2D axisymmetric DSMC ionization model [94] that they finally extended to include both ionization and radiation [95]. In [95], the method was evaluated by confrontation with Bird's method with respect to the results of Project Fire II at an altitude of $76.42 \mathrm{~km}$, a velocity of $11.36 \mathrm{~km} / \mathrm{s}$ and a distance $S=0.03 \mathrm{~m}$ from the nose along the vehicle's surface. Three sets of results are discussed. The first two series of results are those obtained with Bird's ionization method without and with radiation included. The last one consist of Taylor's method including both ionization and radiation. For the three sets, the domain was made of 18000 cells adjacent to a portion of the probe's central forebody [95].

Table 16 summarizes the results inferred from Taylor's paper [95]. In this table, $q_{\mathrm{rad}}^{0.2 \mu}$ is the radiative heat flux for wavelengths of $0.2 \mu \mathrm{m}$ and above, and $q_{\mathrm{rad}}^{\mathrm{tot}}$ is the radiative heat flux for all wavelengths. The simulations based on both Bird's methods led to similar convective 
heat flux. For the radiative heat flux, Taylor's new electronic excitation procedure gives a better value when only contributions from $0.2 \mu \mathrm{m}$ and above are considered but the method gives similar radiative heat flux to Bird's method when all wavelengths are considered. Hence, it is very important to be able to correctly model electronic relaxation for a good prediction of radiation and wall heat flux. Moreover, the quality of the results could be fortuitous and only the incorporation of an absorption model could confirm it.

\begin{tabular}{cccc}
\hline \hline Method & $q_{\mathrm{rad}}^{0.2 \mu}\left(\mathrm{W} / \mathrm{cm}^{2}\right)$ & $q_{\mathrm{rad}}^{\text {tot }}\left(\mathrm{W} / \mathrm{cm}^{2}\right)$ & $q_{\mathrm{conv}}\left(\mathrm{W} / \mathrm{cm}^{2}\right)$ \\
\hline Flight data & $8.2 \pm 20 \%$ & - & $172 \pm 5 \%$ \\
Bird without rad. & - & - & 220 \\
Bird with rad. & 88 & 195 & 225 \\
Taylor & 20 & 175 & - \\
\hline \hline
\end{tabular}

Table 16: Fire II flight measurements and computed heat fluxes at $0.03 \mathrm{~m}$ along the vehicle's surface (adapted from [95]).

More recently, Farbar and Boyd [96] presented axisymmetric DSMC simulations about the Fire II with MONACO. Two points along the trajectory are considered: in the noncontinuum region at $85 \mathrm{~km}$ and in near-continuum regime at $76 \mathrm{~km}$. The ambipolar diffusion assumption was used and simulations with 5 and 11 species air were made. The work investigates the sensitivity to molecular interaction model for all the colliding pairs and the vibrational relaxation model for collisions of electrons and nitrogen molecules.

The measured total heat flux is compared to the values calculated by DSMC using the 11-species chemistry. Simulations lead to a heat flux near the upper boarder of the $\pm 5 \%$ uncertainty at an altitude of $85 \mathrm{~km}$. At $76 \mathrm{~km}$, the calculated heat flux including the radiative component coincides with the lower boarder of the $\pm 5 \%$ uncertainty. For both altitudes, the heat fluxes are compared to Taylor's results [94], [95] and a much better agreement is reached for the new simulations.

Liechty [97] presented an updated version of NASA's DSMC code DAC, capable of simulating charge-neutral ionized flows with electronic energy level transitions modeled with the Quantum-Kinetic (Q-K) chemistry model [98] and which includes the electronic energy mode. Comparisons were made between DAC, DS2V, the CFD code LAURA and the Fire II flight test data point $t=1634 \mathrm{~s}$ which corresponds to an altitude of $76.42 \mathrm{~km}$. In this study, the radiative heat transfer were obtained by post-processing the flowfield solutions with the non-equilibrium radiation code HARA described in [97] and [99]. All DSMC simulations used the VHS collision model, an 11-species air with the TCE chemistry model and a fully catalytic wall condition that converted atoms into their associate molecules and charged species into their associate neutral species when they impact the surface. The wall temperature was taken constant at $615 \mathrm{~K}$.

The results are summarized in Table 17 where DAC is the original version of NASA's DSMC code, DAC-E is the updated version taking into account the electronic energy mode and the last column indicates the sum of the convective and fraction of radiative heating that would have been absorbed by the calorimeter on the vehicle. The results of convective heat flux show an improvement with respect to the flight data since the discrepancy decreases from 35\% (DAC) to 20\% (DAC-E). The influence of DAC's modifications are discussed in details in [97]. The difference between the radiative heat flux calculated by DAC-E and the one measured during the flight is very important. One possible reason for such discrepancy is the lack of radiation-flowfield coupling in Liechty's simulations [97]. Indeed, Johnston [100] demonstrated that the radiation-flowfield coupling has almost no effect on the convective heat flux but can lead to a decrease of maximum $30 \%$ in the radiative heat flux. The way 
the non-Boltzmann modeling of the atomic and molecular electronic states is considered constitutes another possible source of error. Recent improvements on that matter were proposed by Panesi in [101].

\begin{tabular}{cccc}
\hline \hline Method & $q_{\text {conv }}\left(\mathrm{W} / \mathrm{cm}^{2}\right)$ & $q_{\text {rad }}\left(\mathrm{W} / \mathrm{cm}^{2}\right)$ & $q_{\text {conv }}+k q_{\mathrm{rad}}\left(\mathrm{W} / \mathrm{cm}^{2}\right)$ \\
\hline LAURA & 217 & 25 & 235 \\
DS2V & 220 & - & - \\
DAC & 220 & - & - \\
DAC-E & 195 & 52 & 232 \\
Flight data & 162 & 18 & 175 \\
\hline \hline
\end{tabular}

The calorimeter absorbed fraction of radiative heating $k=0.72$

Table 17: Fire II flight measurements and computed heat fluxes at $0.1 \mathrm{~m}$ from the symmetry axis (adapted from [97]).

Fire II probably constitutes the most investigated case of study that deals with ionized flows and thermal radiation. The results presented in this section have shown that a proper reconstruction of the heating data is still a challenging task.

\section{2. $R A M C-I I$}

The Radio Attenuation Measurement (RAM) experiments comprised several flight experiments at medium velocity designed to measure plasma parameters around a $9^{\circ}$ hemispherecone with a nose radius $R_{N}=0.1524 \mathrm{~m}$ and a total length of $1.3 \mathrm{~m}$. The two first flights RAM C-I and RAM C-II launched respectively in October 1967 and August 1968 are presented in [102]. The first flight was conceived to evaluate the effect of water injection as plasma alleviation technique and the second flight discussed hereafter aimed at measuring the electron density time and altitude histories between 90 and $60 \mathrm{~km}$ at different locations along the vehicle. For this purpose, the vehicle was instrumented with microwave reflectometers and electrostatic probes (Figure 28). The TPS was made of a non-ablative beryllium nose cap and a teflon afterbody in order to prevent additional ionization due to surface to occur [103].

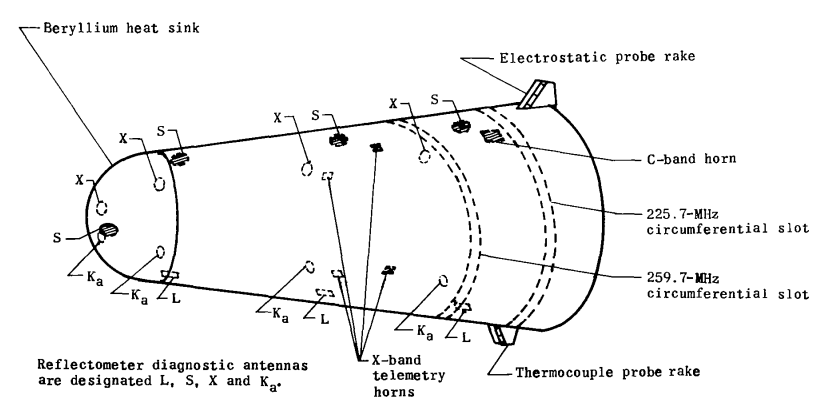

Figure 28: RAM C-II geometry and instrumentation (from [102]).

Although the onset of the communication blackout coincides with altitudes where both surface pressure and heat transfer are in agreement with the values computed from continuum theory, the outer portion of the flowfield is not adequately predicted by continuum theory and nonequilibrium chemical reactions occur. Therefore, non-continuum calculations are required [103]. Because of the mass of electrons which is five order of magnitudes lower than neutral species, the simulation of electrons with DSMC poses three main problems [104]. The first concern is related to the electrons velocity of diffusion which is taken care 
of with the ambipolar approximation assumption discussed in 4.1. The second issue resulting from the electrons mass comes from the fact that the collision probability of a pair of particles is proportional to the relative velocity of this pair. Therefore, when an electron is tested for a collision, its thermal velocity which can be more than two orders of magnitude greater than that of other particles results in a rate of collision significantly higher than for other species. In order to address this problem, the time step can be taken smaller or the collision process can be subdivided [89]. The last problem is a consequence of the ionization reactions whose activation energy at orbital speed result in low reaction probabilities. For a sufficient number of charged particles to be generated, specific numerical schemes must be used.

Bird [103] investigated the ionized flow around the RAM C-II vehicle with the DSMC method. Because the chemical reaction :

$$
\mathrm{N}+\mathrm{O} \rightarrow \mathrm{NO}^{+}+\mathrm{e}^{-},
$$

requires less energy than any other reaction leading to ionization, this reaction only was considered in this work. However, in general, the fraction of ionized particle is around one charged particle for $10^{5}$ neutral particles [103]. With the computational resources of the period, the number of numerical particles of a simulation would be smaller than $10^{5}$ which means that the number of charged particles would be too low to obtain accurate results. Bird consequently addressed this problem by modifying the numerical representativeness of $\mathrm{NO}^{+}$particles [103]. Finally, although the DSMC results obtained by Bird follow the measurements form, the DSMC values are significantly lower than the measured values.

A few years after Bird's attempt to reconstruct the electron density from the RAM CII experiment, Boyd [104] presented new numerical schemes to improve the ability of the DSMC to simulate the plasma layer around a vehicle during its reentry at orbital velocity. The detailed parameters and models described in [104] enabled to address the electrons motion, the low concentration of charged species and the computational cost due to the electron collision rate. This time, three ionization reactions were considered with an 11species reacting gas and specific rotational and vibrational energy exchange models were used. Simulations using the TCE and the Vibrationaly Favored Dissociation (VFD) models for the simulation of dissociation reactions were performed. For compressed hypersonic flows where the thermal equilibrium between the vibrational and the transitional modes of energy is not reached, the vibrational temperature is smaller than the transitional temperature and the VFD model gives smaller dissociation rates. This ultimately results in an inferior production of electrons via the associative ionization reactions [104]. This phenomenon is visible in Figures 29a and 29b which respectively represent the electron number density along the vehicle and across the plasma layer at the electrostatic probe location (Figure 28) referenced in [104] as the Langmuir probe location. For both figures, the electron number density predicted by the VFD model is a factor of 2 inferior to that of the TCE model and the two models globally encompass the flight data.

As concluded by Boyd [104], considering the uncertainty of measurement for such conditions, a very satisfactory level of agreement was obtained between the DSMC results and the two sets of electron number density measurements. This achievement attests the capacity of the DSMC to predict the plasma formed around a hypersonic vehicle. Fang et al. [105] extended Boyd's method to 3D simulations and after validation by comparison to the RAM C-II results, managed to accurately predict the altitudes of communication blackout of the Chinese lunar Capsule.

\subsection{The sample return capsule Stardust}

Stardust was a spacecraft launched by NASA in February 1999 as part of a mission aiming at collecting comet and interstellar dust samples. After successfully collecting those samples, 


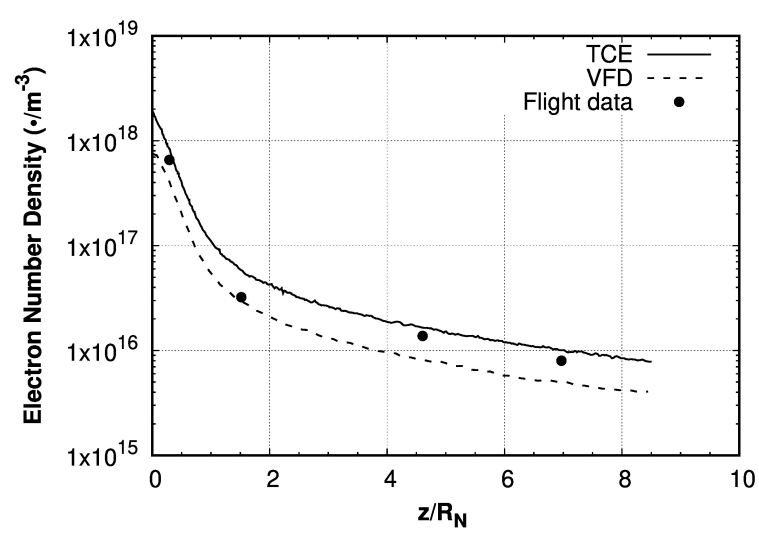

(a) Maximum electron number density along the vehicle.

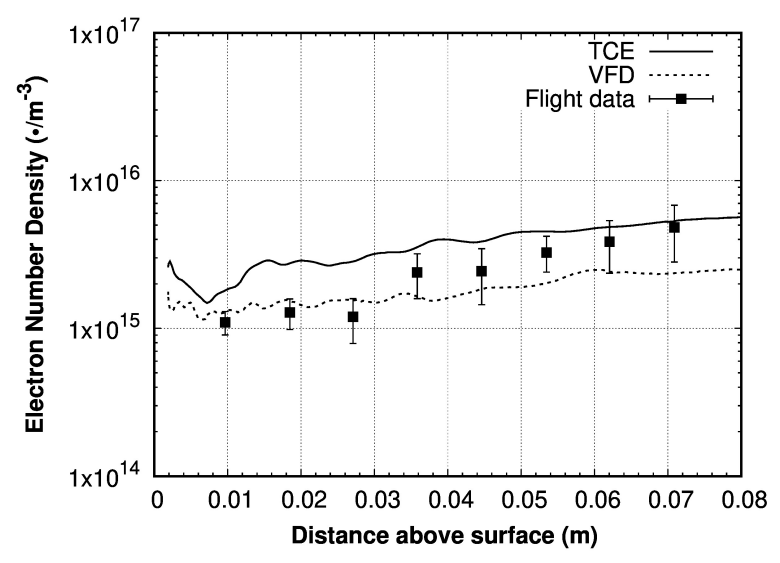

(b) Electron number density at the Langmuir probe location.

Figure 29: Comparison of the measured electron number density with DSMC results at an altitude of 81 km during the RAM-C II Earth entry (extracted from [104]).

they were taken down to Earth with the Sample Return Capsule (SRC) in January 2006. The SRC capsule represented in Figure 30 was protected with a $60^{\circ}$ sphere-cone heat-shield, a truncated conical back-shell and entered Earth's atmosphere at a $12.8 \mathrm{~km} / \mathrm{s}$ velocity [106]. The TPS consisted of a Phenolic-Impregnated Carbon Ablator (PICA) developed by NASA and the SRC was not instrumentated which means that no time-resolved measurements are available. Instead, an auxiliary mission to observe the entry from an airborne platform was conducted [107]. This mission acquired imaging and spectroscopic data for a period

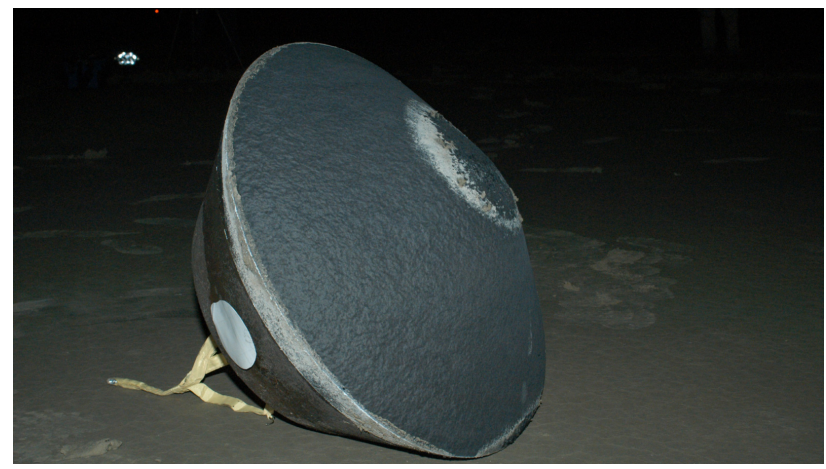

Figure 30: Stardust Sample Return Capsule after landing (credits: NASA).

of $60 \mathrm{~s}$ roughly centered around the peak of heating pulse. Eight instruments were used with different spectral and temporal resolutions for measuring the radiative signals from the shock layer surrounding the capsule [106].

Several numerical simulations were realized in the frame of the SRC's reentry. DSMC, CFD and analytical models were used to study the aerodynamics of the capsule [108], [109]. DSMC simulations were performed for the investigation of electronic excitation and radiation of the SRC flow at $68.9 \mathrm{~km}$ [110]. The aerodynamic heating reduction through the ablation process of the SRC's TPS was studied with the DSMC code SMILE at altitudes of 68.9 and $81 \mathrm{~km}$ [111]. Spectroscopic observations were studied by Jenniskens [112] by means of slitless miniature echelle spectrograph onboard NASA's airborne laboratory. Band emissions of air plasma from the shock layer, atomic and molecular band emissions from the heat-shield and atomic line emissions from the paint on top of the PICA material were captured. Flowfields obtained with the CFD code DPLR were processed with the spectral line-by-line radiation code NEQAIR and compared against the airborne's laboratory data [106]. 
Boyd et al. [113] investigated the SRC's flowfield at 81 and $71 \mathrm{~km}$. The freestream conditions are given in Table 18. In this study, both CFD and DSMC methods were employed to model the flow of the probe's forebody in near-continuum regime. For both methods an 11-species air was used with 19 reactions. The 2D axisymmetric DSMC simulations assumed the surface as fully diffuse at wall temperature indicated in Table 18 and fully catalytic to ions and atoms. No ablation process was considered and chemical reactions were simulated with the TCE and VFD models. Complementary details are available in [113]. In a companion paper [114], Boyd et al. used the flowfield results to perform a radiation analysis with the nonequilibrium radiation code NEQAIR.

\begin{tabular}{cccccccc}
\hline \hline Alt. $(\mathrm{km})$ & $T_{\infty}(\mathrm{K})$ & $n_{\infty}\left(\cdot / \mathrm{m}^{3}\right)$ & $U_{\infty}(\mathrm{km} / \mathrm{s})$ & $X_{\mathrm{O}_{2}}$ & $X_{\mathrm{N}_{2}}$ & $K n$ & $T_{w}(\mathrm{~K})$ \\
\hline 81 & 217.6 & $2.643 \times 10^{20}$ & 12.385 & 23.67 & 76.23 & 0.005 & 2000 \\
71 & 221.6 & $1.156 \times 10^{21}$ & 12.063 & 23.67 & 76.23 & 0.001 & 2700 \\
\hline \hline
\end{tabular}

Table 18: Freestream temperature, number density, velocity, number concentration and Knudsen number as well as wall temperature (adapted from [114]).

In this work [114], both air species and metal species emissions of flux densities $\left(\mathrm{W} / \mathrm{m}^{2} / \mathrm{nm}\right)$ were studied and compared with Jenniskens' data [112]. At $81 \mathrm{~km}$, the DSMC flowfield simulations gave good agreement with the measured spectra of emissions. Analysis of $\mathrm{N}_{2}^{+}$system also led to a good level of agreement between the observation data and the DSMC predictions. However, at $71 \mathrm{~km}$, the DSMC results tended to underpredict the emission spectra. This discrepancy was interpreted as the result of surface blowing phenomena which should be taken into account. Hence, complementary DSMC simulations including fluxes of metal (particles $/ \mathrm{m}^{2} / \mathrm{s}$ ) blowing from the surface at $81 \mathrm{~km}$ were performed. The metal blowing fluxes were adjusted to fit at best the measured line intensities. Then, those blowing fluxes inferred from simulations were compared with the magnitude of the impurity of the material which indicated that some emissions of flux density were due to the white paint and some to the TPS ablation. The mass fractions for blowing from the surface obtained at $81 \mathrm{~km}$ were then used for new simulations at $71 \mathrm{~km}$ and qualitative agreement was obtained with the measured spectra.

Despite the absence of onboard instrumentation, spectroscopic observations enabled the collection of a great amount of radiative signals. State of the art DSMC simulations were performed and inferred line-of sight radiation gave encouraging results compared to measured data for two near-continuum altitudes. Obvious discrepancies are still noticeable which indicates that complementary efforts need to be carried out in the context of ionization and radiation modeling.

Mankodi et al. [115] recently investigated the SRC reentry. In their work, the flow field around the capsule and its surface properties were modeled with DSMC simulations using two different chemical models. The first model is the classic TCE model while the second is an ab initio model based on reactive cross sections calculated with a Quasi Classical Trajectory (QCT) method. Simulations at altitudes of 68, 80 and $100 \mathrm{~km}$ were carried out. Although the results show that the ab initio model predicts higher heat fluxes than the TCE model, the results were not compared to flight data.

\section{Conclusion}

In this survey, experimental, flight and numerical data were presented in the context of hypersonic rarefied flows commonly encountered in the first phase of atmospheric reentry. These results have shown both some capacities and limitations of the DSMC method to simulate rarefied flows that are generally out of the scope of classical CFD methods. 
A large amount of low density experiments were conducted in low density facilities which constitute a solid comparison basis for the validation of DSMC and particle simulation methods. However, the difficulty to retrieve high enthalpy conditions characteristic of atmospheric reentry restricts their use to the study of non-reactive flows. In these conditions, DSMC has proven to be capable of accurately predicting such flows over simple geometries like flat planes with truncated or sharp leading edge. However, the $70^{\circ}$ sphere-cone geometry revealed that for high angles of attack, significant discrepancies were observable between DSMC results and measurements. The non-reactive aspect of the flows produced in lowdensity facilities indicates that improvements are attainable in elementary DSMC models such as translational-rotational energy exchanges and gas-surface interaction models.

Even though the measurement of ATD data in the rarefied portion of the reentry is not the primary objective of flight experiments, high altitude values measured during such flights are sometimes available. Thus, heating rate of Mercury and Apollo AS-202 flight experiments were extracted and compared to DSMC simulations. Another source of unexploited forebody heat flux data inferred from the MIRKA reentry have been addressed. DSMC simulations were performed and compared to aerothermodynamic flight data of other vehicles such as OREX and the Space Shuttle. Given the limited amount of flight data and the uncertainty of the freestram conditions, the numerical results have shown a satisfactory agreement with the measured aerodynamic data. However, the same conclusion cannot be drawn for the aerothermodynamic DSMC results which leave room for significant improvement.

Moreover, the SPARTA simulations showed that a better understanding of the gassurface interaction could lead to better predictions through the role of the accommodation coefficients and the use of a non-uniform wall temperature. These simulations also illustrated the limitations of DSMC in nearcontinuum conditions.

Ionization, thermal radiation and electronic excitation usually happen at low altitudes or at superorbital velocities. However, analysis of various flight experiments involving such phenomena have shown that even at near-continuum altitudes, strong non-equilibrium effects can require the use of particle simulation methods. The last part of this work showed how the various updates of the DSMC method enabled the simulation of complex ionized flows for altitudes lower than $80 \mathrm{~km}$.

Concerning the evolution of the DSMC method, Gimelshein and Wysong outlined in [116] the fact that as for now, the oldest model of chemical reaction is still often the best option. The same paper indicates that only three major improvements from the initial version of the method gave the DMSC its current attractivity. This suggests that most of the old computations presented herein still holds as seen with OREX. Furthermore, current efforts in the context of DSMC improvement are made in several directions. For example, stateto-state energy exchange procedures and chemistry models based on quantum consideration are under development [6] and recent work showed the capacity of such models to handle complex reactive air flows in place of the usual phenomenological models.

Finally, the lack of well-defined flight and ground-test measurements exploitable in the rarefied regime does not permit to demonstrate the accuracy of DSMC for reliable simulations of reentry flows. The carrying of experiments in rarefied and hypersonic conditions are essential for the acquisition of complementary aerodynamic and aerothermodynamic data as well as for the improvement of DSMC models. For that purpose, several requirements must be met:

- Using an advanced instrumentation made of sensors suited for such conditions

- Performing a thorough pre and post-experiment analysis of the object's wall in order to identify and quantify any catalytic, ablative and accomodation phenomenon

- Having access to a precise geometry of the tested object 
- Knowing precisely the freestream test conditions in order to alleviate the major sources of uncertainty

To this day, dealing with all those criteria can be challenging but the design of specific flight missions such as orbit transfers instead of classic reentries would be an ideal way of collecting such data while avoiding continuum conditions.

\section{Acknowledgment}

This work was supported by the ONERA and DGA (Direction Générale de l'Armement), the French Ministry of Armed Forces, through the co-funding of the $\mathrm{PhD}$ of M. Schouler. This work was also granted access to the HPC resources of CALMIP under the allocation 2020 - Project 20018. ONERA HPC resources have also been used.

\section{References}

[1] G. A. Bird, Molecular gas dynamics and the direct simulation of gas flows, Clarendon Press, 1994.

[2] I. D. Boyd, T. E. Schwartzentruber, Nonequilibrium gas dynamics and molecular simulation, Cambridge University Press, Cambridge, 2017. doi:10.1017/9781139683494.

[3] M. J. Wright, F. S. Milos, P. Tran, Afterbody aeroheating flight data for planetary probe thermal protection system design, Journal of Spacecraft and Rockets 43 (5) (2006) 929-943. doi:10.2514/1.17703.

[4] P. Reynier, Survey of aerodynamics and aerothermodynamics efforts carried out in the frame of Mars exploration projects, Progress in Aerospace Sciences 70 (2014) 1-27. doi:10.1016/j.paerosci.2014.03.004.

[5] B. R. Hollis, S. Borrelli, Aerothermodynamics of blunt body entry vehicles, Progress in Aerospace Sciences 48-49 (2012) 42-56. doi:10.1016/j.paerosci.2011.09.005.

[6] T. E. Schwartzentruber, I. D. Boyd, Progress and future prospects for particle-based simulation of hypersonic flow, Progress in Aerospace Sciences 72 (2015) 66-79. doi:10.1016/j.paerosci.2014.09.003.

[7] P. Reynier, Survey of high-enthalpy shock facilities in the perspective of radiation and chemical kinetics investigations, Progress in Aerospace Sciences 85 (2016) 1-32. doi:10.1016/j.paerosci.2016.04.002.

[8] J. Allègre, M. Raffin, A. Chpoun, L. Gottesdienert, Rarefied Hypersonic Flow over a Flat Plate with Truncated Leading Edge, American Institute of Aeronautics and Astronautics, Washington DC, 1994. doi:10.2514/5.9781600866326.0285.0295.

[9] J. F. Padilla, Comparison of DAC and MONACO DSMC Codes With Flat Plate Simulation, Tech. Rep. August, NASA (2010).

[10] C. White, M. K. Borg, T. J. Scanlon, S. M. Longshaw, B. John, D. R. Emerson, J. M. Reese, dsmcFoam+: An OpenFOAM based direct simulation Monte Carlo solver, Computer Physics Communications 224 (2018) 22-43. doi:10.1016/j.cpc.2017.09.030.

[11] R. C. Palharini, C. White, T. J. Scanlon, R. E. Brown, M. K. Borg, J. M. Reese, Benchmark numerical simulations of rarefied non-reacting gas flows using an open-source DSMC code, Computers and Fluids 120 (2015) 140-157. doi:10.1016/j.compfluid.2015.07.021.

[12] S. J. Plimpton, S. G. Moore, A. Borner, A. K. Stagg, T. P. Koehler, J. R. Torczynski, M. A. Gallis, Direct simulation Monte Carlo on petaflop supercomputers and beyond, Physics of Fluids 31 (8) (2019) 1-18. doi:10.1063/1.5108534.

[13] C. Borgnakke, P. S. Larsen, Statistical collision model for Monte Carlo simulation of polyatomic gas mixture, Journal of Computational Physics 18 (4) (1975) 405-420. doi:10.1016/0021-9991(75)90094-7.

[14] D. J. Rader, J. N. Castaneda, J. R. Torczynski, T. W. Grasser, W. M. Trott, Measurements of thermal accommodation coefficients., Tech. rep. (2005). doi:10.2172/876357.

[15] W. M. Trott, J. N. Castaeda, J. R. Torczynski, M. A. Gallis, D. J. Rader, An experimental assembly for precise measurement of thermal accommodation coefficients, Review of Scientific Instruments 82 (3) (2011) 1-12. doi:10.1063/1.3571269.

[16] G. J. LeBeau, F. E. Lumpkin, Application highlights of the DSMC analysis code (DAC) software for simulating rarefied flows, Computer Methods in Applied Mechanics and Engineering 191 (6-7) (2001) 595-609. doi:10.1016/S0045-7825(01)00304-8.

[17] S. Dietrich, I. D. Boyd, Scalar and parallel optimized implementation of the direct simulation Monte Carlo method, Journal of Computational Physics 126 (2) (1996) 328-342. doi:10.1006/jcph.1996.0141.

[18] K. S. Heffner, L. Gottesdiener, A. Chpoun, J. C. Lengrand, Leading edge effect on rarefied hypersonic flow over a flat plate, in: AIAA 22nd Fluid Dynamics, Plasma Dynamics and Lasers Conference, 1991, 1991. doi:10.2514/6.1991-1749. 
[19] J.-C. Lengrand, A. Chpoun, Rarefied Hypersonic Flow over a Sharp Flat Plate: Numerical and Experimental Results, no. November 2015, 1994. doi:10.2514/5.9781600866326.0276.0284.

[20] J. M. Burt, E. Josyula, I. D. Boyd, Novel Cartesian implementation of the direct simulation Monte Carlo method, Journal of Thermophysics and Heat Transfer 26 (2) (2012) 258-270. doi:10.2514/1.T3733.

[21] H. Yamaguchi, N. Tsuboi, Y. Matsumoto, 3D DSMC Simulation of rarefied hypersonic flow over a sharp flat plate, in: AIP Conference Proceedings, Vol. 585, 2001, pp. 764-771. doi:10.1063/1.1407636.

[22] J. F. Padilla, I. D. Boyd, Assessment of gas-surface interaction models in DSMC analysis of rarefied hypersonic flow, in: Collection of Technical Papers - 39th AIAA Thermophysics Conference, Vol. 1, 2007, pp. 1-15. doi:10.2514/6.2007-3891.

[23] J. F. Padilla, I. D. Boyd, Assessment of Gas-surface interaction models for computation of rarefied hypersonic flow, Journal of Thermophysics and Heat Transfer 23 (1) (2009) 96-105. doi:10.2514/1.36375.

[24] E. Cecil, J. McDaniel, Planar Velocity and Temperature Measurements in Rarefied Hypersonic Flow Using Iodine LIF, in: 38th AIAA Thermophysics Conference, American Institute of Aeronautics and Astronautics, Reston, Virigina, 2005. doi:10.2514/6.2005-4695.

[25] J. N. Moss, J.-C. Lengrand, Hypersonic Experimental and Computational Capability, Improvement and Validation, in: AGARD-AR-319 Vol. II, 1998, Ch. Rarefied F.

[26] J. Allègre, D. Bisch, J.-C. Lengrand, Experimental Rarefied Density Flowfields at Hypersonic Conditions over 70 Degree Blunted Cone, Journal of Spacecraft and Rockets 34 (6) (1997) 714-718.

[27] J. Allègre, D. Bisch, J.-C. Lengrand, Experimental Rarefied Heat Transfer at Hypersonic Conditions over 70 Degree Blunted Cone, Journal of Spacecraft and Rockets 34 (6) (1997) 724-728.

[28] J. Allègre, D. Bisch, J.-C. Lengrand, Experimental Rarefied Aerodynamic Forces at Hypersonic Conditions over 70 Degree Blunted Cone, Journal of Spacecraft and Rockets 34 (6) (1997) 719-723.

[29] A. Klothakis, I. K. Nikolos, Modeling of Rarefied Hypersonic Flows Using the Massively Parallel Dsmc Kernel " Sparta ", in: 8th International Congress on Computational Mechanics, Vol. 12, 2015.

[30] J. N. Moss, T. Pot, B. Chanetz, M. Lefebvre, DSMC Simulation of Shock / Shock Interactions : Emphasis on Type IV Interactions, Tech. rep. (1998).

[31] J. N. Moss, About Simulations Sharp Double of Shock Cones Interactions, Tech. Rep. August, NASA $(2000)$.

[32] J. N. Moss, G. A. Bird, G. N. Markelov, DSMC simulations of hypersonic flows and comparison with experiments, in: AIP Conference Proceedings, Vol. 762, 2005, pp. 547-552. doi:10.1063/1.1941593.

[33] J. Murphy, Flight Test Aerodynamic Heating Data for the Afterbody of the Project Mercury Spacecraft With Comparisons To Available Prediction Methods, Tech. rep., NASA (1967).

[34] D. R. Chapman, An Analysis of Base Pressure at Supersonic Velocities and Comparison with Experiment, Tech. rep., NASA (1951). doi:19930090963.

[35] L. Jacchia, Thermospheric Temperature, Density, and Composition: New Models, Tech. rep., NASA (1977).

[36] D. B. Lee, W. D. Goodrich, Apollo Experience Report - Aerothermodynamics Evaluation, Tech. rep., NASA (1972).

[37] J. E. Pavlosky, L. G. St Leger, Apollo experience report: Thermal protection subsystem, Tech. Rep. January 1974, NASA (1974).

[38] M. J. Wright, D. K. Prabhu, E. R. Martinez, Analysis of apollo command module afterbody heating part I: AS-202, Journal of Thermophysics and Heat Transfer 20 (1) (2006) 16-30. doi:10.2514/1.15873.

[39] L. M. Walpot, M. J. Wright, P. Noeding, F. Schrijer, Base flow investigation of the Apollo AS-202 Command Module, Progress in Aerospace Sciences 48-49 (2012) 57-74. doi:10.1016/j.paerosci.2011.06.006.

[40] J. N. Moss, C. E. Glass, F. A. Greene, DSMC simulations of apollo capsule aerodynamics for hypersonic rarefied conditions, in: 9th AIAA/ASME Joint Thermophysics and Heat Transfer Conference Proceedings, Vol. 3, 2006, pp. 1733-1750. doi:10.2514/6.2006-3577.

[41] J. N. Moss, C. Glass, F. Greene, Blunt body aerodynamics for hypersonic low density flows, in: 25th International Symposium on Rarefied Gas Dynamics, 2006, pp. 1-6.

[42] K. C. Tseng, J. S. Wu, I. D. Boyd, Simulations of Re-entry vehicles by using DSMC with chemical-reaction module, in: A Collection of Technical Papers - 14th AIAA/AHI International Space Planes and Hypersonic Systems and Technologies Conference, Vol. 3, 2006, pp. 1789-1801. doi:10.2514/6.2006-8084.

[43] M.-C. Lo, C.-C. Su, J.-S. Wu, K.-C. Tseng, Modelling Rarefied Hypersonic Reactive Flows Using the Direct Simulation Monte Carlo Method, Communications in Computational Physics 18 (4) (2015) 1095-1121. doi:10.4208/cicp.080115.010515s.

[44] J. F. Padilla, I. D. Boyd, Assessment of rarefied hypersonic aerodynamics modeling and windtunnel data, in: Collection of Technical Papers - 9th AIAA/ASME Joint Thermophysics and Heat Transfer Conference Proceedings, Vol. 2, 2006, pp. 1247-1262. doi:10.2514/6.2006-3390. 
[45] G. A. Bird, The DS2V/3V program suite for DSMC calculations, in: AIP Conference Proceedings, Vol. 762, 2005, pp. 541-546. doi:10.1063/1.1941592.

[46] R. C. Millikan, D. R. White, Systematics of vibrational relaxation, The Journal of Chemical Physics 39 (12) (1963) 3209-3213. doi:10.1063/1.1734182.

[47] C. Park, Review of chemical-kinetic problems of future NASA missions. I - Earth entries, Journal of Thermophysics and Heat Transfer 7 (3) (1993) 9-23. doi:10.2514/3.496.

[48] P. A. Gnoffo, R. N. Gupta, J. L. Shinn, Conservation equations and physical models for hypersonic air flows in thermal and chemical nonequilibrium, Tech. Rep. 2867, NASA (1989).

[49] P. A. Gnoffo, An upwind-biased, point-implicit relaxation algorithm for viscous, compressible perfectgas flows., Tech. rep., NASA (1990).

[50] Anon, U. S. Standard Atmosphere, 1962, Tech. rep., NASA (1962).

[51] T. Banyai, E. Torres, A. V. Kashkovsky, P. V. Vashchenkov, M. S. Ivanov, P. Rambaud, Direct Monte Carlo Simulations of the Intermediate Experimental Vehicle at Early Stages of Reentry, in: Proc. of 7th European Symposium on Aerothermodynamics, Brugge, 2011.

[52] D. B. Lee, W. D. Goodrich, The Aerothermodynamic Environment of the Apollo Command Module During Superorbital Entry, Tech. rep., NASA (1972).

[53] E. R. Hillje, Entry Aerodynamics at Lunar Return Conditions Obtained from the Flight of Apollo 4, Tech. rep., NASA (1969).

[54] D. B. Lee, J. J. Bertin, Heat-Transfer Rate and Pressure Measurements Obtained During Apollo Orbital Entries, Tech. rep., NASA (1970).

[55] E. R. Hillje, Entry Flight Aerodynamics from Apollo Mission AS-202, Tech. rep., NASA (1967).

[56] R. Gardon, A Transducer for the Measurement of Heat-Flow Rate, Journal of Heat Transfer (1960) $396-398$.

[57] K. Sutton, A. J. Graves, A general stagnation-point convective-heating equation for arbitrary gas mixtures, Tech. Rep. November 1971, NASA (1971).

[58] K. Sutton, Air Radiation Revisited, in: AIAA 19th Thermophysics Conference, Snowmass, 1984.

[59] C. O. Johnston, A. M. Brandis, Features of afterbody radiative heating for earth entry, Journal of Spacecraft and Rockets 52 (1) (2015) 105-119. doi:10.2514/1.A33084.

[60] D. Zimpfer, P. Hattis, J. Ruppert, D. Gavert, Space shuttle GN and C development history and evolution, in: AIAA SPACE Conference and Exposition 2011, no. April, 2011. doi:10.2514/6.20117244.

[61] A. M. Whitnah, E. R. Hillje, Space Shuttle Wind Tunnel Testing Program Summary, Tech. rep., NASA (1984). doi:10.2514/6.1982-562.

[62] J. C. Young, J. M. Underwood, E. R. Hillje, A. M. Whitnah, P. O. Romere, J. D. Gamble, B. B. Roberts, G. M. Ware, W. I. Scallion, B. Spencer, J. P. Arrington, D. C. Olsen, Aerodynamic Challenges of the Design and Development of the Space Shuttle Orbiter, in: NASA Conference Publication, no. pt 1, 1985, pp. 209-263.

[63] J. W. Haney, Orbiter Entry Heating Lessons Learned from Development Flight Test Program, in: NASA Conference Publication, no. pt 2, 1983, pp. 719-751.

[64] D. M. Curry, Space Shuttle Orbiter Thermal Protection System Design and Flight Experience, Tech. rep., NASA (1993).

[65] J. L. Shinn, J. N. Moss, A. Simmonds, Viscous-Shock-Layer Heating Analysis for the Shuttle Windward Plane with Surface Finite Catalytic Recombination Rates, in: 3rd Joint Thermophysics, Fluids, Plasma and Heat Transfer Conference, 1982.

[66] D. A. Throckmorton, Shuttle entry aerothermodynamic flight research: The orbiter experiments (OEX) program, in: AIAA 17th Aerospace Ground Testing Conference, Vol. 30, 1992, pp. 449-465. doi:10.2514/6.1992-3987.

[67] R. C. Blanchard, G. M. Buckt, Determination of rarefied-flow aerodynamics of the shuttle orbiter from flight measurements on STS-6 and STS-7, in: AlAA 23rd Aerospace Sciences Meeting, 1985, 1985. doi:10.2514/6.1985-347.

[68] R. C. Blanchard, J. F. Rutherford, Shuttle orbiter high resolution accelerometer package experiment: Preliminary flight results, Journal of Spacecraft and Rockets 22 (4) (1985) 474-480. doi:10.2514/3.25775.

[69] R. C. Blanchard, G. M. Buck, Rarefied-flow aerodynamics and thermosphere structure from shuttle flight measurements, Journal of Spacecraft and Rockets 23 (1) (1986) 18-24. doi:10.2514/3.25078.

[70] M. A. Gallis, K. A. Boyles, G. J. Lebeau, DSMC simulations in support of the STS-107 accident investigation, in: AIP Conference Proceedings, Vol. 762, 2005, pp. 1211-1216. doi:10.1063/1.1941698.

[71] J. N. Moss, G. A. Bird, Direct Simulation of Transitional Flow for Hypersonic Reentry Conditions, in: AIAA, 1988, pp. 830-843. doi:10.2514/2.6909.

[72] J. C. Adams, W. R. Martindale, A. W. Mayne, M. E. O., Real gas scale effects on hypersonic laminar 
boundary-layer parameters including effects of entropy-layer swallowing, in: AIAA 9th Fluid and Plasma Dynamics Conference, 1976, pp. 1-12. doi:https://doi.org/10.2514/6.1981-1042.

[73] E. V. Zoby, Approximate Heating Analysis for the Windward Symmetry Plane of Shuttle-Like Bodies At Large Angle of Attack., Progress in Astronautics and Aeronautics 82 (1982) 229-247.

[74] J. L. Shinn, A. L. Simmonds, Comparison of Viscous-Shock-Layer Heating Analysis With Shuttle Flight Data in Slip Flow Regime, in: AIAA, 1984, pp. 1-9. doi:10.2514/5.9781600865718.0491.0510.

[75] G. A. Bird, Application of the Direct Simulation Monte Carlo Method to the Full Shuttle Geometry, in: AIAA/ASME 5th Joint Thermophysics and Heat Transfer Conference, 1990.

[76] D. RAULT, Aerodynamics of Shuttle Orbiter at high altitudes, Journal of Spacecraft and Rockets 31 (6) (1994) 944-952. doi:10.2514/6.1993-2815.

[77] C. Park, S. Yoon, Calculation of real-gas effects on blunt-body trim angles, AIAA Journal 30 (4) (1992) 999-1007. doi:10.2514/3.11020.

[78] K. W. Iliff, M. F. Shafer, Space shuttle hypersonic aerodynamic and aerothermodynamic flight research and the comparison to ground test results, Tech. rep. (1993).

[79] Unknown, Aerodynamic Design Data Book - Vol. I: Orbiter Vehicle, JSC-19654, 1978.

[80] R. C. Blanchard, Rarefield Flow Lift-to-Drag Measurements of the Shuttle Orbiter, in: Congress of the International Council of the Aeronautical Sciences, Vol. 2, 1986, pp. 1421-1430.

[81] S. Matsumoto, Y. Kondoh, S. Nagai, R. Tagai, T. Imada, E. Nakano, Aerodynamic oscillation and attitude control analysis for reentry capsule using OREX flight data and wind tunnel data, in: AIAA Guidance, Navigation, and Control Conference, no. January, 2016, pp. 1-11. doi:10.2514/6.2016-1131.

[82] R. N. Gupta, J. N. Moss, J. M. Price, Assessment of Thermochemical Nonequilibrium and Slip Effects for Orbital Reentry Expriment (OREX), in: 31st AIAA Thermophysics Conference, New Orleans, 1996.

[83] J. N. Moss, R. N. Gupta, J. M. Price, DSMC Simulations of OREX Entry Conditions, Tech. rep., NASA (1996).

[84] J. N. Moss, G. A. Bird, Monte carlo simulations in support of the shuttle upper atmospheric mass spectrometer experiment, Journal of Thermophysics and Heat Transfer 2 (2) (1988) 138-144. doi:10.2514/3.77.

[85] R. Müller-Eigner, G. Koppenwallner, B. Fritsche, Pressure and Heat Flux Measurement with RAFLEX II During MIRKA Re-entry, in: Third European Symposium on Aerothermodynamics for Space Vehicles, Noordwijk, 1998.

[86] G. Herdrich, M. Fertig, D. Petkow, A. Steinbeck, Modeling approaches for gas-surface interactions, in: 48th AIAA Aerospace Sciences Meeting Including the New Horizons Forum and Aerospace Exposition, 2010. doi:10.2514/6.2010-1467.

[87] J. Pallegoix, J. Collinet, Atmospheric Re entry Demonstrator : Post Flight Analysis, Flight Rebuilding with CFD Control., in: 2nd International Symposium Atmospheric Reentry Vehicles and Systems, no. March, Arcachon, 2001.

[88] M. Fertig, H.-H. Frühauf, Detailed Computation of the Aerothermodynamic Loads of the Mirka Capsule, in: Aerothermodynamics for space vehicles, Proceedings of the 3rd European Symposium on Aerothermodynamics for space vehicles held at ESTEC, Noordwijk, The Netherlands, 24-26 November 1998, 1999, pp. 703-710.

[89] G. A. Bird, Nonequilibrium radiation during re-entry at $10 \mathrm{~km} / \mathrm{s}$, in: AIAA 22nd Thermophysics Conference, Honolulu, 1987. doi:10.2514/6.1987-1543.

[90] M. Wright, J. Brown, K. Sinha, G. Candler, Validation of Afterbody Aeroheating Predictions for Planetary Probes: Status and Future Work, in: 2nd International Planetary Probe Workshop, no. 1, 2005, pp. 275-286.

[91] M. Wright, M. Loomis, P. Papadopoulos, Aerothermal analysis of the project fire II afterbody flow, Journal of Thermophysics and Heat Transfer 17 (2) (2003) 240-249. doi:10.2514/2.6757.

[92] G. E. Woodbury, Angle-of-Attack analysis for Project Fire 1 Payload Reentry Flight, Tech. Rep. April, NASA (1966).

[93] J. N. Moss, G. A. Bird, V. Dogra, Nonequilibrium thermal radiation for an aeroassist flight experiment vehicle, in: 26th Aerospace Sciences Meeting, 1988. doi:10.2514/6.1988-81.

[94] J. C. Taylor, A. B. Carlson, H. A. Hassan, Monte Carlo Simulation of Reentry Flows With Ionization, in: 30th Aerospace and Sciences Meeting and Exhibit, 1992.

[95] J. C. Taylor, A. B. Carlson, H. A. Hassan, Monte Carlo simulation of radiating reentry flows, in: AIAA 28th Thermophysics Conference, Vol. 8, 1993, pp. 478-485. doi:10.2514/6.1993-2809.

[96] E. D. Farbar, I. D. Boyd, Simulation of FIRE II reentry flow using the direct simulation Monte Carlo method, in: 40th AIAA Thermophysics Conference, 2008. doi:10.2514/6.2008-4103.

[97] D. S. Liechty, C. O. Johnston, M. J. Lewis, Comparison of DSMC and CFD solutions of fire II including radiative heating, in: 42nd AIAA Thermophysics Conference, 2011, pp. 1-14. doi:10.2514/6.2011-3494. 
[98] D. S. Liechty, M. J. Lewis, Extension of a kinetic-theory approach for computing chemical-reaction rates to reactions with charged particles, in: AIP Conference Proceedings, Vol. 1333, 2011, pp. 12391244. doi:10.1063/1.3562813.

[99] C. O. Johnston, B. R. Hollis, K. Sutton, Non-boltzmann modeling for air shock-layer radiation at lunar-return conditions, Journal of Spacecraft and Rockets 45 (5) (2008) 879-890. doi:10.2514/1.33006.

[100] C. O. Johnston, B. R. Hollis, K. Sutton, Nonequilibrium stagnation-line radiative heating for Fire II, Journal of Spacecraft and Rockets 45 (6) (2008) 1185-1195. doi:10.2514/1.33008.

[101] M. Panesi, T. Magin, A. Bourdon, A. Bultel, O. Chazot, Fire II flight experiment analysis by means of a collisional-radiative model, Journal of Thermophysics and Heat Transfer 23 (2) (2009) 236-248. doi:10.2514/1.39034.

[102] W. L. J. Jones, A. E. Cross, Electrostatic-Probe Measurments of Plasma Parameters for Reentry Flight Experiments at 25000 Feet Per Second, Tech. rep., NASA (1972).

[103] G. A. Bird, Computation of electron density in high altitude re-entry flows, in: AIAA 20th Fluid Dynamics, Plasma Dynamics and Lasers Conference, 1989. doi:10.2514/6.1989-1882.

[104] I. D. Boyd, Modeling of associative ionization reactions in hypersonic rarefied flows, Physics of Fluids 19 (9). doi:10.1063/1.2771662.

[105] M. Fang, Z. Li, Z. Li, C. Li, DSMC Approach for Rarefied Air Ionization during Spacecraft Reentry 1 Introduction, Communications in Computational Physics 23 (4) (2018) 1167-1190. doi:10.4208/cicp.OA-2016-0186.

[106] Y. Liu, D. Prabhu, K. A. Trumble, D. Saunders, P. Jenniskens, Radiation modeling for the reentry of the stardust sample return capsule, Journal of Spacecraft and Rockets 47 (5) (2010) 741-752. doi:10.2514/1.37813.

[107] D. A. Kontinos, M. Stackpoole, Post-flight analysis of the stardust sample return capsule Earth Entry, in: 46th AIAA Aerospace Sciences Meeting and Exhibit, no. January, 2008. doi:10.2514/6.2008-1201.

[108] R. A. Micheltree, R. G. Wilmoth, F. M. Cheatwood, G. J. Brauckmann, F. A. Green, Aerodynamics of Stardust Sample Return Capsule, AIAA, 1997.

[109] R. G. Wilmoth, R. A. Mitcheltree, J. N. Moss, Low-density aerodynamics of the Stardust sample return capsule, Journal of Spacecraft and Rockets 36 (3) (1999) 436-441. doi:10.2514/2.3464.

[110] T. Ozawa, Z. Li, I. Sohn, D. A. Levin, M. F. Modest, Modeling of electronic excitation and radiation for hypersonic reentry flows in DSMC, in: 48th AIAA Aerospace Sciences Meeting Including the New Horizons Forum and Aerospace Exposition, no. January, 2010, pp. 4-7. doi:10.2514/6.2010-987.

[111] J. Zhong, T. Ozawa, D. A. Levin, Modeling of stardust reentry ablation flows in the near-continuum flight regime, AIAA Journal 46 (10) (2008) 2568-2581. doi:10.2514/1.36196.

[112] P. Jenniskens, Observations of the stardust sample return capsule entry with a slitless echelle spectrograph, Journal of Spacecraft and Rockets 47 (5) (2010) 718-735. doi:10.2514/1.37518.

[113] I. D. Boyd, K. A. Trumble, M. J. Wright, Modeling of stardust entry at high altitude, part 1: Flowfield analysis, Journal of Spacecraft and Rockets 47 (5) (2010) 708-717. doi:10.2514/1.37360.

[114] I. D. Boyd, P. M. Jenniskens, Modeling of stardust entry at high altitude, part 2: Radiation analysis, Journal of Spacecraft and Rockets 47 (6) (2010) 901-909. doi:10.2514/1.37357.

[115] T. K. Mankodi, U. V. Bhandarkar, B. P. Puranik, Hypersonic flow over Stardust Re-entry Capsule using ab-initio based chemical reaction model, Acta Astronautica 162 (January) (2019) 243-255. doi:10.1016/j.actaastro.2019.06.021.

[116] S. F. Gimelshein, I. J. Wysong, Bird's total collision energy model: 4 decades and going strong, Physics of Fluids 31 (7) (2019) 1-16. doi:10.1063/1.5097706. 